\author{
UNIVERSIDADE DE SÃO PAULO \\ FFCLRP - DEPARTAMENTO DE FÍSICA \\ PÓS-GRADUAÇÃO EM FÍSICA APLICADA À MEDICINA E BIOLOGIA
}

\title{
Nanopartículas de magnetita dopadas com zinco para fins teranósticos: magnetoacustografia e hipertermia magnética
}

\author{
YASER HADADIAN
}

Tese apresentada à Faculdade de Filosofia, Ciências e Letras de Ribeirão Preto da Universidade de São Paulo, como parte das exigências para a obtenção do título de Doutor em Ciências, Área: Física aplicada à Medicina e Biologia.

Ribeirão Preto - SP 


\section{Nanopartículas de magnetita dopadas com zinco para fins teranósticos: magnetoacustografia e hipertermia magnética}

Tese apresentada à Faculdade de Filosofia, Ciências e Letras de Ribeirão Preto da Universidade de São Paulo, como parte das exigências para a obtenção do título de Doutor em Ciências.

Área de Concentração:

Física aplicada à Medicina e Biologia.

Orientador:

Theo Zeferino Pavan

Coorientador:

Ana Paula Ramos

Ribeirão Preto -SP 


\title{
Zinc substituted magnetite nanoparticles for theranostic purpose: magnetomotive ultrasound imaging and magnetic hyperthermia
}

\begin{abstract}
A thesis submitted in partial fulfillment of the requirements for the degree of Doctor of Philosophy Department of Physics, Faculty of Philosophy, Sciences and literature of Ribeirão Preto, University of São Paulo.
\end{abstract}

Area:

Physics Applied to Medicine and Biology

Supervisor:

Theo Zeferino Pavan

Co-supervisor:

Ana Paula Ramos

Ribeirão Preto -SP 
Autorizo a reprodução e divulgação total ou parcial deste trabalho, por qualquer meio convencional ou eletrônico, para fins de estudo e pesquisa, desde que citada a fonte.

\section{FICHA CATALOGRÁFICA}

Hadadian, Yaser

Nanopartículas de magnetita dopadas com zinco para fins teranósticos: magnetoacustografia e hipertermia magnética / Yaser Hadadian; orientador Theo Zeferino Pavan. Ribeirão Preto - SP, 2019.

111 f.:il.

Tese (Doutorado - Programa de Pós-Graduação em Física Aplicada à Medicina e Biologia) - Faculdade de Filosofia, Ciências e Letras de Ribeirão Preto da Universidade de São Paulo, 2019.

1. Nanopartículas magnéticas. 2. Magnetoacustografia 3. Hipertermia magnética. 4. Ultrassom 
Nome: Yaser Hadadian

Título: Nanopartículas de magnetita dopadas com zinco para fins teranósticos: magnetoacustografia e hipertermia magnética

Title: Zinc substituted magnetite nanoparticles for theranostic purpose: magnetomotive ultrasound imaging and magnetic hyperthermia

Tese apresentada à Faculdade de Filosofia, Ciências e Letras de Ribeirão Preto da Universidade de São Paulo, como parte das exigências para a obtenção do título de Doutor em Ciências.

Aprovado em:

Banca examinadora

Prof. Dr.: Instituição:

Julgamento: Assinatura:

Prof. Dr.: Instituição:

Julgamento: Assinatura:

Prof. Dr.: Instituição:

Julgamento: Assinatura:

Prof. Dr.: Instituição:

Julgamento: Assinatura:

Prof. Dr.: Instituição:

Julgamento: Assinatura: 
To my parents and my sisters for all their love and support 


\section{Acknowledgements}

First and foremost, I would like to express my deepest gratitude to my supervisor Prof. Dr. Theo Zeferino Pavan who was always present and patient to discuss my work and to help me. In addition to providing me his precious support and knowledge, his encouragements and allowing me the research freedom helped me to gain invaluable experiences during my $\mathrm{PhD}$. I am also very grateful of my co-supervisor Prof. Dr. Ana Paula Ramos for all her supports in chemistry laboratory.

I would like to express my sincere gratitude to Professor Antonio Adilton Oliveira Carneiro for all his supports in research and outside research during my $\mathrm{PhD}$.

I am also grateful for the help, assistance and friendship of the past and current group members and all technicians in physics department, in particular, Elcio Aparecido Navas, Agnelo dos Santos Bastos, Carlos Renato Silva.

Finally, tons of thanks are extended to my close friends, especially Mehran, for their encouragement and support.

This work was mainly supported by Coordenação de Aperfeiçoamento de Pessoal de Nível Superior (CAPES) - Finance Code 001, which I am very grateful of their support. I should also thank Conselho Nacional de Desenvolvimento Científico e Tecnológico (CNPq) and Fundação de Amparo à Pesquisa do Estado de São Paulo (FAPESP) for their financial support in this study. 


\section{Resumo}

Hadadian, Yaser. Nanopartículas de magnetita dopadas com zinco para fins teranósticos: magnetoacustografia e hipertermia magnética. 2019 127f. Tese (Doutorado - Programa de Pós-graduação em Física Aplicada à Medicina e Biologia) - Faculdade de Filosofia, Ciências e Letras de Ribeirão Preto, Universidade de São Paulo, Ribeirão Preto - SP 2019

A nanociência e a nanotecnologia abriram o caminho para novos e promissores avanços em diferentes campos da ciência, incluindo a medicina. Por exemplo, nanopartículas magnéticas têm sido amplamente utilizadas como agentes teranósticos. Neste contexto, elas podem ser usadas para terapia em hipertermia magnética e como agente de contraste em imagens de magnetoacustografia (MMUS). Esta tese apresenta o resultado de um estudo sistemático sobre o potencial teranóstico de nanopartículas de magnetita dopadas com zinco $\left(\mathrm{Zn}_{\mathrm{x}} \mathrm{Fe}_{1-\mathrm{x}} \mathrm{Fe}_{2} \mathrm{O}_{4}, \mathrm{x}=\right.$ $0,1,0,2,0,3,0,4)$. Usando um método de coprecipitação simples e econômico, o $\mathrm{Fe}^{2+}$ foi substituído por $\mathrm{Zn}^{2+}$ na estrutura de magnetita para otimizar suas propriedades magnéticas. Um aumento significativo na saturação magnética para a amostra $\mathrm{x}=0,1$ foi alcançado, resultando em um aumento de mais de duas vezes no deslocamento induzido pela força magnética em imagens MMUS em comparação com a magnetita pura. O desempenho das nanopartículas preparadas foi investigado para hipertermia magnética sob uma ampla gama de condições experimentais. Neste caso, a amostra $\mathrm{x}=0,1$ também apresentou uma eficiência de aquecimento superior em relação às demais. Além disso, integramos imagens de MMUS e térmica por ultrassom com hipertermia magnética em uma única plataforma. Essa plataforma teranóstica foi capaz de aplicar campos magnéticos para gerar imagens MMUS e para o procedimento de hipertermia usando uma única bobina. Durante o procedimento de hipertermia, mapas de temperatura bidimensional foram adquiridos em tempo real. O sistema foi testado com sucesso utilizando a amostra $\mathrm{Zn}_{0,1} \mathrm{Fe}_{0,9} \mathrm{Fe}_{2} \mathrm{O}_{4}$. Embora essa plataforma tenha sido avaliada em um experimento com simuladores de tecido, que está longe de ser realista, ela pode abrir um novo horizonte para o planejamento da hipertermia magnética, devido à sua simplicidade, baixo custo, ser não invasiva e ter potencial para gerar imagens em tempo real. Finalmente, o projeto e a construção de um sistema de hipertermia magnética também são apresentados nesta tese.

Palavras-chave: Nanopartículas magnéticas; Magnetoacustografia; Hipertermia magnética; Ultrassom 


\begin{abstract}
Hadadian, Yaser. Zinc substituted magnetite nanoparticles for theranostic purpose: magnetomotive ultrasound imaging and magnetic hyperthermia. 2019. 127p. A thesis submitted in partial fulfillment of the requirements for the degree of Doctor of Philosophy Department of Physics, Faculty of Philosophy, Sciences and literature of Ribeirão Preto - SP, 2019.
\end{abstract}

Nanoscience and nontechnology have paved the way for new and promising advances in different scientific disciplines including medicine. For example, magnetic nanoparticles have been extensively used as theranostic agents. In this context, they can be used for therapy in magnetic hyperthermia and as contrast agent in magnetomotive ultrasound (MMUS) imaging. This thesis presents the result of a systematic study on the theranostic potential of zinc substituted magnetite nanoparticles $\left(\mathrm{Zn}_{\mathrm{x}} \mathrm{Fe}_{1-\mathrm{x}} \mathrm{Fe}_{2} \mathrm{O}_{4}, \mathrm{x}=0.1,0.2,0.3,0.4\right)$. Using a simple and cost effective coprecipitation route, $\mathrm{Zn}^{2+}$ was substituted for $\mathrm{Fe}^{2+}$ in magnetite structure to optimize its magnetic properties. A significant increase in saturation magnetization at zinc content $\mathrm{x}=0.1$ was achieved, resulting in more than twofold increase in induced displacement for MMUS compared to the pure magnetite. The performance of the prepared nanoparticles was investigated for magnetic hyperthermia under a wide range of experimental conditions. In this case, also, sample $\mathrm{x}=0.1$ showed a superior heating efficiency compared to the others. In addition, we integrated magnetomotive and thermal ultrasound imaging with magnetic hyperthermia in a single platform. This theranostic platform was capable of acquiring MMUS images, applying a radiofrequency magnetic field for the hyperthermia procedure, and providing a real-time two-dimensional temperature map. The system was successfully tested using the sample $\mathrm{Zn}_{0.1} \mathrm{Fe}_{0.9} \mathrm{Fe}_{2} \mathrm{O}_{4}$, offering an entire theranostic platform in the field of magnetic hyperthermia. Although this platform was evaluated in a phantom experiment, which is far from realistic conditions, it may open a new horizon for magnetic hyperthermia planning, owing to its simplicity, low-cost, real-time, and non-invasive features. Finally, the design and construction of a cross-coupled inverter for the magnetic hyperthermia system is also presented in this thesis.

Keywords: Magnetic nanoparticles; Magnetomotive ultrasound imaging; Magnetic hyperthermia; Ultrasound 


\section{Table of Figures}

Figure 1.1 a) Schematics of a prolate nanoparticle and b) a plot of magnetic anisotropy as a

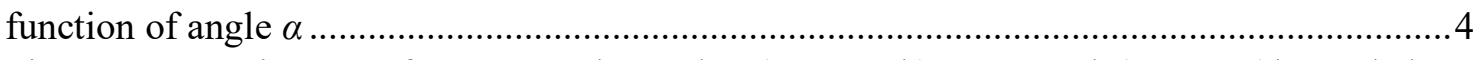
Figure 2.1TEM images of as-prepared samples a) $\mathrm{x}=0.0, \mathrm{~b}$ ) $\mathrm{x}=0.1$ and $\mathrm{c}$ ) $\mathrm{x}=0.4$ (the scale bar is the same for all images).

Figure 2.2 Variation of the average particle size with zinc content obtained from TEM

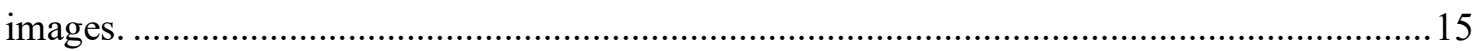

Figure 2.3 X-ray diffraction patterns of synthesized Zn-substituted magnetite nanoparticles.

Diffraction peaks are labeled based on the JCPDS 19-0629 standard card. ........................... 17

Figure 2.4 Calculated crystallite sizes for different zinc content in magnetite structure.........17

Figure 2.5 The effect of zinc substitution on lattice parameter of magnetite, the inset shows the shit for peaks of (511) and (440) planes to the lower diffraction angles. .......................... 18

Figure 2.6 FTIR spectra of prepared magnetite nanoparticles............................................ 19

Figure 2.7 (a) Room temperature M-H curves of the samples, (b) the effect of zinc substitution on saturation magnetization of as-prepared samples.

Figure 2.8 Zero-field-cooling (ZFC) measurements of the samples by plotting the imaginary

component of the AC susceptibility with respect to temperature..... 22 Figure 2.9 A typical extrapolation of curves for the variation of magnetization with respect to temperature (a) and observed Curie temperature of the samples (b) ..................................23 Figure 2.10 The variation of magnetization with respect to temperature for sample with $x=0$ (magnetite).

Figure 3.1 Schematic depiction of the experimental setup for MMUS experiments. A low frequency magnetic field is applied to the phantom containing an inclusion with magnetic nanoparticles and the induced microvibrations is detected using ultrasound imaging............30 Figure 3.2 A typical B-mode image of the phantoms with magnetic nanoparticle $(x=0.1)$ inclusion (a) and its MMUS image (b).

Figure 3.3 Observed displacement in the inclusions for each phantom prepared with different magnetic nanoparticles (a) and magnetization values at applied magnetic field of $0.1 \mathrm{~T}$ for different zinc concentration in magnetite structure (b).

Figure 3.4 The observed displacement in gelatin phantoms for the phantoms containing inclusions filled with the nanoparticles prepared with different amounts of zinc.

Figure 3.5 A typical power spectrum of the oscillation frequency resulting from the interaction of magnetic nanoparticles with the applied field

Figure 4.1 (a) A conventional Mazzilli circuit, (b) the block diagram of the proposed system.

Figure 4.2 The complete diagram of the proposed circuit. In this circuit, A and B are the active gate drives.

Figure 4.3 (a) Pictures of the designed coils, (b) the setup for the in vitro experiment with cell culture plates using the HL coil, (c) and the setup for gelatin phantom experiments using the P coil.

Figure 4.4 Voltage signal across (a) Q1 and Q2, (b) the gate of Q1 and drain of Q2, (c) and the resonant coil with its power spectrum.

Figure 4.5 Simulated and measured on-axis magnetic field profiles of (a) S1 and (b) S2 coils, (c) measured on-axis and (d) 3D representation of the experimental magnetic field of HL coil. 
Figure 4.6 (a) Measured and (b) simulated z-component of the magnetic field of $\mathrm{P}$ coil. Measured and simulated magnetic fields of the P coil: (c) x or y-axis $1 \mathrm{~mm}$ away from its surface, and (d) z-axis.

Figure 4.7 Temperature rise at different frequencies with a field amplitude of $3.1 \mathrm{kA} / \mathrm{m}$ using S1 coil, (b) temperature rise at maximum field strength of HL, S1, S2, and P coils using 1200 $\mathrm{nF}$ capacitance for the tank circuit, and (c) the determined SAR values.

Figure 5.1 The measured SLP values of the samples at $H_{0}=7.5 \mathrm{kA} / \mathrm{m}$ and different concentrations for (a) $339 \mathrm{kHz}$, (b) $240 \mathrm{kHz}$, and (c) $137 \mathrm{kHz}$. d) Mean ILP values considering all frequencies.

Figure 5.2 SLP field dependence (a) for sample $\mathrm{x}=0.2,0.3$ and 0.4 and (b) for sample $\mathrm{x}=0.0$ and 0.1 for $c=1.5 \mathrm{wt} . \%$. (c) SLP field dependence for sample $\mathrm{x}=0.1$ and $c=3.5,1.5$, and 0.3 wt.\%. d) SLP frequency dependence for all samples at $c=2.5 \mathrm{wt} . \%$.

Figure 5.3 The dynamic hysteresis loops of the samples considering $f=137 \mathrm{kHz}$ and $H_{0}=7.5$

$\mathrm{kA} / \mathrm{m}$.

Figure 5.4 (a) - (e) SLP variation versus $\sigma=K_{\text {eff }} V / k_{B} T$ considering the volumes obtained from TEM images (the dashed lines represent the experimental values), and (f) the calculated and experimental values of SLP for all samples for $f=339 \mathrm{kHz}$ and at $c=0.3 \%$.

Figure 5.5 Simulated SLP versus (a) $\sigma$ and frequency for $H_{0}=7.5 \mathrm{kA} / \mathrm{m}$, and (b) field amplitude and frequency for $\sigma=6.7$.

Figure 5.6 Dipolar coupling constant $(\lambda)$ and (b) relative strength of the dipolar interaction at different concentrations.

Figure 5.7 Energy conversion efficiency versus frequency for all samples.

Figure 6.1 A schematic depiction of the proposed theranostic platform. The pancake coil was driven by two different power supplies to generate a pulse (for MMUS) and a radiofrequency magnetic field (for magnetic hyperthermia). In both procedures, a clinical ultrasound device was employed to acquire the MMUS and thermal images.

Figure 6.2 a) Magnetic field strength on the central axis of the coil, $2 \mathrm{~mm}$ away from its surface. b) Induced displacements measured in the center of the inclusion. Figure 6.3 a) B-mode image of the phantom acquired using unfocused plane waves emission. b) MMUS peak displacement image; c) and d) show images of the time to reach the peak displacements for $\mathrm{t}<3$ and $\mathrm{t}<6.5 \mathrm{~ms}$, respectively

Figure 6.4 The final MMUS image overlaid on the B-mode image.

Figure 6.5 a) B-mode image obtained using the SonixRP clinical imaging software, b) an example of the apparent displacement map, and c) its thermal strain map at the field amplitude $7.5 \mathrm{kA} / \mathrm{m}$.

Figure 6.6 Thermal images obtained 14, 28, $66 \mathrm{~s}$ after starting the hyperthermia procedure using the magnetic field strength of $9.2 \mathrm{kA} / \mathrm{m}$.

Figure 6.7 Comparison between the temperature measurement using the fiber optic thermometer and ultrasound thermal imaging.

Figure 6.8 Correlation between the temperature rise (heating rate) and the observed displacement independent of the spatial position. 


\section{List of Tables}

Table 2.1 EXD analysis of nominal compositions $\mathrm{Zn}_{\mathrm{x}} \mathrm{Fe}_{1-\mathrm{x}} \mathrm{Fe}_{2} \mathrm{O}_{4}, \mathrm{x}=0.0,0.1,0.2,0.3,0.4 \ldots 16$

Table 4.1 The physical characteristics of the designed coils

Table 4.2 The measured frequencies $(\mathrm{kHz})$ and maximum field strength $(\mathrm{kA} / \mathrm{m})$ for different coils at five capacitance configurations.

Table 5.1 Characteristics of the samples, the numbers in parentheses for $\langle d\rangle$ are the polydispersity degree calculated as (standard deviation/mean particle diameter) and for $D_{\mathrm{h}}$ are the polydispersity index (PDI) provided by the zeta-sizer instrument. 


\section{Table of Content}

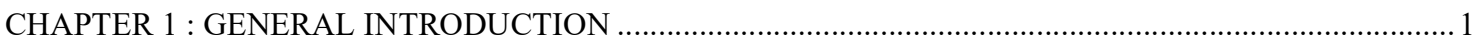

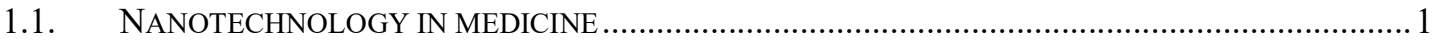

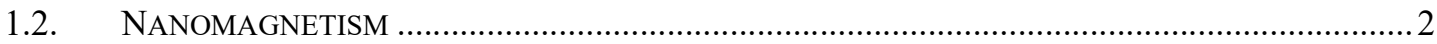

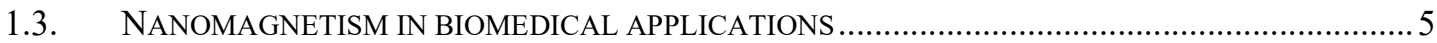

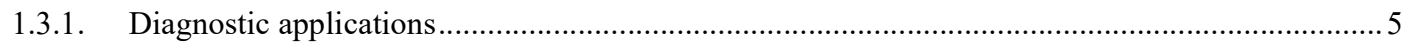

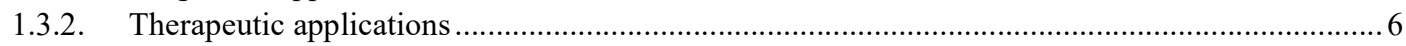

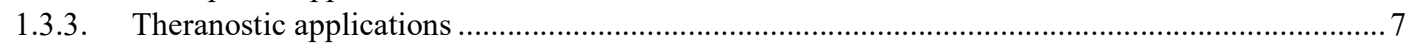

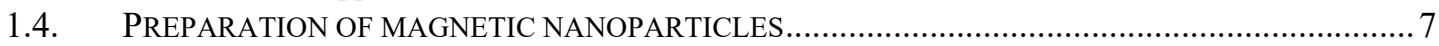

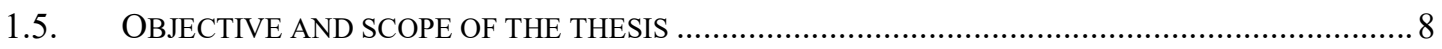

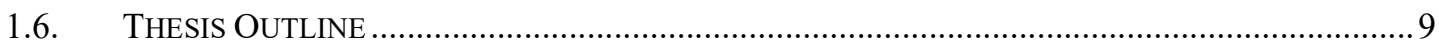

CHAPTER 2 : SYNTHESIS AND CHARACTERIZATION OF ZINC SUBSTITUTED MAGNETITE

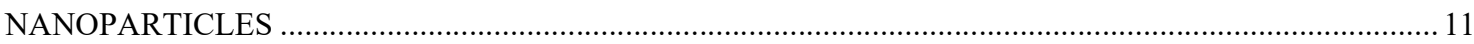

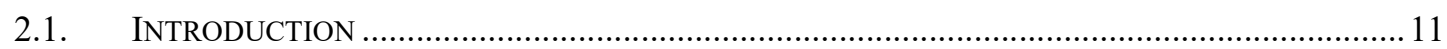

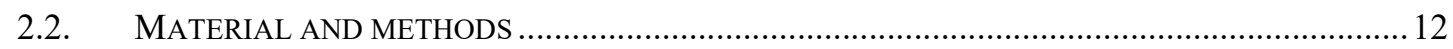

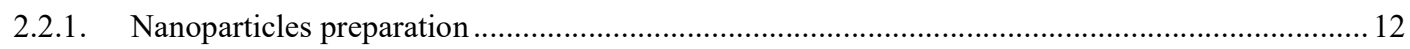

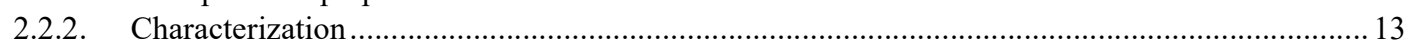

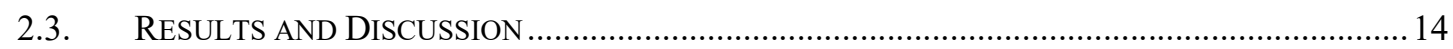

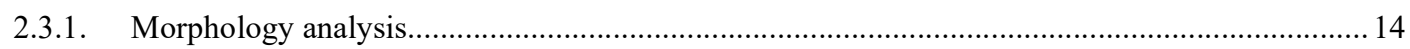

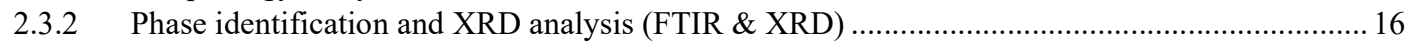

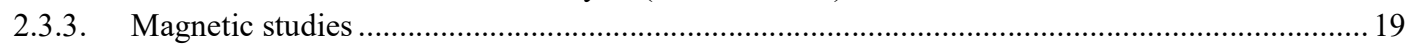

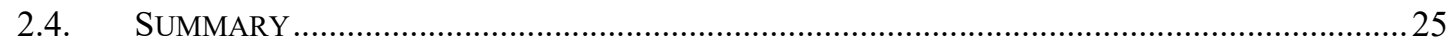

CHAPTER 3 : MAGNETOMOTIVE ULTRASOUND IMAGING USING ZINC SUBSTITUTED

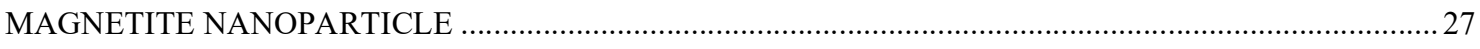

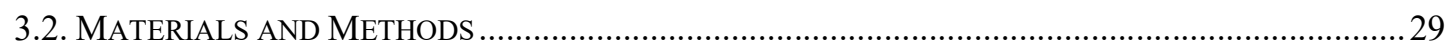

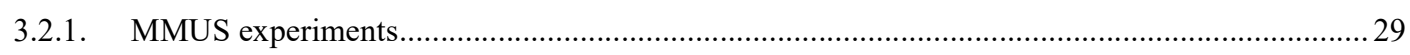

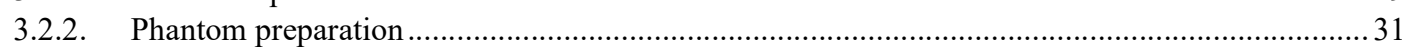

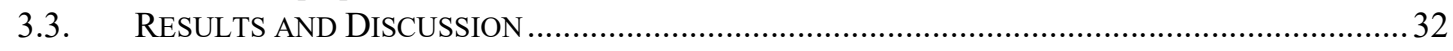

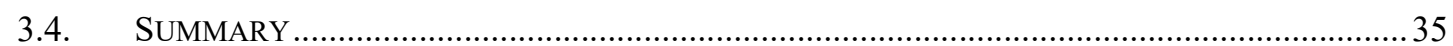

CHAPTER 4 : A VERSATILE INDUCTION HEATING SYSTEM FOR MAGNETIC HYPERTHERMIA STUDIES UNDER DIFFERENT EXPERIMENTAL CONDITIONS …………………................................. 36

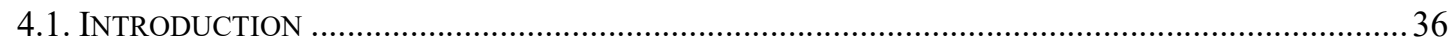

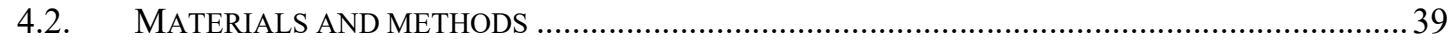

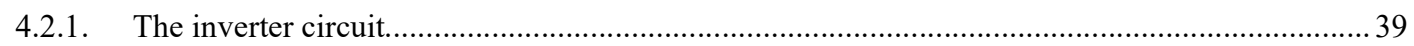

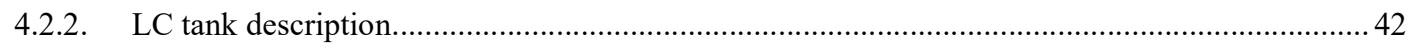

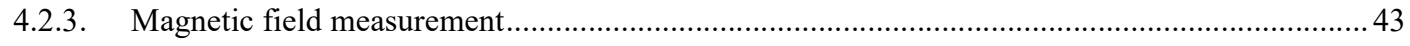

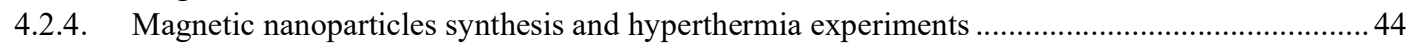

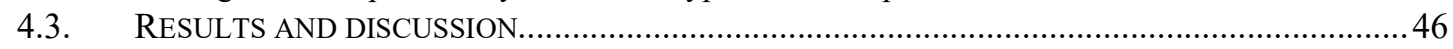

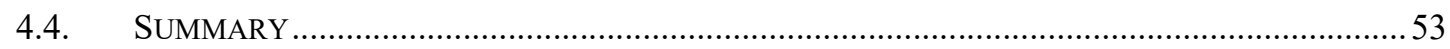

CHAPTER 5 : ROLE OF ZINC SUBSTITUTION IN MAGNETIC HYPERTHERMIA PROPERTIES OF MAGNETITE NANOPARTICLES: INTERPLAY BETWEEN INTRINSIC PROPERTIES AND DIPOLAR

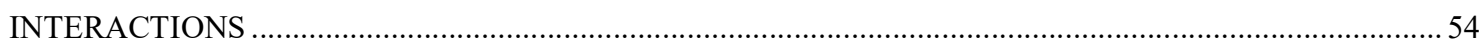

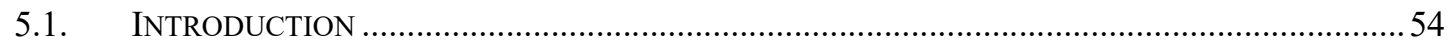

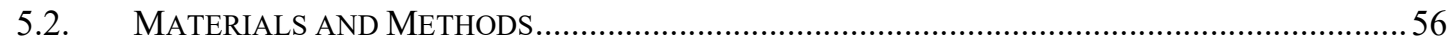

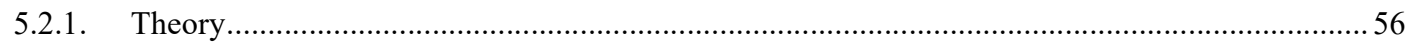

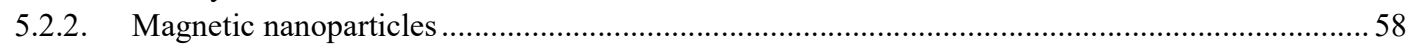

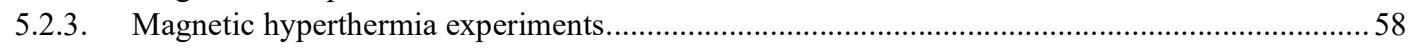

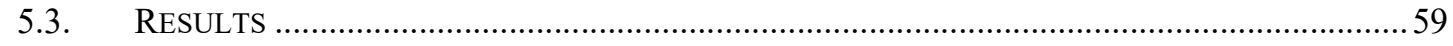

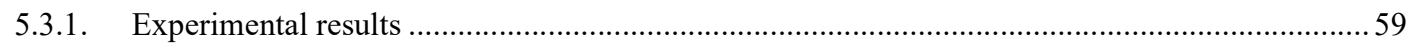




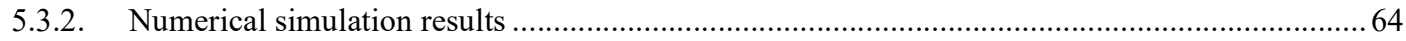

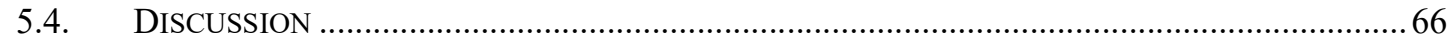

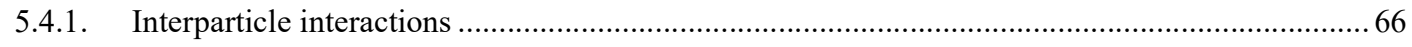

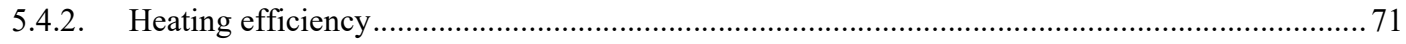

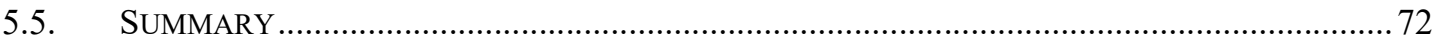

CHAPTER 6 : A NOVEL THERANOSTIC PLATFORM: INTEGRATION OF MAGNETOMOTIVE AND THERMAL ULTRASOUND IMAGING WITH MAGNETIC HYPERTHERMIA ........................................ 74

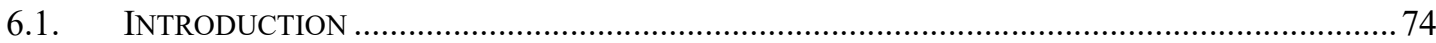

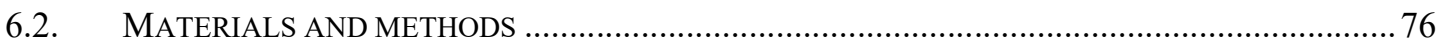

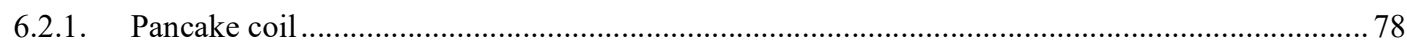

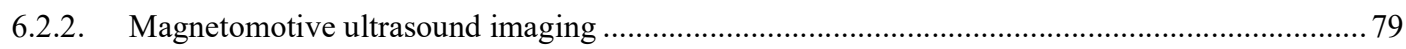

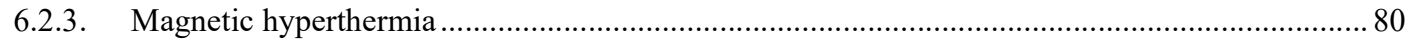

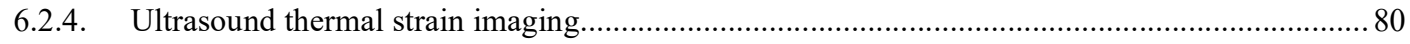

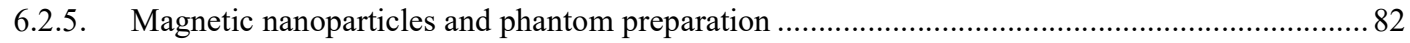

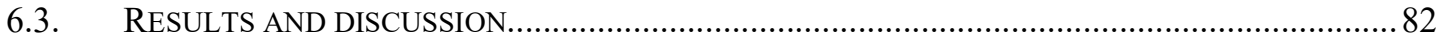

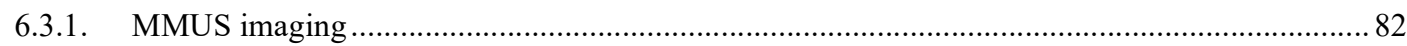

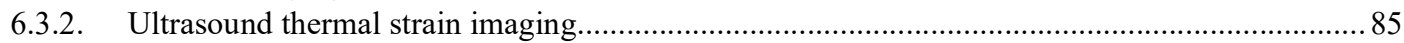

6.3.3. Comparing temperature elevation during hyperthermia and induced displacements during MMUS 89

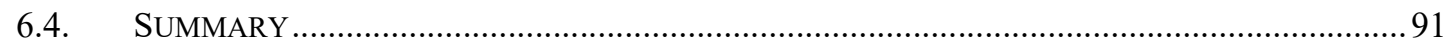

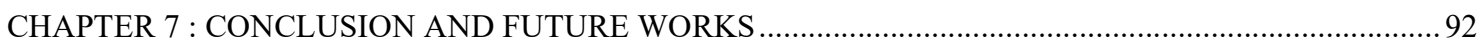

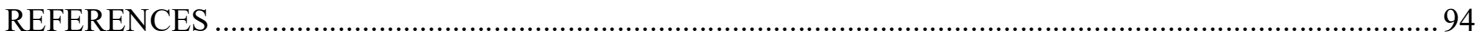




\section{Chapter 1 : General Introduction}

Cancer is characterized by out-of-control growth and spreading of abnormal cells [1]. Despite the remarkable advances in understanding the molecular biology, chemotherapy, radiotherapy and conventional surgical procedures, the morbidity and mortality associated with cancer are increasing globally. It is projected that the annual number of cancer incident rises from 18.1 in 2018 to 29.4 million in 2040 [2]. According to the World Health Organization [3], this increase will be higher in low-income countries (more than 80\%) compared to high-income countries $(40 \%)$. Cancer is the first or second leading cause of death in the population under 70 years old in more than $50 \%$ of countries. These together with other facts and statistics on cancer $[2,4]$ show the huge health and economic burden that cancer can have worldwide. Therefore, research on developing new techniques for early stage diagnosis and effective therapy approaches with minimal side effects among medical professionals and scientists has attracted extensive attention.

\subsection{Nanotechnology in medicine}

In recent decades, nanoscience and nanotechnology have paved the way for remarkable advancements in various areas such as electronics, magnetism, communication, environmental, life, and medical science. In particular, the convergence of nanotechnology, medicine and biology has developed promising research areas referred to as nanomedicine and nanobiotechnology $[5,6]$. This has led to a breakthrough in the diagnosis and therapy of several diseases, including cancers [7]. In cancer research, especially, nanomedicine is significantly involved since it can provide new diagnostic and therapeutic approaches to overcome some drawbacks of conventional treatments such as the development of resistance to chemo- and radiotherapy, poor solubility of hydrophobic anti-cancer drugs, side effects on healthy tissues, usage of harmful radiation etc. [8]. Moreover, nanotechnology may facilitate the targeted delivery of drugs to a specific cell or tissue, transcytosis of drugs across tight epithelial and endothelial barriers, delivery of the large macromolecules to intercellular sites, simultaneous delivery of two or more drugs, monitoring the drug delivery using combined therapeutic and imaging agents etc. [9].

In biomedical application, among different nanomaterials, magnetic nanoparticles have always been in the center of attention due to their unique intrinsic properties, which enables them to 
be used in a wide range of application. They may be used as contrast agents in several imaging techniques [10] for locating and diagnosing cancerous cells, carriers for targeted drug delivery [11], pattern recognition [12], magnetic separation [13], biosensor [14] and in therapy routines [1]. This class of nanomaterials include metallic, bimetallic, and ferrite based compositions [15]. However, ferrite nanoparticles, particularly iron oxides, have been widely favored due to their interesting magnetic properties and their biocompatibility [16, 17]. The possibility of tuning the intrinsic properties of ferrite nanoparticles by changing their composition, size, and shape through their synthesis provides enormous opportunities for their application in various areas.

\subsection{Nanomagnetism}

Magnetic materials and their behavior when immersed in a magnetic field may be classified and characterized based on their magnetic susceptibility, which describes the relation between the applied magnetic field and the magnetization induced in the material due to the field. For small and negative susceptibility values, the material is considered as diamagnetic, and small but positive values give rise to para- and antiferromagnetic behaviour. Ferro- and ferrimagnetic materials are known by their large and positive susceptibility, which is a function of the applied magnetic field [18]. Although paramagnetism, diamagnetism, and antiferromagnetism play an important role when studying the atomic and molecular structure of materials, these effects are very weak when it comes to many practical applications. Pronounced magnetic effects, occurs only in few metallic elements such as $\mathrm{Fe}, \mathrm{Co}, \mathrm{Ni}, \mathrm{Mn}$, etc. and some of the rare earths. Alloys and oxides of these elements are constituent of ferro- or ferrimagnetic substances [19].

The spontaneous magnetization, due to the alignment of the magnetic moments, is the characteristic feature of a ferromagnet. The cooperative interaction of a great number of atomic spins in ferromagnetic materials creates small regions where all atomic spins are aligned parallel (positive exchange interaction) [19]. These regions, known as magnetic domain, are spontaneously magnetized, however, in the absence of an external magnetic field they are arranged such that the net magnetization of the material may be zero. However, application of a relatively small field changes the domains arrangement, and hence leads to an appreciable net magnetization. In antiferromagnetic materials, on the other hand, there exist a negative exchange interaction, resulting in antiparallel alignment of neighboring spins and no net spontaneous magnetization. This is understood by considering two sublattices in the atomic structure of the materials with equal magnitude of magnetic moment, hence, zero spontaneous 
magnetization. Ferrimagnets exhibit properties which can be similar to both ferro- and antiferromagnets. In this case, the presence of two different sublattices is analogous to antiferromagnets but the different values of their magnetic moments result in a spontaneous magnetization $[18,20,21]$.

Magnetic domains are basically formed to reduce the total magnetostatic energy of the system. The process of splitting the material into smaller regions continues up to a certain point where the energy cost of adding more domain walls (the boundary separating domains) is greater than the magnetostatic energy saved [22]. This procedure determines a critical size beyond which it will be energetically more favorable for the specimen to divide itself into two or more domains. In other words, as size decreases to a value smaller than this critical size, the particle will be consisted of only one domain (single domain state), wherein all the magnetic moments are aligned parallel. This critical size varies depending on the material and for a spherical particle can be determined as $[23,24]$ :

$$
D_{c r} \approx \frac{72 \sqrt{A K}}{\mu_{0} M_{s}^{2}}
$$

where $A$ is the exchange stiffness parameter, $K$ is the uniaxial anisotropy constant, $M_{\mathrm{s}}$ is the saturation magnetization, and $\mu_{0}$ the vacuum magnetic permeability.

While there are various definition for nanosize materials, they are mostly know as structures having at least one dimension ranging between 1 to $100 \mathrm{~nm}$ [25]. This is the range where the size of nanoparticles is comparable to their magnetic domain and magnetic properties are size dependent to a large extent. In fact, many novel phenomena and magnetic effects occur in nanosize magnetic materials. In addition, in nanoscale the large surface to volume ratio, where the number of surface atoms becomes a considerable fraction of the total number of atoms and broken symmetry at the boundaries happen, several important consequences to the physical properties of the system may be observed. Of particular interest, in magnetic materials, is the superparamagnetism [26] regime which happens in single domain magnetic nanoparticles. Many biomedical applications of magnetic nanoparticles are determined according to their superparamagnetic properties [15].

Typically, a single domain nanoparticle with uniaxial anisotropy is modeled by a prolate spheroid (figure 1.1(a)) with easy axis of magnetization along the major axis. Then, the magnetic anisotropy energy of the sample is described as [15]: 


$$
E_{A}=K V \sin ^{2} \alpha
$$

where $\alpha$ is the angle between the direction of the magnetization $M$ and the easy axis, $K$ is the uniaxial magnetic anisotropy constant, and $V$ the volume of the particle. The magnetic anisotropy energy has two equal minima separated by an energy barrier $(K V)$. This can be visualized in figure 1.1(b) which is a plot of magnetic anisotropy as a function of angle $\alpha$. If the energy barrier is of the order of thermal energy $\left(k_{\mathrm{B}} T\right)$, the spontaneous magnetization reversal activated by thermal energy may happen on the timescale of the experiment and the specimen is said to be in superparamagnetism regime [15, 24, 27]. Applying a magnetic field with a random direction respect to the easy axis of the particle $(\theta)$ results in modifying these minima by lifting the energy degeneracy of the wells and decreasing the energy barrier [15].

Due to the dependence of the magnetization reversal on temperature, a characteristic crossover temperature, at where the magnetic moments appear "blocked", exists. At this temperature, known as blocking temperature, the timescale of the magnetization reversals matches that of the measurement. As a consequence, at a given temperature if the timescale of the magnetization measurement is larger than the relaxation time of the specimen, an S-shape closed loop appears, while for smaller timescales, an open hysteresis loop can be observed. Therefore, blocking temperature is not an intrinsic temperature and it depends on the measurement time [24]. However, for static magnetic measurements, particles with blocking temperature below room temperature exhibit superparamagnetic property.

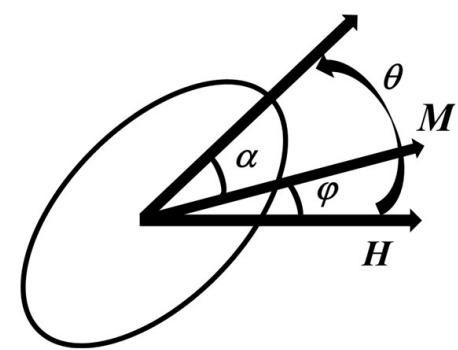

(a)

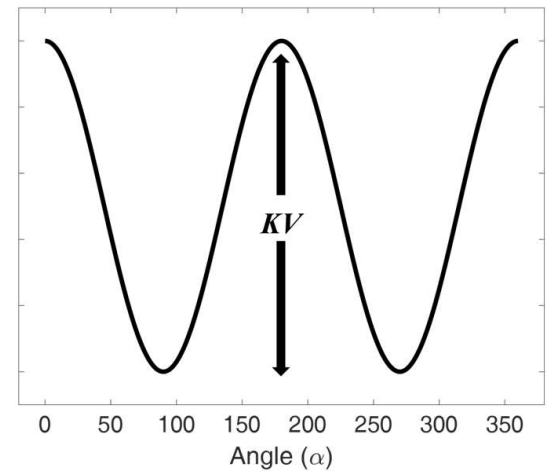

(b)

Figure 1.1 a) Schematics of a prolate nanoparticle and b) a plot of magnetic anisotropy as a function of angle $\alpha$

In order to obtain blocking temperature, usually zero field-cooled (ZFC) and field-cooled (FC) magnetization curves are employed. ZFC magnetization curves are typically taken by cooling 
the sample in zero field from higher temperatures where the sample exhibits superparamagnetic behaviour. Then, at the presence of a small applied field magnetization is measured while increasing the temperature. FC measurement can subsequently be recorded in the same applied magnetic field upon cooling the sample. The peak observed in ZFC measurements is considered as blocking temperature; however, one should keep in mind that it is not an absolute and characteristic temperature and depends strongly on the timescale of the measurement [24].

\subsection{Nanomagnetism in biomedical applications}

\subsubsection{Diagnostic applications}

Magnetic nanoparticles have been studied in several areas such as catalyzes [28], ferrofluids [29], high-density information magnetic storage devices [30]. Biomedical applications of magnetic nanoparticles in diagnostic and therapeutic routines are of particular interest in this thesis. For example, due to their intrinsic magnetic properties which is very different from biological systems, they have been used as source of labels in biosensors to recognize specific molecular targets [31]. Dynamic magnetization of nanoparticles can also be used for temperature estimation [32] and monitoring the viscosity and rigidity [33, 34]. Magnetic separation, water treatment, and food analysis are other applications of magnetic nanoparticles where magnetic gradient is employed for separation [35, 36].

One of the most frequent diagnostic application of magnetic nanoparticles is to be served as contrast agents in magnetic resonance imaging (MRI) [37, 38]. Magnetic nanoparticles can significantly modify the two independent relaxation processes, i.e. longitudinal and transverse relaxation processes, of their surrounding medium which are used to generate MR images. Local variation of the magnetic field due to the presence of nanoparticles, which arise from the large difference between the magnetic susceptibility of the magnetic nanoparticles and the tissues, result in corresponding image contrast. In addition, magnetic nanoparticles have been used as contrast agent in magnetomotive optical coherence tomography where the induced vibration to the nanoparticles by an external magnetic field is coupled to the highly-scattering surrounding tissue and detected as a periodic optical phase shift [39, 40]. Photoacoustic imaging is another modality wherein magnetic nanoparticles can efficiently be used as contrast agent for reducing the effect of background noises and generating high-contrast molecular images while in the same time they can be considerably more stable against laser irradiation compared to conventional gold nanoparticles $[41,42]$. 
Magnetic particle imaging [43] is another imaging modality which employs magnetic nanoparticles. In this case, basically, a time-variant applied field magnetizes the nanoparticles and their nonlinear magnetization generates a higher-order harmonic response. This timevariant magnetization induces a time-variant voltage inductively coupled pickup coil to generate the images. Finally, magnetomotive ultrasound (MMUS) imaging [44] is a recent radiation-free modality to acquire molecular images [45], obtain viscoelastic properties [46, 47], and also to localize magnetic nanoparticles within tissues [48]. Ultrasound is noninvasive, cost effective, portable, and deep penetrating; therefore, being the most widely used medical imaging modality; however, relatively low contrast has limited it to be considered as a molecular imaging technique. In addition, spatial resolution in ultrasound is not sufficient to directly visualize magnetic nanoparticles within tissues. MMUS imaging as a part of the present thesis will be discussed in more details in chapter 3.

\subsubsection{Therapeutic applications}

Drug delivery using a gradient magnetic field [49] have been investigated in clinical trials; however, it is limited to target tissues close to the body's surface [50]. A more efficient alternative for targeted drug delivery is to functionalize the magnetic nanoparticles by conjugating targeting ligands that bind to cell receptors highly expressed in tumor cells [51, 52]. In addition to drug delivery and controlled drug release [53], magnetic hyperthermia [54] is another therapeutic application. In magnetic hyperthermia, the released heat, due to the magnetization reversal process induced to the magnetic nanoparticles in the presence of a radiofrequency magnetic field, is employed to selectively induce apoptosis and/or necrosis in cancerous cells. Since the seminal work of Gilchrist [55] describing the use of ferrimagnetic particles to treat cancer, there have been tremendous effort [56] devoted to understanding the physical mechanism underlying this method, enhancing the intrinsic properties of the heating mediators, and improving the treatment procedure through different strategies such as targeted delivery of nanoparticles [57]. However, despite substantial promising results in preclinical practices, magnetic hyperthermia has not been considered as a standard technique for cancer treatment worldwide [58].

Enhancing the heat efficiency of nanoparticles has always been one of the main challenges in magnetic hyperthermia for translation from preclinical studies to clinical routines. This has been explored extensively by examining different materials or introducing several synthesis routes to tune the intrinsic properties of nanoparticles while keeping in mind the prerequisite 
of biocompatibility. Due this biocompatibility issue, the only nanomaterials that have been used in clinical studies are iron oxides (magnetite and maghemite) nanoparticles. They have also been used as contrast agents in different imaging modalities for cell labeling and molecular imaging, magnetic targeting for drug and gene delivery, cell separation etc.; or combination of diagnostic and therapeutic application as theranostic agents [59, 60]. However, many studies have shown that other ferrite based compositions can also offer high biocompatibility and excellent magnetic properties [37, 61-66]. In addition, accurate delivery of the nanoparticles to the target tissue, monitoring the temperature during the treatment, apparatus for generating adequate magnetic field for volumes as large as human body parts size, and safety limitation of applied magnetic field are other difficulties in clinical magnetic hyperthermia.

\subsubsection{Theranostic applications}

Theranostics is a relatively new term describing, in general, methods in which diagnosis and therapy are achieved through a single procedure or drug [67]. In cancer treatment, this approach is mainly carried out using nano agents capable of providing diagnostic imaging, delivering the desired therapy, and finally monitoring the response to the therapy [68]. This is the unique feature of nanotheranostic agents which provides the possibility of being imaged before, after, and even during the treatment. Moreover, these nanomaterials can be functionalized to be used to targeted delivery of anticancer therapeutics and/or as contrast agents for imaging [52, 6976]. In addition, utilizing nanotheranostic agents simultaneously with medical theranostic instruments [77-79] has recently been considered as an effective way to enhance the cancer treatment efficacy. However, despite substantial progresses in development of nanotheranostic agents, only few works has been reported on the development of theranostic instruments.

\subsection{Preparation of magnetic nanoparticles}

Since the most commonly used magnetic nanoparticles for biomedical applications are ferrite based materials, a brief review about few common synthesis methods will be presented here. Each method can present some advantages or disadvantages which determines its usefulness for each specific application.

Coprecipitation is one of the simplest and efficient method to synthesize magnetic nanoparticles (ferrites) [59, 80], which is performed using metal salts such as chlorides, sulfates, or nitrates dissolved in aqueous (acidic) solution that are "co-precipitated" upon the addition of a strong base such as $\mathrm{NH}_{4} \mathrm{OH}$ or $\mathrm{NaOH}$ at room temperature or at elevated temperatures. Size and shape of the resultant depends on many parameters such as the type of 
salts used, the salts ratio, the reaction temperature, the $\mathrm{pH}$ value, dropping speed of basic solution, ionic strength of the media, stirring rate [80]. Although there are studies reporting the synthesis of monodispersed nanoparticles by coprecipitation method [81], the major disadvantage of this method is poor control over the size distribution of the particles. However, to obtain a narrower size distribution in a post-synthesis process, some size sorting strategies such as size-grading procedure based on the addition of an electrolyte can be used [82, 83].

Microemulsion procedures have long been considered as a method to prepare magnetic nanoparticles with controlled size. This method can be divided into two groups, namely normal (oil-in-water) and reverse (water-in-oil) micelle methods [84]. It is a surfactant-based approach and the intrinsic properties of the resultant strongly depend on the type of the surfactant and the water-to-surfactant molar ratio [85]. The main disadvantages of this method is low yield, poor crystallinity and adverse effects of the residual surfactants on the magnetic properties of the particles $[86,87]$.

High quality and monodispersed magnetic nanoparticles can be obtained by thermal decomposition of organometallic compounds at high temperatures in a refluxing organic solvent and in the presence of surfactant [88]. Size and morphology of the particles can be tuned though reaction temperature and/or time, surfactant type and concentration. The resultant particles are usually hydrophobic and dispersed in organic solvent, which demands a further step to make them dispersible in water. Although thermal composition is considered as the gold standard method to synthesize particles with controlled size and morphology, it is one of the most complicated procedures, it involves laborious purification steps, is high cost and production is made at high temperatures $[80,85]$.

Hydrothermal synthesis is another method to prepare ferrite based nanoparticles which have two main routes; hydrolysis and oxidation or neutralization of mixed metal hydroxides [59]. This method is performed in aqueous solution at high temperatures ( 130 to $\left.250{ }^{\circ} \mathrm{C}\right)$ and a high vapor pressure from (0.3 to $4 \mathrm{MPa})$ [89] using a sealed autoclave. Although this method is one of the most efficient methods to obtain highly crystalline and monodisperse magnetic nanoparticles, it still fails to produce particles smaller than $10 \mathrm{~nm}$ with hydrophilic surface properties [87].

\subsection{Objective and scope of the thesis}

In this $\mathrm{PhD}$ thesis we aimed to synthesize ferrite based magnetic nanoparticles and evaluate their potential to be used as theranostic agents for cancer therapy. More specifically, this thesis 
is devoted to investigate the possibility of combining a diagnostic procedure (MMUS imaging) with a therapeutic one (magnetic hyperthermia) in a single nanoparticle composition. As it will be discussed in more detail in the following chapters, an ideal magnetic nanoparticle for MMUS imaging would be a superparamagnetic material possessing a high saturation magnetization/susceptibility (a soft magnetic material), while for magnetic hyperthermia there has to be a trade-off between different parameters including saturation magnetization, effective magnetic anisotropy, and size to achieve an acceptable heating efficiency. Due to high biocompatibility of magnetite nanoparticles, we planned to optimize the intrinsic properties of this composition through substitution of zinc in its structure. It was expected that in a composition with low zinc content an optimized magnetic characteristic would be achieve such that the resultant nanoparticles have the potential to be served as a theranostic agent.

Additionally, design and construction of a magnetic hyperthermia system is a part of this thesis, as well as novel theranostic platform by integrating MMUS and ultrasound thermal imaging with magnetic hyperthermia. This integrated system can have important implications to the field of magnetic hyperthermia, as it is the first time that a real-time, noninvasive and deep penetrating temperature maps is obtain during the procedure.

\subsection{Thesis Outline}

This thesis basically consists of research which have been published/submitted as four original articles and a proceeding paper published in an international conference. The thesis is organized as follow:

Chapter 2 presents the synthesis and characterization of zinc-substituted magnetic nanoparticles. This work [90] was published in the Journal of Magnetism and Magnetic Materials (JMMM).

Chapter 3 presents the results of the MMUS imaging using the prepared nanoparticles. This includes a part of the paper [90] published in JMMM and the proceeding paper [91] in the 2017 IEEE International Ultrasonics Symposium (IUS).

Chapter 4 describes the design, construction, and characterization of the magnetic hyperthermia system used here. This work [92] was published in Review of Scientific Instruments journal.

Chapter 5 presents the magnetic hyperthermia study of the prepared nanoparticles. This study deals with the effect of intrinsic magnetic properties and dipolar interactions on magnetic 
hyperthermia properties of the nanoparticles. It [93] has been published in Scientific Reports journal.

Chapter 6 describes a theranostic platform wherein MMUS imaging is used as diagnostic modality and ultrasound thermometry to map the temperature distribution during the hyperthermia procedure. The sample with composition $\mathrm{Zn}_{0.1} \mathrm{Fe}_{0.9} \mathrm{Fe}_{2} \mathrm{O}_{4}$ which showed superior performance in both MMUS and magnetic hyperthermia was used to evaluate the platform. This study has been accepted in IEEE Transactions on Biomedical Engineering journal [94].

Chapter 7 is a general conclusion of this thesis and suggestions for future works. 


\section{Chapter 2 : Synthesis and characterization of zinc substituted magnetite nanoparticles}

\subsection{Introduction}

Nanoscience and nontechnology in recent decades have paved the way for new and promising advances in different scientific disciplines. Nanomagnetism has received much attention from researchers in different areas such as high-density information magnetic storage devices [30], catalyzes [28], ferrofluids [29], water treatment [36], environmental technology [95]. In addition nanomagnetism has had a significant impact in medicine and biology, ranging from DNA and cell separation [36, 96] and biosensors [97] to revolutionizing diagnostic and therapeutic techniques $[98,99]$. However, functionalizing and tuning the magnetic properties of different magnetic nanomaterials for each application, considering some factors such as being cost-effective or biocompatible in medical application, is yet critical.

Magnetic oxides with ferrimagnetic properties and iron as the main metallic component are referred as ferrites. Ferrites with spinel cubic structure have attracted much attention due to great potential in different technological application. The general formula of spinel ferrites can be written as $\left[\mathrm{M}_{1-\mathrm{i}} \mathrm{Fe}_{\mathrm{i}}\right]^{\mathrm{A}}\left[\mathrm{M}_{\mathrm{i}} \mathrm{Fe}_{(2-\mathrm{i})}\right]^{\mathrm{B}} \mathrm{O}_{4}$, where $\mathrm{M}$ is a divalent cation such as $\mathrm{Fe}^{2+}, \mathrm{Mn}^{2+}, \mathrm{Ni}^{2+}$, $\mathrm{Zn}^{2+}, \mathrm{Co}^{2+}$.The spinel structure is formed by a face centered cubic (fcc) lattice of 32 oxygen. There are 96 interstices, 64 tetrahedral and 32 octahedral sites (A and B in the general formula), between the oxygen atoms where only 24 of them are occupied by cations. Of the 64 tetrahedral interstices, 8 are occupied and the remaining 16 cations occupy half of the 32 octahedral sites [100]. In the general formula of spinel structure, $i$ is the inversion parameter; for $i=0$ the structure is known as normal spinel and for $\mathrm{i}=1$ it is inverse spinel. However, in addition to these two extremes, a complete range of intermediate cation distribution is possible and this distribution may change with temperature and synthesis method [101]. Peculiar magnetic properties of ferrites strictly depend not only on the type of cations in their structure but also on the distribution over tetrahedral and octahedral sites in spinel structure. This can provide a wide variety of magnetic properties for ferrites which in turn offers a high potential to be used in different applications. 
Magnetite, $\mathrm{Fe}_{3} \mathrm{O}_{4}$, has an inverse spinel structure in bulk form at room temperature and can be written as $\left[\mathrm{Fe}^{3+}\right]^{\mathrm{A}}\left[\mathrm{Fe}^{2+} \mathrm{Fe}^{3+}\right]^{\mathrm{B}} \mathrm{O}_{4}[102]$. Magnetite nanoparticles can be designed as a highly biocompatible material [103] and have been the subject of many biomedical applications. In addition to being a biocompatible material, the magnetic properties of magnetite can be tuned for wide range of applications [104]. This magnetic tuning can be achieved either by changing the preparation method and conditions $[105,106]$ or by substituting different cations in magnetite structure. Saturation magnetization, Curie temperature and coercivity are examples of tunable magnetic properties that have been explored by many researches [37, 102, 107, 108].

The wide range of applications of magnetite has led to a great expansion in the methods of its preparation. Among different methods, coprecipitation is a common route of magnetic nanoparticle preparation for medical applications [109]. Coprecipitation process to prepare water-dispersible magnetic nanoparticles is cost-effective, fast, eco-friendly, reproducible, not complex, can be easily scaled up, can be done in low temperatures and without any toxic intermediate and solvents. In this method, simply by changing the synthesis condition, such as feed solution concentration, type of iron salts, mixing method, $\mathrm{pH}$, reaction temperature, calcination times and drying temperature, etc. the properties of the samples may change considerably [105]. These remarkable flexibility and simplicity of coprecipitation method have always made it a suitable and commonly used method for synthesis of iron oxide nanoparticles. This method also can present a few drawbacks such as particles aggregation, difficulty in controlling the size and its distribution.

It is well known that nanosize zinc ferrite has an anomalous magnetic behavior compared to its bulk counterpart [110] and the presences of zinc in other ferrites such as nickel, cobalt or magnetite plays an important role to optimize their magnetic properties $[37,111,112]$. In the present study, a cost-effective and simple coprecipitation method to manufacture $\mathrm{Zn}$ substituted magnetite nanoparticles $\left(\mathrm{Zn}_{\mathrm{x}} \mathrm{Fe}_{1-\mathrm{x}} \mathrm{Fe}_{2} \mathrm{O}_{4}, \mathrm{x}=0,0.1,0.2,0.3\right.$ and 0.4$)$ is reported and morphology, crystal structure, and magnetic properties of the particles are studied.

\subsection{Material and methods}

\subsubsection{Nanoparticles preparation}

Chemicals, including ferric chloride $\left(\mathrm{FeCl}_{3}\right)$, ferrous chloride tetrahydrate $\left(\mathrm{FeCl}_{2} \cdot 4 \mathrm{H}_{2} \mathrm{O}\right)$, zinc chloride $\left(\mathrm{ZnCl}_{2}\right)$, ammonium hydroxide solution were all purchased from Labsynth ${ }^{\circledR}$ company. All the reactants were used as received without any more purification. Deionized water from a Milli- $\mathrm{Q}^{\circledR}$ system was used throughout the procedures. 
All samples were synthesized at $25^{\circ} \mathrm{C}$ by one-pot coprecipitation method without any heat treatment. This method is based on the formation of aqueous solutions of chlorides, nitrates or sulphates of $\mathrm{Fe}^{3+}$, and/or $\mathrm{Fe}^{2+}$ and their simultaneous precipitation in the form of oxides, using bases such as $\mathrm{NaOH}$ or $\mathrm{NH}_{4} \mathrm{OH}$. The precipitate is then filtered, washed and dried. Depending on preparation conditions particles with a narrow size distribution in the range of 10 to $500 \mathrm{~nm}$ may be obtained, with a high purity [113].

To prepare $\mathrm{Zn}$-substituted magnetite $\left(\mathrm{Zn}_{\mathrm{x}} \mathrm{Fe}_{1-\mathrm{x}} \mathrm{Fe}_{2} \mathrm{O}_{4}, \quad \mathrm{x}=0.0,0.1, \quad 0.2, \quad 0.3\right.$ and 0.4$)$ nanoparticles, regarding the $\mathrm{x}$ values, stoichiometric solutions of the zinc and the iron salts were prepared separately. After obtaining a clear solution of each salt including $40 \mathrm{~mL}$ of $1 \mathrm{~mol} / \mathrm{L} \mathrm{FeCl}_{3}$ and $20 \mathrm{~mL}$ of $1 \mathrm{~mol} / \mathrm{L}(1-\mathrm{x}) \mathrm{FeCl}_{2} \cdot 4 \mathrm{H}_{2} \mathrm{O}+\mathrm{xZnCl}_{2}$ were all mixed together. Then, a $50 \mathrm{~mL}$ of $4 \mathrm{~mol} / \mathrm{L} \mathrm{NH}_{4} \mathrm{OH}$ aqueous solution was added to the mixture abruptly under vigorous mechanical stirring. Immediately after addition of the base a black precipitate (except for $\mathrm{x}=0.4$ which was dark brown) was formed and the stirring was maintained for 5 more minutes. Then, the final products were separated from the slurry by magnetic decanting and washed several times with deionized water in order to remove the excess of base and unwanted ions. Finally, the resulting precipitate was dried at room temperature (for 24 hours or over a day).

\subsubsection{Characterization}

Transmission electron microscopy (TEM) was used to observe the morphologies of the products. TEM images were taken using a Jeol JEM- 100 CXII instrument, equipped with Hamamatsu ORCA-HR digital camera. A drop of the colloidal dispersion in ethanol was deposited onto a copper grid covered with a conductive polymer and the solvent was then allowed to evaporate. The products were characterized by energy-dispersive X-ray spectroscopy (EDX) using an IXRF Systems, Inc., 500 Digital Processing. To confirm the formation of the desired phase, powder X-ray diffraction (XRD) patterns were recorded at room temperate using a BRUKER SIEMENS AXS D5005 X-Ray diffractometer with $\mathrm{Cu} \mathrm{K \alpha}$ radiation $(\lambda=1.5406 \AA$ ). To determine the peak positions, profile fitting and refinements were performed on the all X-ray patterns using Pseudo-Voigt function in PANalytical X'pert Highscore $^{\circledR}$ software. For further confirmation of the magnetite phase formation, Fourier transform infrared spectrum (FTIR) of the sample with $\mathrm{x}=0.0$ was acquired between 4000 and $400 \mathrm{~cm}^{-1}$ using a Bomem MB 100 spectrometer.

To study the magnetic properties of the samples, a vibrating sample magnetometer (VSM), QUANTUM DESIGN MPMS3® SQUID VSM DC magnetometer with the maximum external 
field of $50 \mathrm{kOe}$, was used. Magnetic behavior of all samples was investigated in three different conditions (or regimes); at room, low and high temperatures. At room temperature, field dependent magnetizations ( $\mathrm{M}-\mathrm{H}$ curves) were recorded to investigate the saturation magnetization of the samples. Blocking temperatures of the samples $\left(\mathrm{T}_{\mathrm{B}}\right)$ were determined by zero-field-cooling (ZFC) measurements from $5 \mathrm{~K}$ up to room temperature by applying a magnetic field of $100 \mathrm{Oe}$ with $1 \mathrm{kHz}$ frequency and recording the imaginary component of the $\mathrm{AC}$ susceptibility. To determine the Curie temperature, magnetization-temperature curves of the samples were recorded from room temperature up to $850 \mathrm{~K}$ by applying a static $100 \mathrm{Oe}$ magnetic field.

\subsection{Results and Discussion}

\subsubsection{Morphology analysis}

TEM analysis of as-synthesized samples revealed all nanoparticles were in the size range of 5 to $14 \mathrm{~nm}$ with reasonably narrow size distributions (figures 2.1 (a) and (b)). Particle size distributions and the average sizes were determined by measuring the diameter of at least 100 particles on each TEM image, using ImageJ software. Figure 2.2 shows the variation of the average particle size obtained from TEM images with the zinc content. As can be seen zinc substitution on the magnetite structure has led to decrease in particle sizes. As it has been already pointed out by Navrotsky [114], zinc obstructs the formation of spinel ferrite crystal. Crystal growth depends on different parameters, being the most important the molecular concentration of the starting reactants that can approach the surface of the growing crystal during the growth process [115]. During the growth process the release of latent heat at the surface causes a slight increase in local temperature compared to the solution temperature. This increase in temperature changes the molecular concentration at the surface of the crystal which in turn affects the crystal growth $[115,116]$. Since the formation of zinc ferrite is more exothermic than magnetite [114], more heat will be released during the crystallization, decreasing molecular concentration at the crystal surface and blocking the crystal growth [111, $117]$.

EDX analysis of the samples (Table 2.1) confirmed that actual elemental compositions are relatively in good agreement with nominal zinc values (' $x$ ') in the final products except for the sample with $\mathrm{x}=0.4$. Although composition of this sample deviates from its nominal, the trend in magnetic and structural properties maintained as it will be discussed in the following sections. 


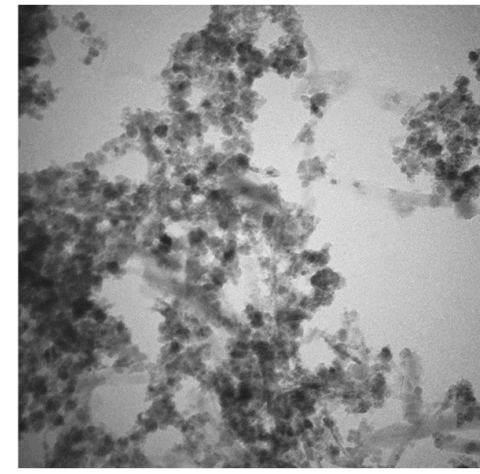

(a)

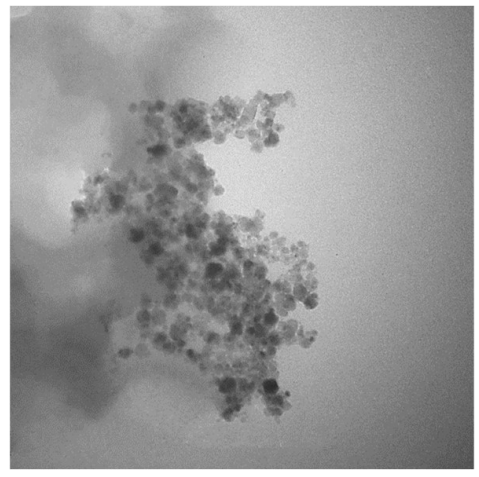

(b)

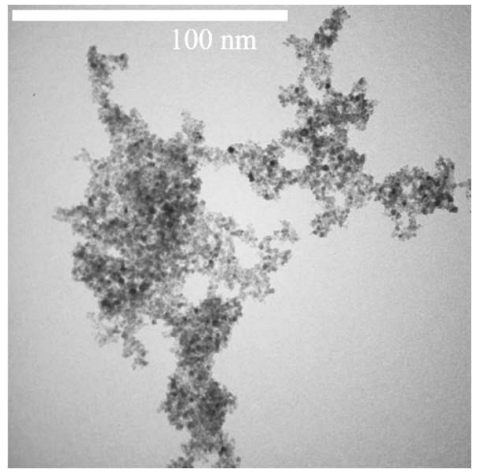

(c)

Figure 2.1TEM images of as-prepared samples a) $\mathrm{x}=0.0, \mathrm{~b}$ ) $\mathrm{x}=0.1$ and $\mathrm{c}$ ) $\mathrm{x}=0.4$ (the scale bar is the same for all images).
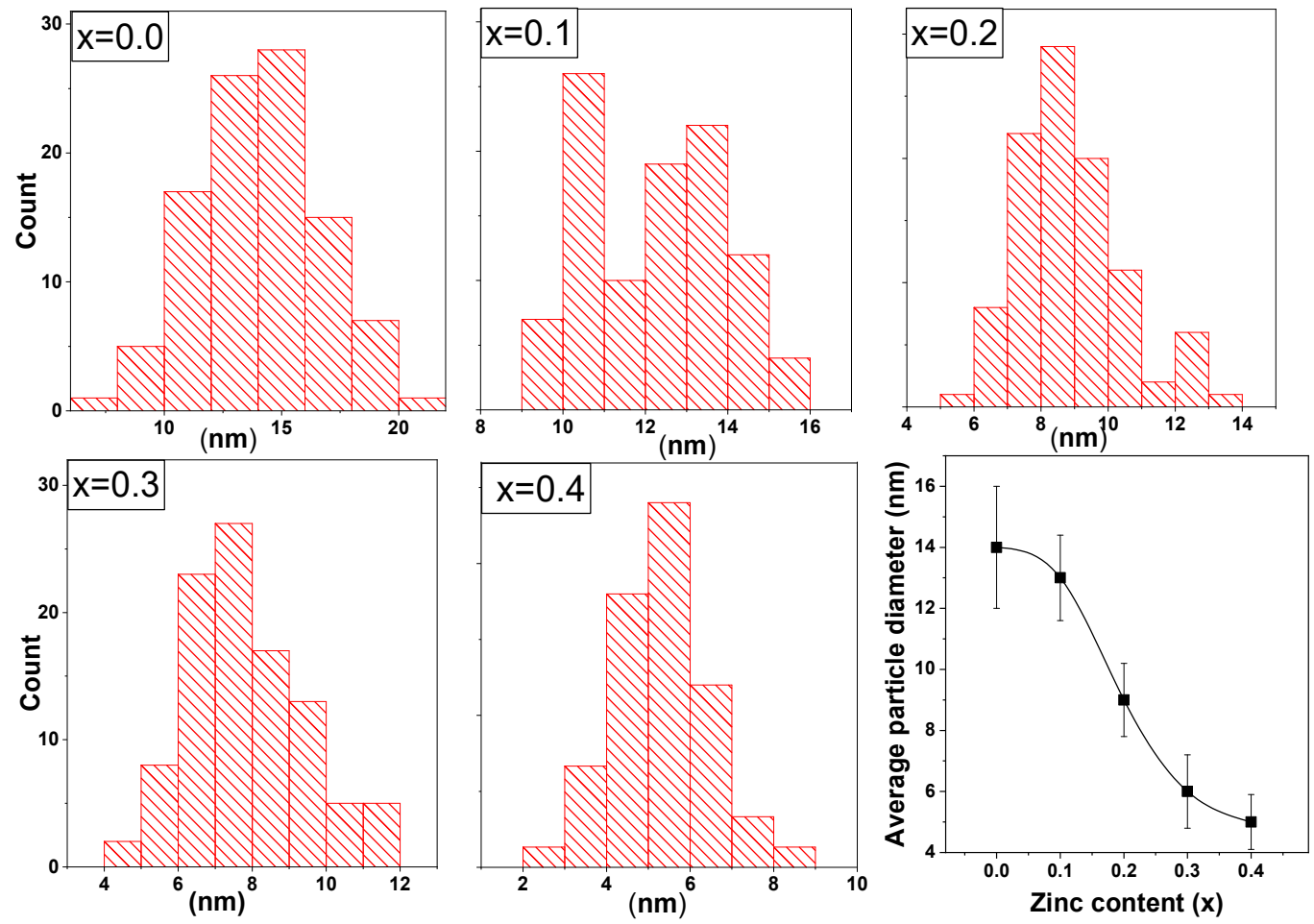

Figure 2.2 Variation of the average particle size with zinc content obtained from TEM images. 
Table 2.1 EXD analysis of nominal compositions $\mathrm{Zn}_{\mathrm{x}} \mathrm{Fe}_{1-\mathrm{x}} \mathrm{Fe}_{2} \mathrm{O}_{4}, \mathrm{x}=0.0,0.1,0.2,0.3,0.4$

\begin{tabular}{c||cc||cc||c}
\multicolumn{1}{c||}{} & \multicolumn{2}{c||}{ Fe (wt. \%) } & \multicolumn{2}{c||}{ Zn (wt. \%) } & $\begin{array}{c}\text { Relative } \\
\text { deviation for Zn } \\
\text { Sample }\end{array}$ \\
\cline { 2 - 5 } & Nominal & EDX analysis & Nominal & EDX analysis & 0 \\
\hline $\mathrm{x}=0$ & 100 & 100 & 0 & 0 & 3 \\
$\mathrm{x}=0.1$ & 96.6 & 96.6 & 3.3 & 3.4 & -1 \\
$\mathrm{x}=0.2$ & 93.3 & 93.5 & 6.6 & 6.5 & -5 \\
$\mathrm{x}=0.3$ & 90.0 & 91.5 & 10.0 & 9.5 & -24 \\
$\mathrm{x}=0.4$ & 86.6 & 89.9 & 13.3 & 10.1 &
\end{tabular}

\subsubsection{Phase identification and XRD analysis (FTIR \& XRD)}

Figure 2.3 shows X-ray diffraction patterns of the as-synthesized Zn-substituted magnetite samples. These diffraction patterns indicate the presence of main peaks related to spinel ferrite structure, (111), (311), (400), (422), (511), (440), and can be assigned to magnetite structure (JCPDS 19-0629). There is an evident peak broadening on the patterns which is related to the small crystallite size of the prepared samples. The average crystallite sizes of the samples (D) were calculated using Scherrer formula $(D=0.9 \lambda / \beta \cos \theta)$, where $\lambda$ is the wavelength of the $\mathrm{X}$-Ray, $\beta$ full width at half-maximum of the peak intensity and $\theta$ is the peak position (Bragg angle) [118]. Considering the most intense peak (311) on the XRD patterns, the estimated crystallite sizes are shown in figure 2.4, which is in good agreement with TEM results. This result shows that each particle is consisted of a crystallite. 


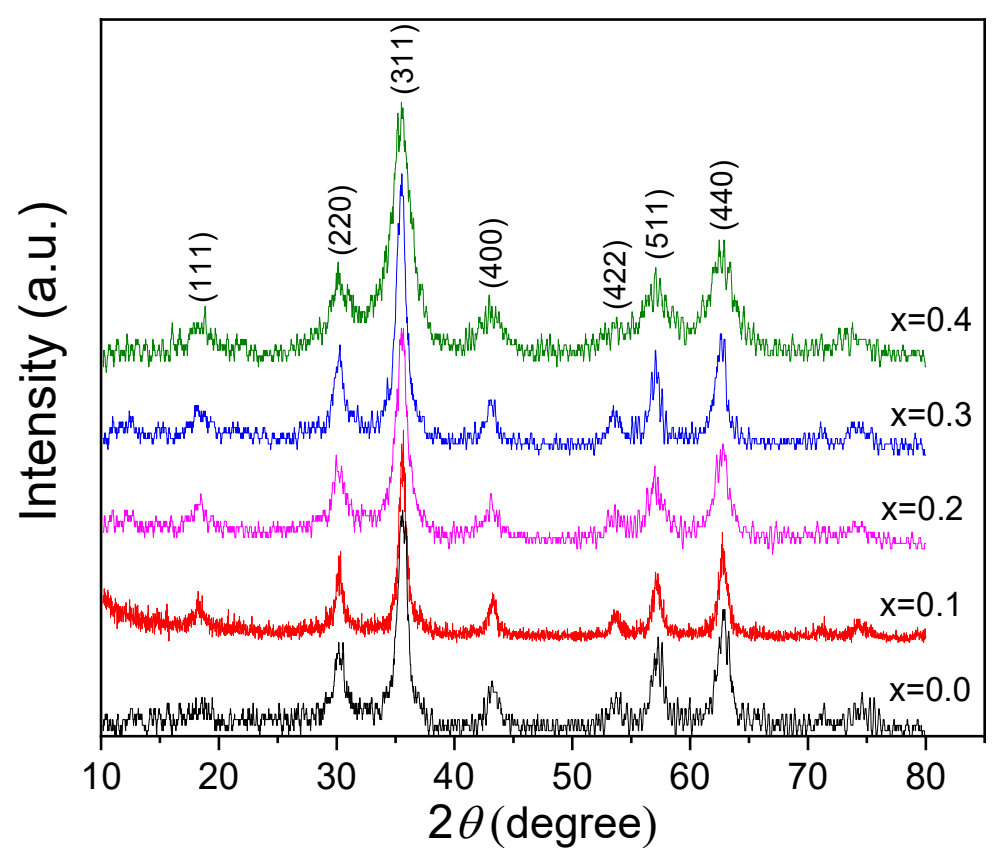

Figure 2.3 X-ray diffraction patterns of synthesized Zn-substituted magnetite nanoparticles. Diffraction peaks are labeled based on the JCPDS 19-0629 standard card.

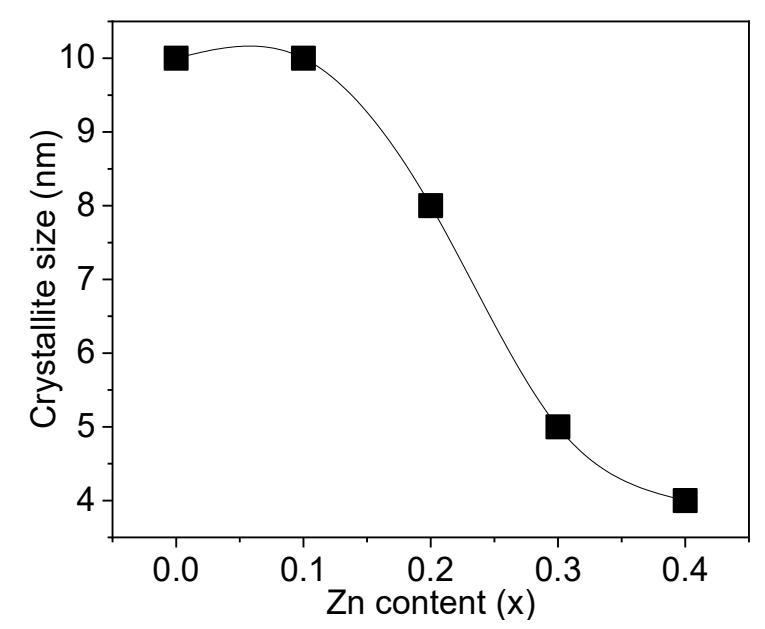

Figure 2.4 Calculated crystallite sizes for different zinc content in magnetite structure.

For all samples the lattice parameter " $a$ " was calculated using Cohen's method [118]. The effect of zinc substitution on lattice parameter is shown in figure 2.5. It is evident that by increasing the zinc content in magnetite structure the lattice parameter has increased. The same behavior has also been reported in different studies on zinc substituted spinel ferrite systems $[111,117,119-121]$. It is well known [122] that one of the parameters affecting " $a$ " in crystal 
structure is the ionic radii of its constituents and the increase in lattice parameter can easily be explained by considering the ionic radii. The ionic radii of $\mathrm{Zn}^{2+}(60 \AA)$ and $\mathrm{Fe}^{2+}$ (63 $\AA$ ) [123] are almost the same, while the ionic radius for $\mathrm{Fe}^{3+}$ cations, which are equally distributed on octahedral and tetrahedral sites, is $49 \AA$ in tetrahedral site [120]. Since zinc has a strong preference to occupy the tetrahedral site, substituting $\mathrm{Fe}^{3+}$ cations in tetrahedral site for $\mathrm{Zn}^{2+}$ cations with larger ionic radius will lead to an expansion of lattice parameter. Generally, in a solid solution of ferrites within the miscibility range, there is a linear relation between the lattice parameter and the concentration of the substituent $[118,119]$. However, the observed nonlinear increased in " $a$ " with zinc content could be due to the fact that octahedral site can also be partially occupied by zinc [124]. On the XRD patterns a shift to lower diffraction angles (the inset in figure 2.5) was observed especially for (440), (511) and (311) diffraction peaks which confirms the lattice parameter expansion by increasing the zinc content [121, 125].

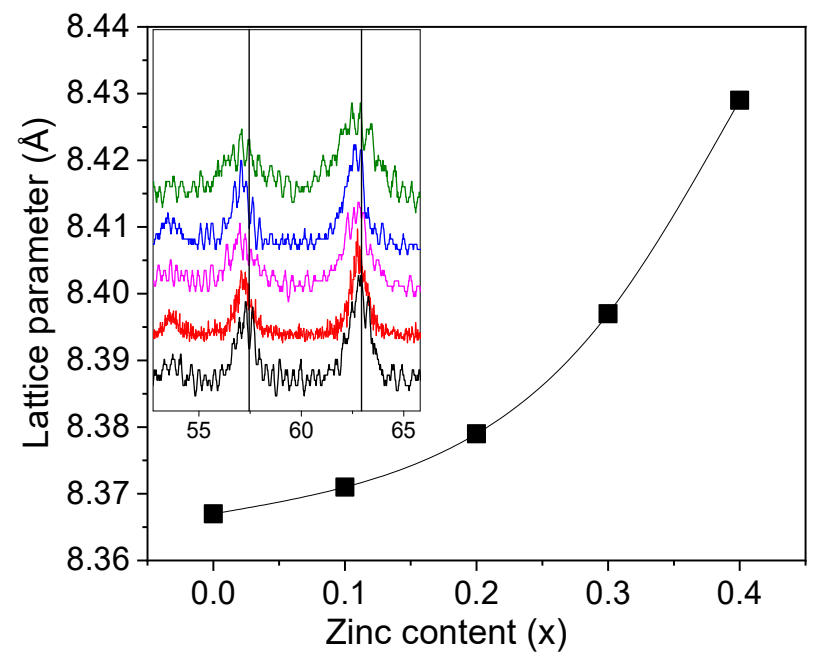

Figure 2.5 The effect of zinc substitution on lattice parameter of magnetite, the inset shows the shit for peaks of (511) and (440) planes to the lower diffraction angles.

It is well-known that magnetite $\left(\mathrm{Fe}_{3} \mathrm{O}_{4}\right)$ and maghemite $\left(\gamma-\mathrm{Fe}_{2} \mathrm{O}_{3}\right)$ have a very similar crystal structure. For this reason, distinguishing magnetite from maghemite only based on the XRD patterns is not easy. On their XRD patterns there are only few differences such as characteristic peaks of maghemite in low angles for $\left(\begin{array}{lll}2 & 1 & 0\end{array}\right)$ and $\left(\begin{array}{lll}2 & 1 & 1\end{array}\right)$ planes which have very weak intensities and are difficult to detect. Nevertheless, the existence of these peaks, in most cases, cannot confirm the existence of a single phase maghemite uniquely [126]. Therefore, FTIR 
measurements were obtained to reconfirm the presence of single phase magnetite. FTIR spectra of magnetite and maghemite have a notable difference around $500-700 \mathrm{~cm}^{-1}$ bands. For these wavenumbers there is only one absorption band for magnetite related to stretching and torsional vibration modes of the Fe-O bonds in tetrahedral and octahedral sites, respectively [127], while for maghemite there are more than one absorption band $[125,128]$. Figure 2.6 shows there is only one absorption band in that region, confirming the sample mainly consisted of magnetite phase. However, surface oxidation of the magnetite nanoparticles and presence of maghemite is almost unavoidable [129]. To minimize the effect of surface oxidation, XRD and FTIR measurements were performed one day after synthesizing the samples. Other absorption peaks around 3400 and $1630 \mathrm{~cm}^{-1}$ are related to $\mathrm{O}-\mathrm{H}$ (hydroxide group) bond and $\mathrm{H}-\mathrm{O}-\mathrm{H}$ stretching vibration modes of water molecules [125].

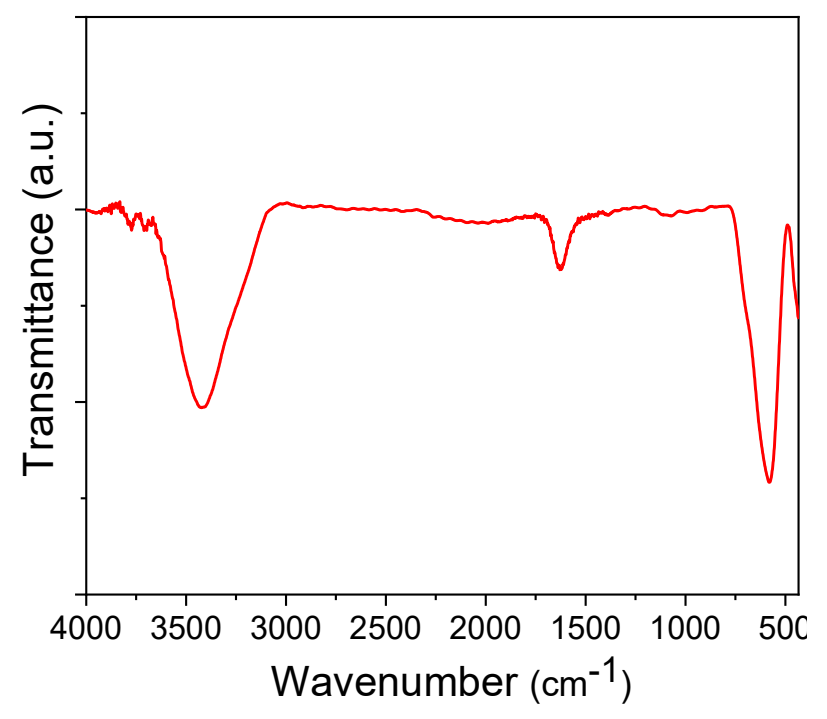

Figure 2.6 FTIR spectra of prepared magnetite nanoparticles.

\subsubsection{Magnetic studies}

Figure 2.7(a) shows room temperature M-H curves of the samples. All samples had a superparamagnetic behavior with zero coercivity due to their small sizes - particles size were below reported critical size for single domain magnetite nanoparticles $49 \mathrm{~nm}$ [24]. Figure 2.7 (b) shows substitution of zinc in magnetite structure drastically enhanced the saturation magnetization of for $\mathrm{x}=0.1$. 


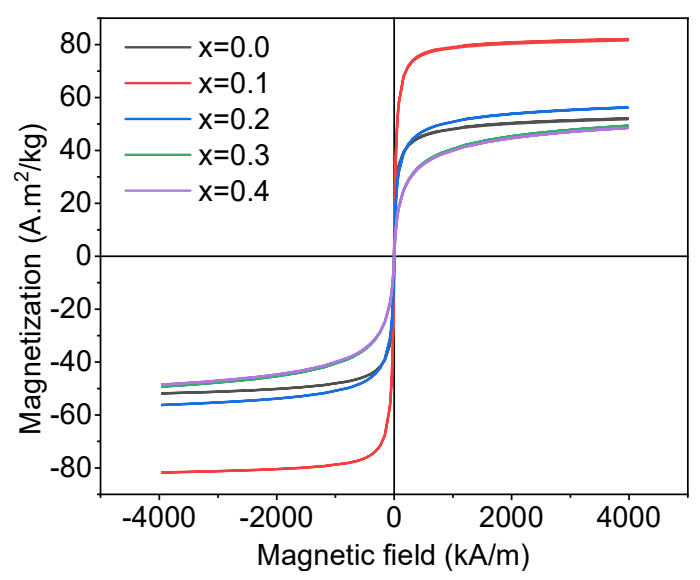

(a)

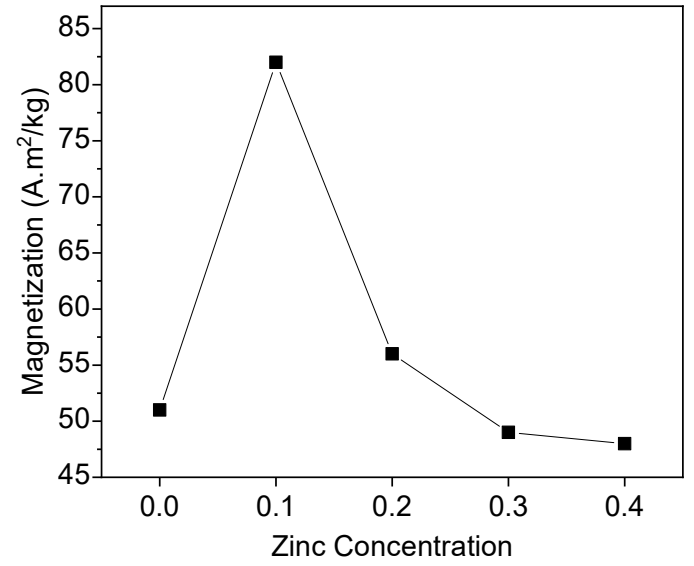

(b)

Figure 2.7 (a) Room temperature M-H curves of the samples, (b) the effect of zinc substitution on saturation magnetization of as-prepared samples.

Stoichiometric bulk magnetite has inverse spinel structure at room temperature, which means all $\mathrm{Fe}^{2+}$ cations are located on octahedral (B) sites while $\mathrm{Fe}^{3+}$ cations are equally distributed on tetrahedral and octahedral sites. Theoretically, one can calculate the saturation magnetization of magnetite knowing the magnetic moment of each cation, their distribution on the different sites of crystal and considering the fact that the interaction between tetrahedral and octahedral sites is negative. Although all $\mathrm{AB}, \mathrm{AA}$ and $\mathrm{BB}$ interactions tend to be negative, they cannot be all simultaneously negative [18]. As pointed out by Neel [130], in bulk magnetite structure the antiparallel alignment of the moments on the $\mathrm{A}$ and $\mathrm{B}$ sites would result in cancelation of $\mathrm{Fe}^{3+}$ magnetic moments; therefore, the net magnetization arise from $\mathrm{Fe}^{2+}$ cations on the octahedral sites $\left(4 \mu_{\mathrm{B}}\right.$ per unit formula) [18]. Saturation magnetizations of the nanoparticles are usually lower than their bulk counterparts when the cation distribution configuration is the same [131]. For magnetite nanoparticles prepared with different methods a wide range of saturation magnetizations have been reported. Although typical values range from 30 to $50 \mathrm{~A} . \mathrm{m}^{2} / \mathrm{kg}[132$, 133], some studies have reported higher values such as $82 \mathrm{~A} \cdot \mathrm{m}^{2} / \mathrm{kg}$ [134] and $114 \mathrm{~A} \cdot \mathrm{m}^{2} / \mathrm{kg}$ [37]. The reduction in saturation magnetization can occur due to different phenomena such as existence of noncollinear spins at the surface $[132,135,136]$, the presence of an amorphous layer around the particles or surface oxidation [137] defects at the particle surface [132], finite size effects [138] and non-saturation effects because of random distribution of the small particles with enhanced values of magneto-crystalline anisotropy [131, 139]. 
It has been reported in different studies $[37,120,140,141]$ that $\mathrm{Zn}^{2+}$ has a strong affinity to occupy the tetrahedral site in spinel structure. Since $\mathrm{Zn}^{2+}$ is a diamagnetic cation with no magnetic moment $0 \mu_{\mathrm{B}}$, this weakens the antiferromagnetic coupling interactions between $\mathrm{Fe}^{3+}$ cations on A and B sites, hence would result in larger net magnetization in the system. However, at very high contents of $\mathrm{Zn}^{2+}$ in magnetite structure, A site moments will become too weak to affect $\mathrm{B}$ moments. Therefore, antiferromagnetic coupling interactions between $\mathrm{Fe}^{3+}$ ions become dominant, decreasing the net moment $[18,37,112,120]$. However, this increase and then decrease trend in saturation magnetization by substituting zinc ion in ferrites, which has been reported for different percentages of zinc substitution [37, 112, 120, 121, 142-145], is mainly due to different cation distributions which in turn changes the strength of the superexchange interactions among magnetic ions $[66,146]$. Indeed, when zinc substitution is high, B moments are not completely antiparallel to the A ones and there will be a spin canting in the ferrite structure which leads to a reduction in net magnetization. The spin canting is maximum when zinc cations are substituted in A sites and is less pronounced when zinc cations are simultaneously substituted in A and B sites $[66,120]$.

Figure 2.8 shows the zero-field-cooling (ZFC) measurements, plotting the imaginary component of the AC susceptibility versus temperature using the same VSM unit. As it was expected, all samples had a blocking temperature $\left(\mathrm{T}_{\mathrm{B}}\right)$ below room temperature which confirms the superparamagnetic behavior of the samples at room temperature. $T_{B}$ is the temperature where the thermal activation energy overcomes the magnetic anisotropy energy barrier of a single domain magnetic nanoparticle, causing the fluctuation of the moments between two directions of its easy axis. Below $\mathrm{T}_{\mathrm{B}}$, the hysteresis loop presents remanence and coercivity; these two parameters are enhanced for lower temperatures and vanish above $T_{B}[24]$. 


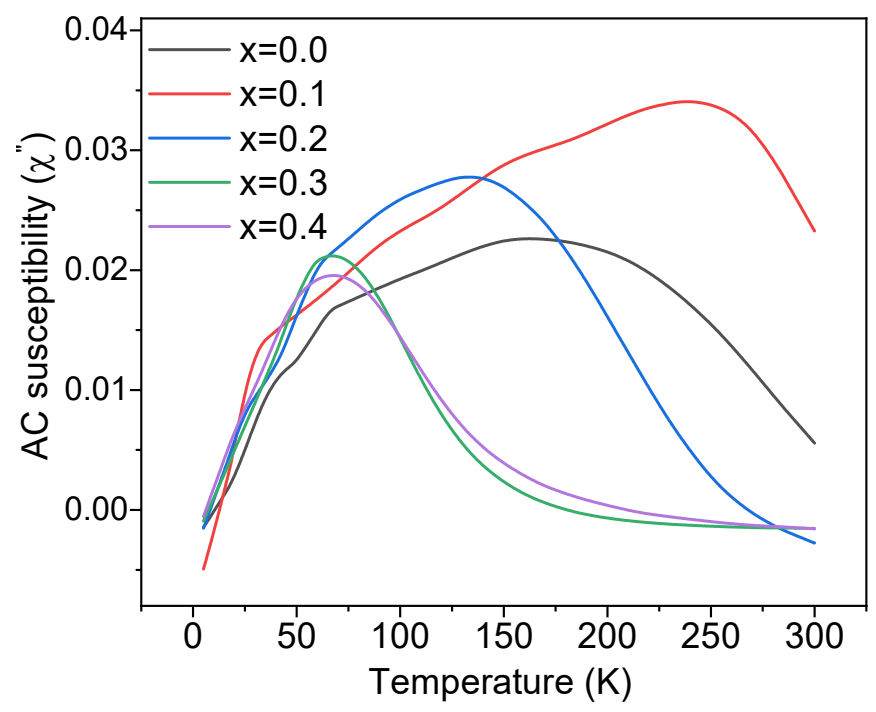

Figure 2.8 Zero-field-cooling (ZFC) measurements of the samples by plotting the imaginary component of the AC susceptibility with respect to temperature

According to Stoner-Wohlfarth single domain theory [147], the magnetic anisotropy energy, holding the magnetic moments in an easy direction, can be approximated by:

$$
E=K V \sin ^{2} \phi
$$

where $K$ is the effective magnetocrystalline anisotropy constant, $V$ is the nanoparticle volume and $\phi$ is the angle between magnetic moment and easy axis of the nanoparticle directions. At a zero applied magnetic field, two easy directions are separated by the energy barrier $(E=K V)$. When magnetic anisotropy energy is much greater than the thermal energy $\left(k_{B} T\right)\left(K V \gg k_{B} T\right)$, the magnetic moment cannot flip spontaneously. Therefore, in this case the system behaves as a ferromagnet. On the other hand, if the thermal energy is of the order of anisotropy energy $\left(K V \sim k_{B} T\right)$ or less, spontaneous switching of the magnetic moments between two directions of easy axis can occur on the timescale of a measurement. This spontaneous relaxation, caused by the thermal energy, is referred as superparamagnetic state [26]. As stated before, the magnitude of $(E)$ determines the blocking temperature in which thermal activation overcomes the anisotropy energy barrier and the system experiences a transition to superparamagnetic state. Equation (2.1) shows that anisotropy constant $(K)$ and nanoparticle volume $(V)$ determine the magnitude of this barrier, however, the observed blocking temperature of the system strongly depends on the measurement timescale as well. When this timescale is larger than the relaxation time of the magnetic moments, at a given temperature, superparamagnetism behaviour can be observed, otherwise a hysteresis loop will be observed in the system with exactly the same value of the energy barrier [24]. Therefore, it is important to note that the blocking temperature 
is not an absolute temperature, it depends not only on the intrinsic magnetic properties of the nanoparticles but also on the timescale of the measurements. As it can be seen, there is a clear peak broadening in ZFC measurements of our samples which by considering the role of all aforementioned parameters in determining the blocking temperature, may indicate that the polydispersity in particle sizes can cause this broadening — from TEM images this relative polydispersity is evident.

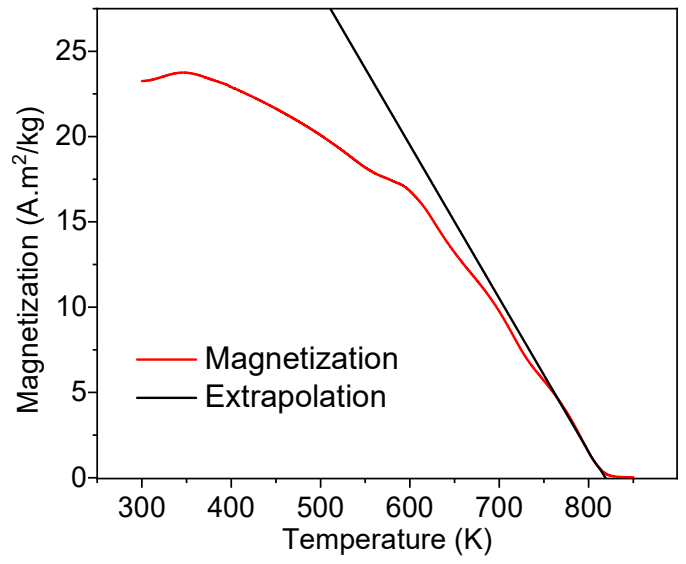

(a)

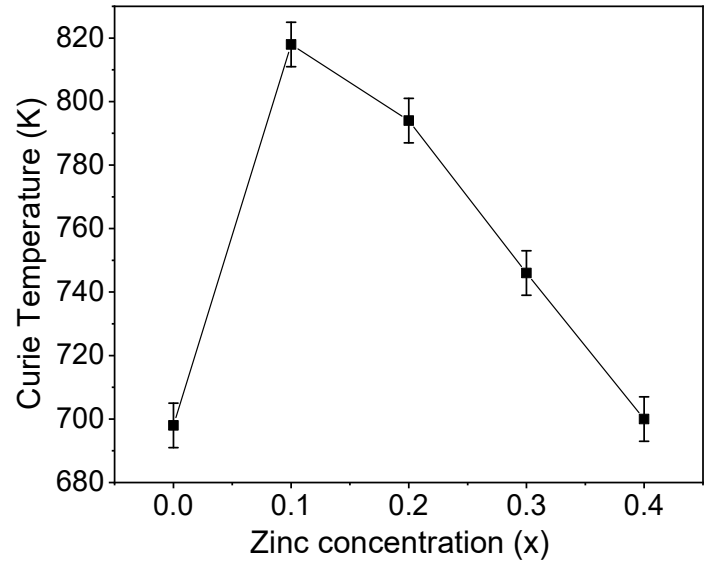

(b)

Figure 2.9 A typical extrapolation of curves for the variation of magnetization with respect to temperature (a) and observed Curie temperature of the samples (b).

For better understanding the magnetic properties of the prepared nanoparticles, we studied their behavior at higher temperatures by plotting the variation of magnetization with respect to temperature and determined their Curie temperature. Curie temperatures were obtained using two different methods: (i) extrapolation of the main parts of the curves [18], where the magnetization tends to zero and (ii) two tangent method [148]. Figure 2.9(a) shows a typical extrapolation of the curve for the sample with $x=0.1$ and figure 2.9 (b) shows the mean Curie temperature obtained by the two methods as a function of zinc content. The difference between these two values, was not higher than $7 \mathrm{~K}$, therefore, $\pm 7 \mathrm{~K}$ was adopted as error bars in the graph. Curie temperature highly depends on the number and strength of magnetic interactions between cations through oxygen anions per unit formula and also on their distribution over A and B sites $[125,149]$. That number decreases when diamagnetic cations are substituted for paramagnetic ones in the spinel structure, leading to a reduction in Curie temperature [66]. Indeed, substitution of diamagnetic cations, here $\mathrm{Zn}^{2+}$, reduces the internal energy for removing 
the spin alignment, resulting in a reduction in the Curie temperature. This can be explained by the fact that A-B superexchange interaction, resulting from replacements of paramagnetic $\mathrm{Fe}^{3+}$ by diamagnetic $\mathrm{Zn}^{2+}$ ions at tetrahedral site, decreases which in turn reduces both saturation magnetization and Curie temperature.

Such a decrease in Curie temperature with increasing zinc content was expected and is in agreement with previous studies [150-152]. However, this trend was not observed in transition from $x=0.0$ (magnetite) to the sample $x=0.1$. It is well-known that the Curie temperature of bulk magnetite is about $850 \mathrm{~K}[132,137]$, but in nanoscale considerable smaller values such as $704 \mathrm{~K}$ [102], $713 \mathrm{~K}$ [153] and $738 \mathrm{~K}$ [154] have also been reported. Manohar et al. [153] attributed the smaller observed Curie temperature to several phenomena such as the presence of higher amount of canted magnetic spins on the surface and size effects. On the other hand it is well-known that magnetite has an unstable structure at temperatures above $250{ }^{\circ} \mathrm{C}[102,155$, 156]. Therefore when studying the Curie temperature of magnetite a great caution should be exercised. Figure 2.10 shows the variation of the magnetization with respect to temperature for sample $\mathrm{x}=0.0$. As it can be clearly seen, after that magnetization drops almost to zero, there is a cusp on the curve. This trend was seen in some other ferrites too [157-159] and can be related to irreversible changes in the crystal structure. These changes includes either cation redistribution from a metastable to a stable state [157] or a phase transition as in magnetite to maghemite and to hematite [102]. Hence, according to figure 2.10 we believe, in our case, the first drop to zero magnetization can be considered as corresponding Curie temperature or may be more properly referred as phase transition temperature [154] rather than Curie temperature. However, if one consider the second drop in the cusp as Curie temperature $(830 \mathrm{~K})$, its value still is higher than that of the sample $\mathrm{x}=0.1(818 \mathrm{~K})$ and yet lower than that of bulk magnetite. 


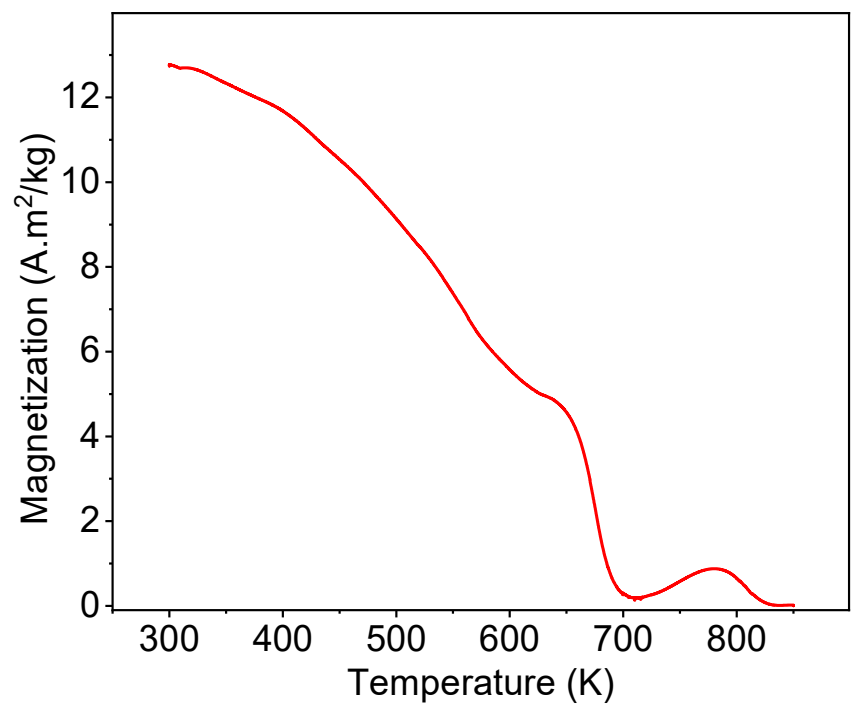

Figure 2.10 The variation of magnetization with respect to temperature for sample with $\mathrm{x}=0$ (magnetite).

\subsection{Summary}

Zinc substituted magnetite $\left(\mathrm{Zn}_{\mathrm{x}} \mathrm{Fe}_{1-\mathrm{x}} \mathrm{Fe}_{2} \mathrm{O}_{4} \mathrm{x}=0,0.1,0.2,0.3\right.$ and 0.4$)$ nanoparticles were synthesized by simple coprecipitation method and all samples were in superparamagnetic state, a key factor in MMUS application. Results showed that incorporation of zinc in magnetite structure can remarkably enhance its saturation magnetization while maintaining the cubic spinel structure of the host (i.e. magnetite). Since, it has been already demonstrated [37] zinc substituted magnetite nanoparticles are nontoxic to healthy cells, this enhancement in magnetic properties of magnetite nanoparticles by zinc can open the possibility of its application in in vivo studies not only as diagnostic agent (contrast agent in medical imaging), but also as a therapeutic agent in magnetic hyperthermia simultaneously (theranostic agent). 


\section{Chapter 3 : Magnetomotive ultrasound imaging using zinc substituted magnetite nanoparticle}

\subsection{Introduction}

In recent decades, medical imaging techniques have been extensively developed to enhance diagnosis methods, detect the pathologies, and monitor the effect of the therapy procedures. The development of minimally invasive, real-time, non-ionizing, portable, and cost-effective medical imaging techniques is of major concern. Most of the conventional imaging modalities only provide macroscopic physical and morphological differences between the normal and pathological tissues. However, visualizing the events at molecular and cellular level can significantly enhance the diagnosis and consequently the effectiveness of the treatment. As early and accurate detection is a key factor for survival rate for diseases such as cancer.

Optical-based imaging modalities have been used to acquire molecular images with high resolution and acceptable sensitivity; however, the penetration depth in these type of imaging modalities is a limitation [160-162]. Radionuclide imaging techniques (PET and SPECT) using radioactive elements that can be linked to a specific relevant molecule are also capable of visualizing physiological activity, though limited spatial and temporal resolution and the use of radioactive materials are two important concerns in these techniques [162, 163]. Magnetic resonance imaging (MRI) among other modalities has several advantages. It is non-ionizing, high resolution, and has multiple contrast mechanisms, providing anatomical, physiological, cellular, and molecular information. Moreover, several types of magnetic nanoparticles have been developed as probes for imaging specific molecular pathways related to different type of diseases. [164]. However, MRI is an expensive and non-portable method with long examination time, temporal resolution in the order of seconds, and cannot provide real-time molecular and cellular information $[165,166]$.

Ultrasound is one of the most widely used diagnostic imaging techniques, as one fourth of all medical imaging worldwide is conducted using ultrasound [167]. It uses non-ionizing radiation, provides real-time images, and as long as high intensities are not used, to the best of present knowledge can be considered as a safe method. Compared to other techniques with similar functionality, it is more cost-effective and, therefore, widely available and also portable. 
Its resolution is in the millimeter range and may be improved if the frequency is increased. It can provide blood flow information through Doppler mode. Finally, since acoustic wave propagation depends strongly on the medium, a wide variety of parameters such as speed of sound, acoustic impedance, dispersion, attenuation, elasticity, nonlinearity, etc. can be measured. These parameters can be employed to map and characterize the tissue or to measure the internal temperature. However, it has some important limitations such as being operator dependent, difficulties in obtaining information from organs containing gases and bony structures, and for organs such as heart and brain only limited windows are available. In addition, its efficiency to detect biomarkers for molecular imaging is limited. Ultrasound resolution is low for the directly visualization of nanoparticles and the backscattering crosssection of a single magnetic nanoparticle or even an agglomeration of nanoparticles is weak to allow adequate contrast between nanoparticles and surrounding tissue [167-169].

Recently [44], magnetomotive ultrasound (MMUS) has been introduced as an imaging technique that uses ultrasound echo signals to localize magnetic nanoparticles within tissues by detecting microvibrations originated from the interaction of the particles with an external oscillating magnetic field. These nanoparticles microvibrations and consequently in their surrounding tissues is detected by a high frame rate ultrasound imaging and thus mapping the distribution of the nanoparticles. MMUS has been, for example, used to detect sentinel lymph nodes in rats [170]. In addition, assessing molecular events in the body can be potentially achieved by using targeted magnetic nanoparticles. This technique is also an alternative to estimate viscoelastic properties of soft tissues [47, 171].

It is worth mentioning that in the case of detecting magnetic nanoparticles within tissues, a variety of imaging modalities such as magnetic resonance imaging [37] and magnetic particle imaging [172] have been proven to localize magnetic nanoparticles; however, these techniques are neither cost effective nor portable and are still limited in generating real-time images. Alternate current biosusceptometry is another cost-effective and portable method to detect magnetic nanoparticles within tissue [173]; however, it has limitations in spatial resolution. To improve this drawback, a hybrid transducer was developed, which is capable of acquiring susceptometric and MMUS data, simultaneously [174]. An In vivo study showed the feasibility of using this hybrid transducer to monitor gastric emptying [175].

In MMUS imaging, one of the main conditions to improve the signal noise ratio (SNR) is to use superparamagnetic nanoparticles with enhanced saturation magnetization [48]. In [48], the 
authors used zinc-doped magnetite nanoparticles with enhanced magnetization to increase the magnetomotive force, consequently, improving the MMUS detected signal. Their particles were synthesized by a thermal decomposition method as described in [37]. In the present study, Zn-substituted magnetite nanoparticles $\left(\mathrm{Zn}_{\mathrm{x}} \mathrm{Fe}_{1-\mathrm{x}} \mathrm{Fe}_{2} \mathrm{O}_{4}, \mathrm{x}=0,0.1,0.2,0.3\right.$ and 0.4$)$ prepared by a cost-effective and simple coprecipitation method were evaluated as contrast agent in MMUS imaging.

\subsection{Materials and Methods}

\subsubsection{MMUS experiments}

The magnetic energy of a particle with a permanent magnetic moment $\mu$ in an external magnetic field $\mathrm{B}(\mathrm{r})$ can be described as [176]:

$$
U(r)=-\vec{\mu} \cdot \overrightarrow{\mathrm{B}}(\mathrm{r})
$$

The force acting on the particle is, then, equal to the negative gradient of this potential:

$$
\vec{F}=-\nabla U
$$

However, for superparamagnetic nanoparticles the magnetic moment is the due to the applied magnetic field and it is described as:

$$
\vec{\mu}=\mu(H)=\vec{M}(H) V=\chi V \vec{H}(r) .
$$

The magnetic energy, therefore, is given by

$$
U=\vec{\mu}(H) \cdot \vec{B}
$$

In previous studies $[44,177]$, it has been demonstrated that in an external magnetic field with a flux density of $B=\sin \left(\omega_{0} t\right) B_{Z}(z)$ in $\mathrm{z}$ direction and angular frequency $\omega_{0}$, the one dimensional magnetic force $F(z)$ acting on a superparamagnetic nanoparticle of volume $V$ can be expressed as:

$$
F(z)=\left(\frac{\chi V}{2 \mu_{0}}\right)\left(1-\cos \left(2 \omega_{0} t\right)\right) B_{z}(z) \frac{d B_{z}(z)}{d z}
$$

where $\chi$ is the magnetic susceptibility of the nanoparticle and $\mu_{0}$ is the magnetic permeability of the free space. This equation shows that the force is linearly proportional to the magnetic susceptibility of the nanoparticles as well as the magnitude and the gradient of magnetic flux 
density. It should also be noted that the oscillation frequency of the nanoparticles, due to this force, is two times larger than that of the magnetic field frequency. This relation can be considered as a reliability of the observed magnetomotive response of magnetic nanoparticles in the medium due to the magnetic field [177]. Equation (3.5) describes the magnetomotive force acting on magnetic nanoparticles, however, the observed displacement in MMUS strongly depends on viscoelastic properties of the medium surrounding the nanoparticles. Since the nanoparticles are immersed in a viscoelastic medium (tissues or phantom), the net force will be the sum of magnetomotive force with an elastic restoring force $(k z(t))$ and a viscous drag force $(r \partial z / \partial t)$ arising from the medium as $[44,178]$ :

$$
F(z)=\left(\frac{\chi V}{2 \mu_{0}}\right)\left(1-\cos \left(2 \omega_{0} t\right)\right) B_{z}(z) \frac{d B_{z}(z)}{d z}-k z(t)-r \frac{\partial z}{\partial t} .
$$

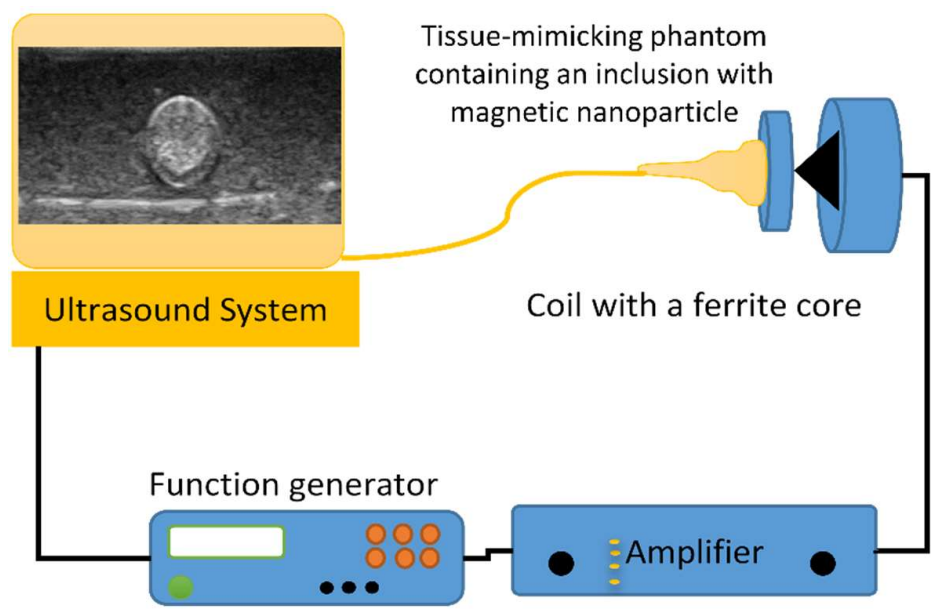

Figure 3.1 Schematic depiction of the experimental setup for MMUS experiments. A low frequency magnetic field is applied to the phantom containing an inclusion with magnetic nanoparticles and the induced microvibrations is detected using ultrasound imaging.

The schematic depiction of the setup for MMUS experiments is illustrated in figure 3.1. The system was consisted of an excitation coil (Solen Inc., Saint-Hubert, QC, Canada) with $9.1 \mathrm{mH}$ of inductance, $2.7 \Omega$ impedance, $22 \mathrm{~mm}$ height and inner and outer diameters of $45 \mathrm{~mm}$ and 89 $\mathrm{mm}$, respectively. To increase the magnitude and the gradient of the applied magnetic field at the inclusion region a ferrite flux concentrator core was positioned at the center of the coil, 2 $\mathrm{mm}$ away from the phantom's surface. The flux concentrator had $50 \mathrm{~mm}$ in height, $15 \mathrm{~mm}$ in diameter at the base, and $3 \mathrm{~mm}$ in diameter at the tip. The coil was driven by a function 
generator (Agilent, model 33522A) and a power amplifier (Ciclotron, model Dynamic 8000 $\Omega 2$, class $\mathrm{AB}-2,000 \mathrm{~W}$ ). Tone bursts (10 cycles) of sinusoidal voltage with frequency of 50 $\mathrm{Hz}$ were used to produce the magnetic field. The interaction between the magnetic field and the magnetic nanoparticles induces the magnetic force, resulting in vibration within the phantom. The magnetic field amplitude in the region containing the nanoparticles, $5 \mathrm{~mm}$ from the tip of the ferrite core of the coil, was $0.1 \mathrm{~T}$ (measured by a GLOBALMAG, TMAG-1T, gauss meter). A Sonix RP (Ultrasonix, Richmond, BC, Canada) ultrasound scanner, with a linear ultrasound transducer (L14-5/38) operating at $9.5 \mathrm{MHz}$ positioned opposite the coil, was used. The ultrasound data were acquired at a pulse repetition frequency of $2 \mathrm{kHz}$ and each frame was composed of 128 scan-lines. To acquire frames at this high temporal resolution, a sector subdivision technique was used [179].

MMUS images were obtained by tracking the axial displacements induced to the internal parts of the phantom due to the interaction of the external magnetic field with the nanoparticles. Briefly, the displacement was measured by applying a speckle tracking method, using the cross-correlation technique, to consecutive ultrasonic echoes acquired while the magnetic field was on. From the cross-correlation output and using the sampling frequency information, the time-delay between the pair of echoes was estimated and then converted into displacement. For more details about the MMUS platform used in this experiment and the algorithm used to estimate displacement, the reader is referred to [180].

\subsubsection{Phantom preparation}

MMUS experiments in this study were conducted using two different tissue-mimicking phantom materials; oil-based gel and water-based (gelatin).

Oil-based phantoms. To prepare the oil-based gel phantoms, the copolymer styreneethylene/butylene-styrene (SEBS) in mineral oil was used to manufacture translucent gels presenting acoustic and elastic properties similar to tissue [181, 182]. Phantoms of SEBS gel present excellent temporal stability in terms of elastic and acoustic properties [181, 182], allowing good flexibility in performing the experiments.

The phantoms' molds were cylinders with $5 \mathrm{~cm}$ in diameter and $2.5 \mathrm{~cm}$ in height. To prepare the phantoms a mixture of SEBS (Kraton G 1650 M, Kraton Polymers, Paulinia, SP, Brazil) and mineral oil were heated to $130{ }^{\circ} \mathrm{C}$ and stirred in a vacuum oven (YK Oven Vac4, YK Tecnologia, Sapiranga, RS, Brazil) until a completely homogeneous mixture was obtained. The obtained gel was kept for 30 more minutes in the vacuum oven, at $130{ }^{\circ} \mathrm{C}$, to remove any 
existing gas bubbles. To manufacture the inclusion, first glycerol was mixed with the nanoparticles and sonicated for 20 minutes. This glycerol/nanoparticles mixture was added to the gel at $85{ }^{\circ} \mathrm{C}$ in a fraction of $15 \%$ of the oil mass. This procedure $[183,184]$ ensures that these nanoparticles are located in homogeneously dispersed glycerol droplets formed in the phantoms. The reader is referred to the ref $[183,184]$ for details about the preparation of glycerol in oil-based phantoms as well as the acoustic and elastic properties of the materials. At this temperature, five hemisphere $(1 \mathrm{~cm}$ in diameter $)$ inclusions each consisting of $30 \mu \mathrm{mol}$ $(\approx 0.7$ wt.\% of the inclusion mass) of one type of prepared magnetic nanoparticles were manufactured. Each of the inclusions was placed in the center of the phantoms' molds and then they were filled with the remaining gel to shape a cylindrical phantom with a hemisphere inclusion at the center. Glass microspheres $(>38 \mu \mathrm{m}$ in diameter, CMV, Cachoeirinha, RS, Brazil) were added to the background material, in a concentration of $0.5 \%$ in mass, to promote ultrasonic scattering.

Water-based gelatin phantoms. To prepare the water-based phantoms a solution of $6 \mathrm{wt}$. \% gelatin (Gelita, Sao Paulo, Brazil) was heated to $70^{\circ} \mathrm{C}$ and then cooled to $30^{\circ} \mathrm{C}$. Then, formaldehyde was added to the solution with mass equal to $5 \%$ of the mass of the gelatin. Using the same gelatin solution, inclusions containing $34 \mu \mathrm{mol}(0.78 \mathrm{wt} . \%$ of the inclusion) of magnetic nanoparticles were prepared and inserted in the center of each phantom. Inclusions were semi spheres with $1 \mathrm{~cm}$ diameter. The nanoparticles were added to the solution at $35^{\circ} \mathrm{C}$. The phantoms were cylinders with $5 \mathrm{~cm}$ diameter and $2.5 \mathrm{~cm}$ height. Five phantoms with the same geometries were manufactured, where the inclusions of each phantom was manufactured using different types of prepared nanoparticles.

\subsection{Results and Discussion}

A typical B-mode and MMUS images of the oil-based phantoms with magnetic nanoparticle inclusion are shown in figure 3.2. This MMUS image shows displacements of up to $7 \mu \mathrm{m}$. The regions presenting higher displacements indicate where the nanoparticles are located. The displacements caused by the interaction of different samples with the applied magnetic field in a same situation, $90 \mathrm{~ms}$ (time for one of the displacement peaks) after turning the magnetic field on, were compared. Figure 3.3 (a) shows the observed peak displacement in the center of the inclusions for each phantom prepared with different magnetic nanoparticles.

(a) 

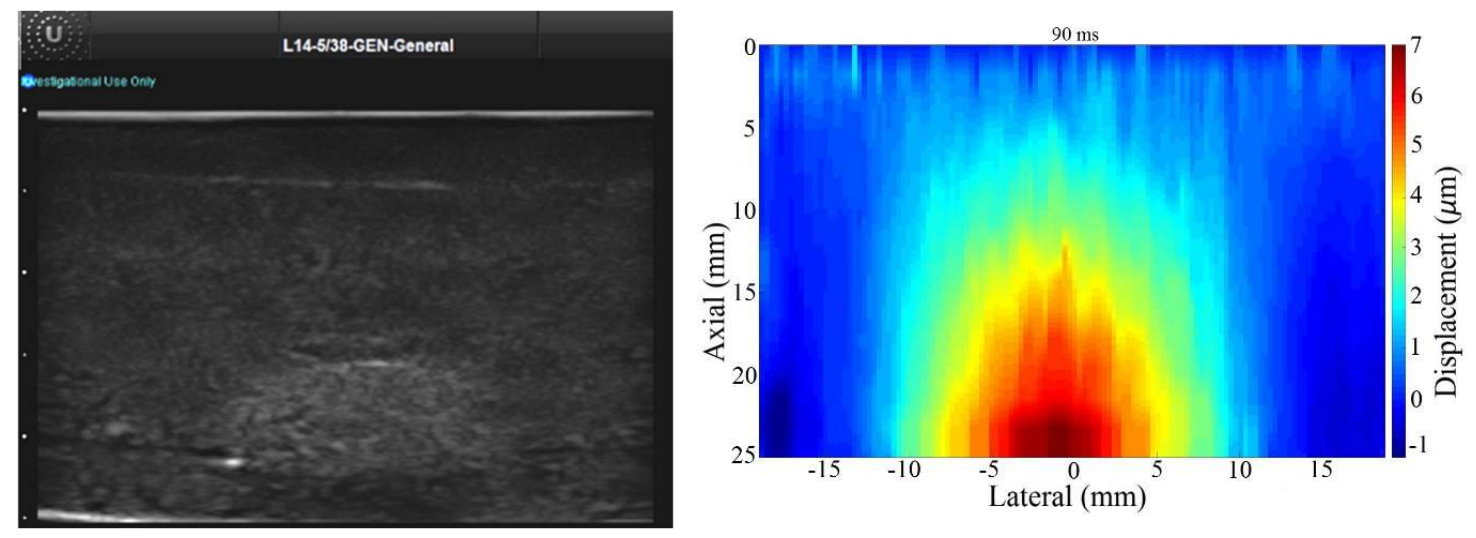

Figure 3.2 A typical B-mode image of the phantoms with magnetic nanoparticle $(x=0.1)$ inclusion (a) and its MMUS image (b).

As can be clearly seen, the displacement increased significantly in the case of nanoparticles with $\mathrm{x}=0.1$ (twofold increase compared to the pure magnetite). Since the maximum magnetic field produced by our system was $0.1 \mathrm{~T}(\sim 79 \mathrm{kA} / \mathrm{m})$, the magnetization values were determined in $\mathrm{M}-\mathrm{H}$ curves at the same magnetic field (Figure 3.3 (b)). Comparing the changes in magnetization at this field, for different zinc content in magnetite structure with observed displacements, one can obviously see the direct role of magnetization or magnetic susceptibility on the induced magnetic force. Although the amount of magnetic nanoparticles used in each phantom were the same, the phantom with the inclusion made out of the sample $x=0.1$ had the highest MMUS signal. There is at least one other study [48] on improving the MMUS signal by optimizing the magnetic properties of the contrast agents using the strategy of doping zinc in magnetite structure. In that study, the authors used the thermal decomposition method to manufacture the particles and reported the highest saturation magnetization of the resultants for $\mathrm{x}=0.4$. For that zinc content they observed a surprising value of $175 \mathrm{~A} \cdot \mathrm{m}^{2} / \mathrm{kg}$, which is higher than what we observed in the present study. However, in terms of cost and simplicity of the synthesis method (i.e. thermal decomposition), coprecipitation is remarkably simpler and cost-effective. It should also be noticed that magnetic nanoparticles produced by coprecipitation tend to show broader particle size distribution and lager aggregates [185]. Those parameters have been shown to play a role in MMUS output as well [186]. 


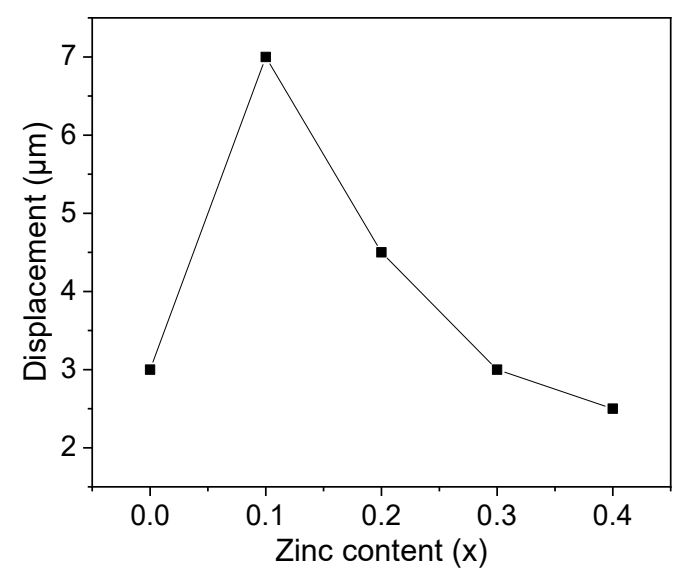

(a)

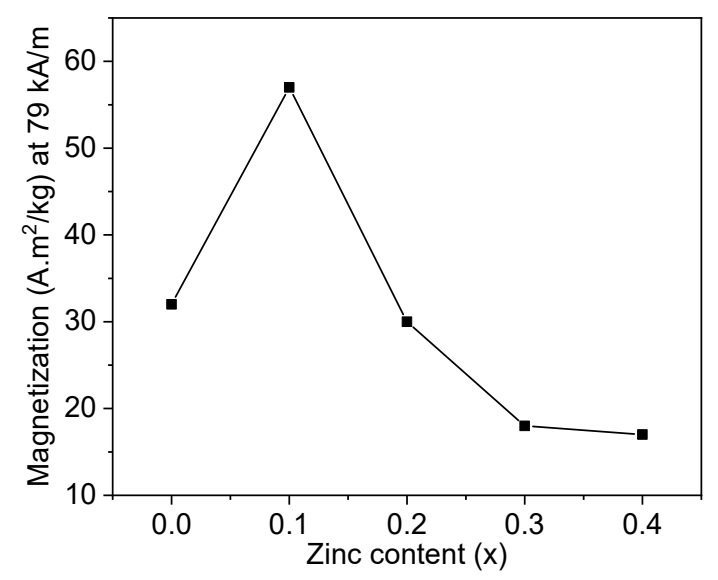

(b)

Figure 3.3 Observed displacement in the inclusions for each phantom prepared with different magnetic nanoparticles (a) and magnetization values at applied magnetic field of $0.1 \mathrm{~T}$ for different zinc concentration in magnetite structure (b).

Figure 3.4 shows the displacement observed for gelatin phantoms. Although, the applied magnetic field strength and profile was the same for both experiments, clearly, for all magnetic nanoparticles the maximum displacement has increased drastically compared to the oil-based phantoms. This difference can be explained because phantoms manufactured at the gelatin concentration $[187,188]$ used here are considerably softer than the SEBS gels manufactured in the present study [183].

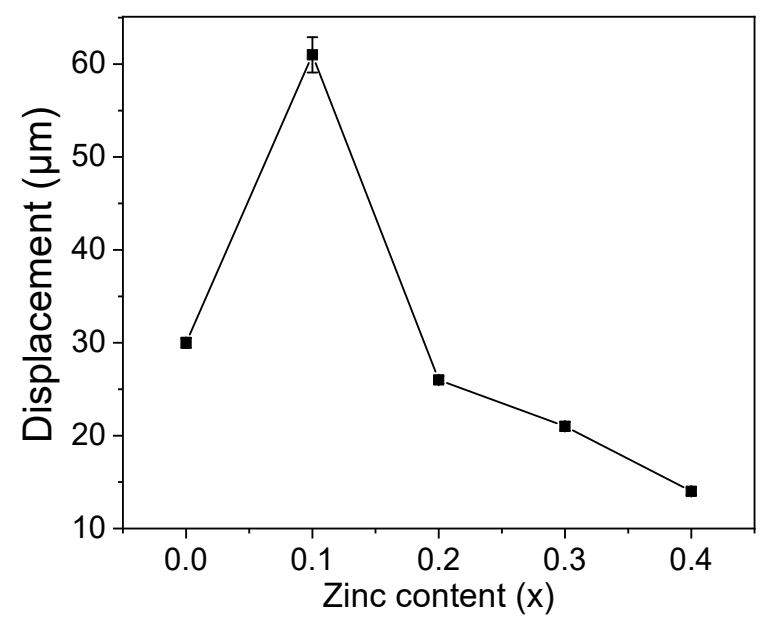

Figure 3.4 The observed displacement in gelatin phantoms for the phantoms containing inclusions filled with the nanoparticles prepared with different amounts of zinc.

As stated before, according to equation (3.5), the oscillation frequency of the nanoparticles due to produced magnetomotive force is twice that of the applied magnetic field. To confirm that 
the observed displacements were due to the interaction of magnetic field with nanoparticles, the power spectrum of the detected displacement within the inclusion, shown in figure 3.5, has a peak at a frequency two times greater than the applied magnetic field frequency $(50 \mathrm{~Hz})$, as expected [177].

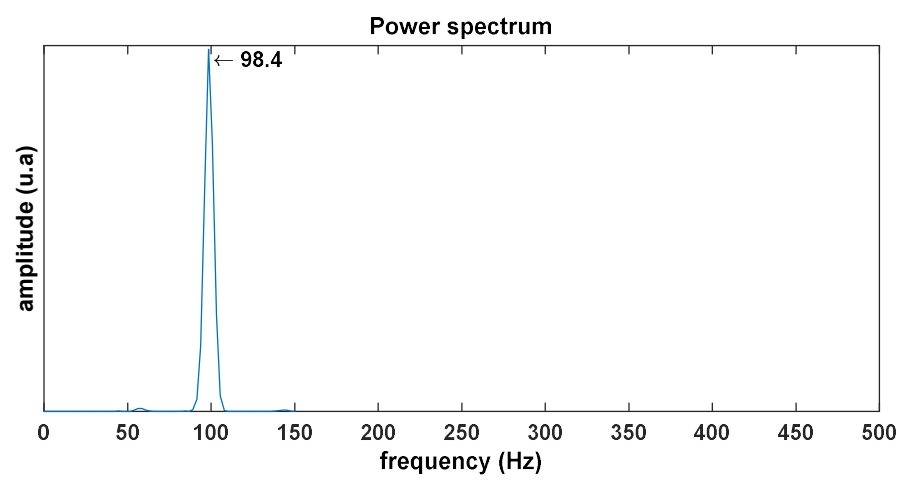

Figure 3.5 A typical power spectrum of the oscillation frequency resulting from the interaction of magnetic nanoparticles with the applied field

Finally, it is worth keeping in mind that although MMUS is considered a low cost imaging modality, the fact that magnetomotive force depends on magnetic field gradient and magnitude can lead to considerably higher costs when imaging higher depths or bigger volumes is the goal. Meanwhile, for applications such as phantom or small animals experiments, for example, it has several advantages such as capacity for real-time imaging, lower implementation cost and reduced system complexity [48].

\subsection{Summary}

Zinc substituted magnetite $\left(\mathrm{Zn}_{\mathrm{x}} \mathrm{Fe}_{1-\mathrm{x}} \mathrm{Fe}_{2} \mathrm{O}_{4} \mathrm{x}=0,0.1,0.2,0.3\right.$ and 0.4$)$ nanoparticles with superparamagnetic properties, which is a key factor in MMUS application, were successfully evaluated as contrast agent for MMUS. Sample $\mathrm{x}=0.1$ with enhanced saturation magnetization showed more than twofold increase in observed displacement compared to magnetite. The drastic increase in displacement for all magnetic nanoparticles in gelatin phantoms compared to the oil-based phantoms showed the crucial role of viscoelastic properties of the medium surrounding the nanoparticles. Since, it has been already demonstrated that [37] zinc substituted magnetite nanoparticles are nontoxic to healthy cells, this enhancement in magnetic properties of magnetite nanoparticles by zinc can open the possibility of its application in in vivo studies not only as diagnostic agent (contrast agent in medical imaging), but also as a therapeutic agent in magnetic hyperthermia simultaneously (theranostic agent). 


\section{Chapter 4 : A versatile induction heating system for magnetic hyperthermia studies under different experimental conditions}

\subsection{Introduction}

Cancer is one of the leading causes of death worldwide. Therefore, extensive research has focused on developing efficient methods for diagnosing and treating cancer. Especially, developing complementary therapeutic methods, e.g. hyperthermia, to increase the efficiency of surgery, radiotherapy or chemotherapy are in the center of attention. The medical use of heat to treat certain ailments can be traced back to the ancient epoch when Hippocrates (470-377 B.C.) believed that a patient who could not be cured by heat was actually incurable[189]. Today, hyperthermia, is considered as complementary to the aforementioned cancer therapy methods and refers to abnormal temperature rise in a part or the whole body above the normal temperature for a certain period of time[190]. Different hyperthermia techniques have been proposed such as microwaves (from 433 to $2450 \mathrm{MHz}$ ), radiofrequency electromagnetic fields (in the range of $100 \mathrm{kHz}$ to $150 \mathrm{MHz}$ ), infrared radiators, hot water perfusion, ultrasound, and resistive wire implants[191]. Recently [99, 192], magnetic hyperthermia using magnetic nanoparticles has found its way to the clinical trials as one of the promising methods for treating cancer.

In magnetic hyperthermia, magnetic nanoparticles accumulate within the tumor and subsequently a radiofrequency magnetic field is applied. Upon the application of this magnetic field, heat is generated through different mechanisms such as, Néel and Brownian relaxation[193]. The success of this technique depends on the combination of many factors such as adequate synthesis and characterization of the nanoparticles, biocompatibility and assessment of their performance under the operation conditions, efficient delivery to the target tissue, monitoring the temperature, and safety limitation of the applied magnetic field.

One of the most important properties of magnetic nanoparticle candidates for hyperthermia is their power absorption upon the application of the magnetic field. This is quantified by the specific absorption rate (SAR), which is defined as[194]: 


$$
\mathrm{SAR}=\frac{P}{m_{M N P}}
$$

where $P$ is the absorbed power and $m_{M N P}$ is the magnetic nanoparticles mass. Due to the strong dependence of SAR on the magnitude $\left(H_{0}\right)$ and frequency $(f)$ of the applied magnetic field, the accurate determination of SAR before planning the therapy requires instruments capable of generating a proper magnetic field with well-known parameters and spatial distribution. Despite the tremendous amount of research on synthesis and functionalization of magnetic nanoparticles for hyperthermia, which is growing exponentially every year[194], the progress and status of research on the design and development of instruments for magnetic hyperthermia is not growing at the same rate.

In recent years, several groups have reported custom made devices for magnetic hyperthermia. Lacroix et al. [195] built a system to generate magnetic field with the amplitude of $3.82 \mathrm{kAm}^{-}$ ${ }^{1}$ and frequency range $5-500 \mathrm{kHz}$ using an I-shaped $\mathrm{Ni}-\mathrm{Zn}$ ferrites with a gap of $1.1 \mathrm{~cm}$. Cano et al. [196] reported on a system based on resonant inverter with a 25 turn three layers solenoid coil with inner diameter of $5 \mathrm{~cm}$ generating a single frequency $(206 \mathrm{kHz})$ magnetic field of 12 $\mathrm{kAm}^{-1}$. Mazon et al.[197] to improve the system reported in Cano et al. [196] constructed a frequency variable system composed by a resonant inverter to generate the magnetic field over the frequency band $180-525 \mathrm{kHz}$ and field amplitude up to $30.2 \mathrm{kAm}^{-1}$. In another improvement, Mazon et al.[198] added a phase lock loop (PLL) circuit to their system to accurately tune the resonant frequency of the system during the experiments according to the inductance and capacitance of the resonant tank. Garaio et al. [199] described a multifrequency applicator (149-1030 kHz) consisted of an LCC resonant circuit driven by industrial linear amplifier. The work coil was a 9 turn solenoid with $25 \mathrm{~mm}$ diameter which could generate a homogeneous magnetic field with amplitude up to $35 \mathrm{kAm}^{-1}$, at low frequencies, within $15 \mathrm{~mm}$ diameter and $20 \mathrm{~mm}$ height cylindrical volume.

Bordelon et al. [200] designed and built a modified solenoid with two magnetic flux concentrators which significantly improved the homogeneity of the generated field. This coil was connected to an industrial induction power system which resulted in magnetic field with amplitude up to $50 \mathrm{kAm}^{-1}$ and frequency $150 \mathrm{kHz}$. Another example of alternating magnetic field with improved uniformity was reported by Barba et al. [201]. In their work, a system based on the Loney solenoid and correcting coils was used to generate uniform field insensitive to small variations of coil system parameters. Stauffer et al. [202] designed seven different 
coils, with dimensions to accommodate human body parts, to generate high magnetic fields driven with an industrial generator at fixed frequency of $100 \mathrm{kHz}$. These coils were designed to heat ferromagnetic seed implants located at different depths within the body. Although in these studies a wide range of field frequencies and amplitudes have been achieved, most of them suffer from either high costs because of utilizing commercial amplifiers or not discussing the flexibility in their coil design. The latter limits the application of the instrument in different conditions, e.g. liquid samples, in vitro or in vivo studies.

An induction heating system, in general, consists of an AC power supply to provide radiofrequency current and a resonant circuit. The current can be provided by either a function generator connected to an amplifier or a DC-AC inverter; and the resonant circuit can be in parallel or series format. Soft-switching is one of the most common and efficient techniques for DC-AC inverters [203]. For induction heating devices, e.g. magnetic hyperthermia, several different topologies using soft-switching technique have been proposed e.g. full-bridge[196], half-bridge[197], push-pull[204] etc. These topologies usually employ metal-oxide semiconductor field-effect transistor (MOSFET) device for switching. Push-pull inverters, due to their simple trigger circuit, high efficiency, being less affected by the input voltage ripple in current source configuration, and reduced number of switches for low to medium power applications, are one of the most favorable topologies [204-206].

Push-pull inverts have different derivatives which have been used in various applications such as power supplies for a cold-cathode fluorescent lamps[207], wireless powering and communication[208], flyback transformers[209, 210], high-voltage power supply for ozone generation[211], inductive welding[212], and induction heating[204]. Due to some drawbacks in this category of inverters and depending on each specific application, a variety of modifications have been proposed. In conventional Royer circuit[213], for example, highcurrent spike at the end of a transistor on-time significantly increases the transistor dissipation and may exceed the safe operating area and causing failure to the circuit[214]. Another example can be sever input voltage limitation in switches of cross-coupled oscillators[215] and high AC losses, which can destroy the switches in Mazzilli circuit. Mazzilli circuit is a derivative of the cross-coupled oscillator and Royer oscillator[215]. To overcome the aforementioned drawbacks, the authors in[215, 216] proposed two different gate drives for cross-coupled oscillator circuits to be used in high voltage converters. 
The circuit of the system proposed in the present paper is a modification of a Mazzilli crosscoupled oscillator similarly as described in $[215,216]$. In this setup, the resonant frequency of the tank circuit (regardless of its components) is directly followed, therefore, it can be used with different induction coil formats. In the present study, we report the design and construction of a versatile setup for magnetic hyperthermia studies. Heating curves obtained from the experiments conducted with the system using iron oxide nanoparticles are also shown. In fact, the main purpose of this study was to design a stable hyperthermia system which can operate under various experimental conditions.

\subsection{Materials and methods}

\subsubsection{The inverter circuit}

In a conventional Mazzilli inverter (figure 4.1(a)), the gates of the MOSFETs (Q1and Q2) are cross-coupled through a diode (D1 and D2) to the opposite drain and the resonant circuit directly controls the switches. This cross-coupled configuration through the diodes ensures the MOSFETs to not be turned ON simultaneously. In addition, these diodes are used to discharge the gate of the MOSFETs and to prevent the drain voltages from exceeding the maximum gatesource voltage. The modes of operation in this topology can be summarized as follow [212, $215,216]$ :

a) $\mathrm{Q}_{1}$ and $\mathrm{Q}_{2}$ have a duty cycle slightly more than $50 \%$ and the cross-coupling configuration assures they will not be turned ON simultaneously. Assuming $Q_{2}$ is turned OFF, $\mathrm{Q}_{1}$ turns $\mathrm{ON}$ and its drain provides the DC ground current return path. At this mode, the energy of the resonant circuit is stored in the inductor and the capacitor is completely discharged. Since switching of the MOSFETs occurs at zero-capacitor voltage, no resonant current passes through the switches.

b) While $\mathrm{Q}_{2}$ is turned $\mathrm{OFF}$ and $\mathrm{Q}_{1}$ is $\mathrm{ON}$, the resonant current proceeds and it is seen as the rise and fall of the capacitor's voltage. This current charges the capacitor to the maximum voltage (voltage reaches its peak at the drain of $Q_{2}$ ), storing all the energy in the capacitor. After reaching this maximum the voltage falls back to zero, transferring the whole energy to the inductor.

c) As the drain voltage in $\mathrm{Q}_{2}$ reaches to zero, $\mathrm{Q}_{2}$ turns $\mathrm{ON}$ and $\mathrm{Q}_{1}$ OFF. As in (a), the capacitor is completely discharged and the total current passes through the inductor but in the opposite direction.

d) Finally, the last mode is the rise and fall of the drain voltage of $\mathrm{Q}_{1}$ as in (b). 
Despite some advantages such as stability and self-tracking of the resonant frequency, this topology has some significant drawbacks when using high voltage and high frequencies. Since diodes are connected to the opposite drain, any small capacitance of this component can have a significant effect on the gate voltage, resulting in applying insufficient gate-source voltage during high-frequency operation. This can increase the switching losses to such a high level which destroys the switches [216]. Moreover, the gate drive power is supplied through a resistance (R1 and R2) and the gate capacitance is not negligible, resulting in an RC time constant and inhibiting fast switching at high frequencies [215]. To overcome these drawbacks and considering the magnetic hyperthermia application which implies higher frequencies $(>100$ $\mathrm{kHz}$ and $<1 \mathrm{MHz}$ ), the inverter was designed with an active gate drive which ensures fast switching and provides sufficient gate-source voltage. To maintain the self-oscillating feature of the circuit, the MOSFETs were cross-coupled through that driver.

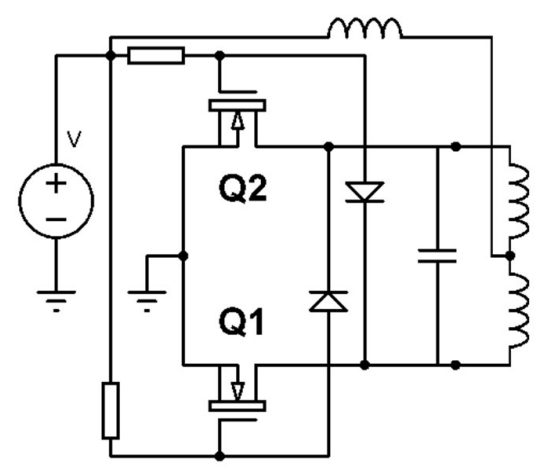

(a)

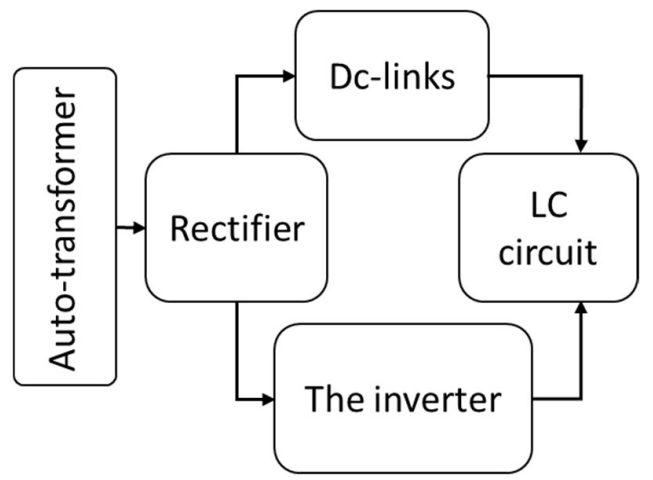

(b)

Figure 4.1 (a) A conventional Mazzilli circuit, (b) the block diagram of the proposed system.

Figure 4.1(b) illustrates the block diagram of the proposed system. It consists of a variable auto-transformer (AUJE, Industria Eletro Eletronica, Brasil, $2400 \mathrm{w}$ ) as power supply of the system, a rectifier circuit, a cross-coupled inverter with an active gate drive, dc-links, and finally a parallel resonant tank circuit which is quite flexible in its components, i.e. capacitors and the coil. Filters were included in the rectifier circuit to minimize the ripple effect in its output. The magnetic field in the coil is proportional to the AC current which is proportional to the output DC voltage of the rectifier. Hence, by varying the output voltage of the autotransformer, the magnetic field strength in the coil can be controlled. 


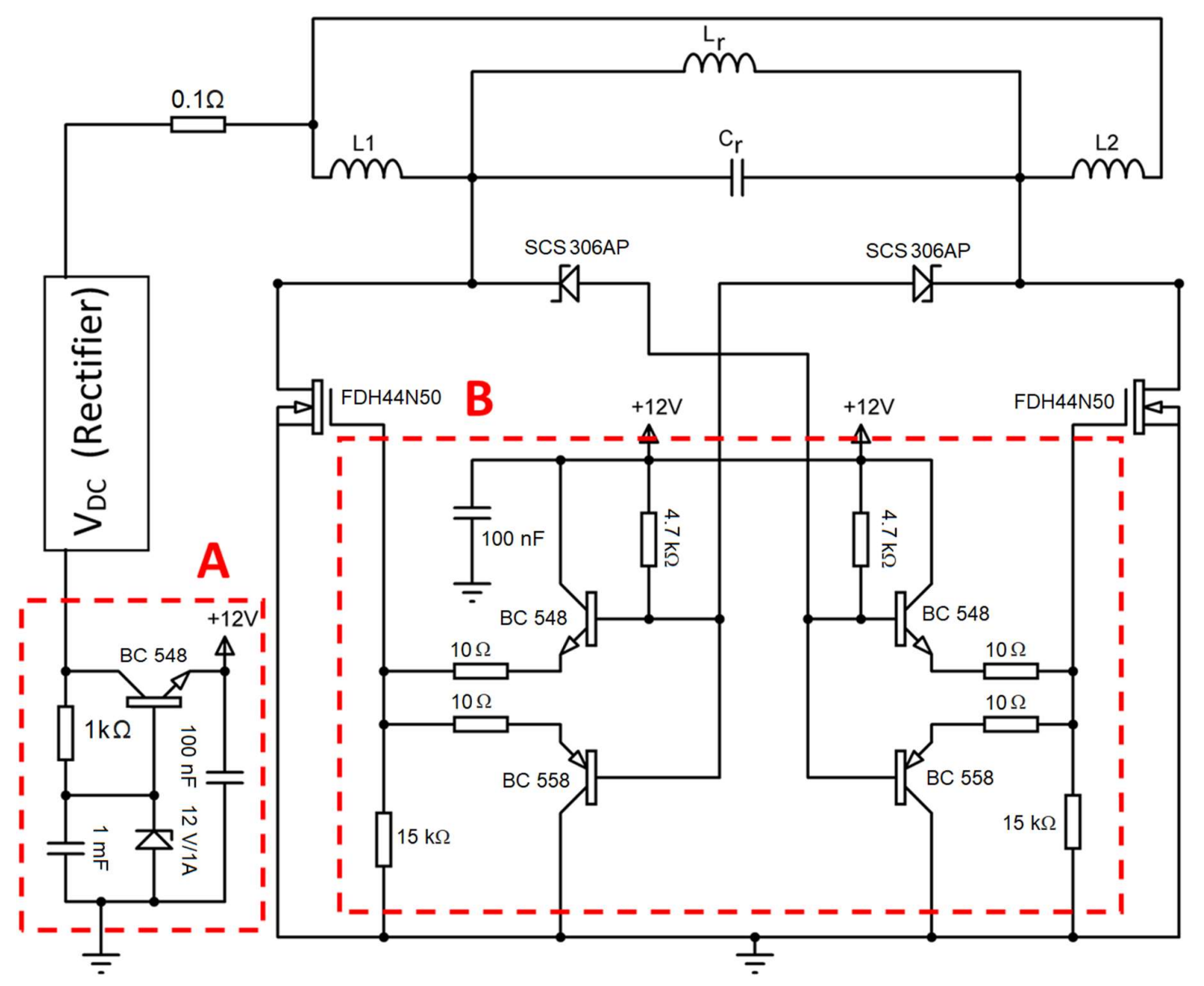

Figure 4.2 The complete diagram of the proposed circuit. In this circuit, A and B are the active gate drives.

Figure 4.2 shows the architecture of the designed inverter, which is a derivative of Mazzilli circuit but instead of supplying the gates through resistors (R1 and R2 in figure 4.1) an active gate drive ( $\mathrm{A}$ and $\mathrm{B})$ has been employed. This circuit has two dc-link inductors $\left(\mathrm{L}_{1}\right.$ and $\left.\mathrm{L}_{2}\right)$ to supply approximately a constant current to the inverter. This can be achieved because the inductances of $\mathrm{L}_{1}$ and $\mathrm{L}_{2}$ are significantly higher than $\mathrm{L}_{\mathrm{r}}$ (the inductor of the LC tank) [205, 217]. These inductors were made each with the resultant inductance of $\sim 90 \mu \mathrm{H}$. The advantages of this configuration are the lower voltage stress on the power switch and no highside driver circuits[218]. Two common-ground cross-coupled MOSFETs (Q1 and Q2) were utilized as power switches - each with a duty cycle that is slightly less than $50 \%$ to achieve zero-voltage switching and to prevent conducting of internal body diodes [217]. The crosscoupled nature of the inverter has been retained through diodes (D1 and D2); however, instead of supplying the gate drive through resistance, an active gate drive circuitry has been used. This active gate drive (the complete circuit is included as supporting information) was built using 
NPN-PNP bipolar junction transistors. This strategy prevents the RC time constant observed in the conventional Mazzilli inverter. The gate drive can sink and source the current of the MOSFETs gate at very high speeds in the order of few MHz [216, 219]. Moreover, it ensures the application of a sufficient and constant gate-source voltage to the MOSFETs (here in our system $12 \mathrm{~V}$ ) and hence preserving the self-oscillation feature of the inverter, which may not be achieved in conventional Mazzilli circuit due to the passive gate drive.

\subsubsection{LC tank description}

Since the inverter circuit has the self-tracking characteristic for resonant frequency of the LC tank, it can operate with a wide range of capacitance and inductance combinations. This provides a great flexibility for choosing the operation frequency as well as the coil format regarding different experimental setup. Therefore, four different coil sizes and formats were designed to demonstrate the flexibility of the proposed device for applications such as SAR determination of magnetic nanoparticles dispersed in liquids, solid media such as phantoms or small animal experiments, and in vitro hyperthermia experiments using cell culture plates. Two solenoids (S1 and S2), one Helmholtz-like (HL), and one pancake coil (P) were built using 3/16" ( $4.76 \mathrm{~mm}$ ) diameter copper refrigerator tubing isolated with heat-shrink tube (figure 4.3(a)). Therefore, all coils were closed wound as the final winding pitches were almost the same as the copper tube diameter. The HL coil can be considered as a solenoid with a gap in its center or two similar solenoids with common axis connected in series. Prior to wrapping the coils, the desired inductances were estimated using Coil32 software [220] and after wrapping they were measured using a KEYSIGHT E4980AL precision LCR meter. The dc-link inductors $\left(\mathrm{L}_{1}\right.$ and $\mathrm{L}_{2}$ ) were manufactured by wrapping two pulley shaped frames with copper cable of nominal cross section area $2.5 \mathrm{~mm}^{2}$. In order to create a variable capacitor, twelve metallized polypropylene film capacitors, each having the capacitance of $100 \mathrm{nF}$, and voltage withstand value of $2000 \mathrm{~V}$ were mounted on a copper-clad board such that the equivalent capacitance for the tank circuit could easily be changed.

Since push-pull inverters inherently follow the resonant frequency of the LC tank [213, 215$217,221,222]$, their resonant state can be considered as the resonant frequency of the LC tank. In an ideal condition, for the parallel tank circuit, the resonant frequency $\left(f_{\mathrm{r}}\right)$ is given by[223]:

$$
f_{r}=\frac{1}{2 \pi \sqrt{L_{r} C_{r}}}
$$


where $L_{r}$ and $C_{r}$ are the inductance and capacitance of the LC tank. A refrigeration system with operation temperatures from 0 to $100{ }^{\circ} \mathrm{C}$ was used as water cooling system for the coils. Using this system the coil temperature could be maintained at room temperature $\left(\sim \pm 0.5^{\circ} \mathrm{C}\right)$ upon passing the high amplitude and high frequency current. Moreover, the water circulation was designed such that it was also used for the MOSFET's heat sinks cooling.

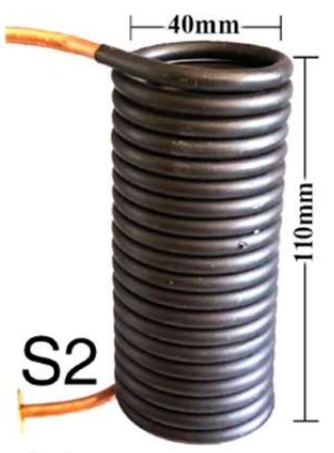

(a)

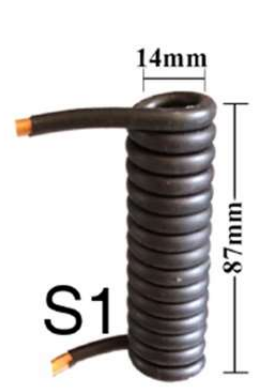

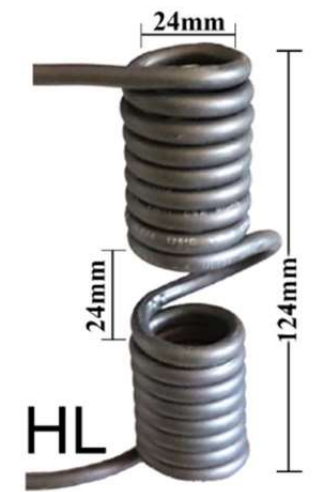

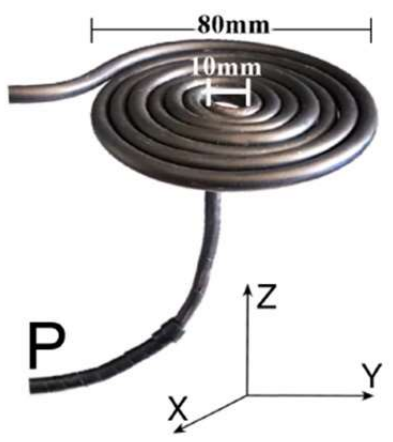

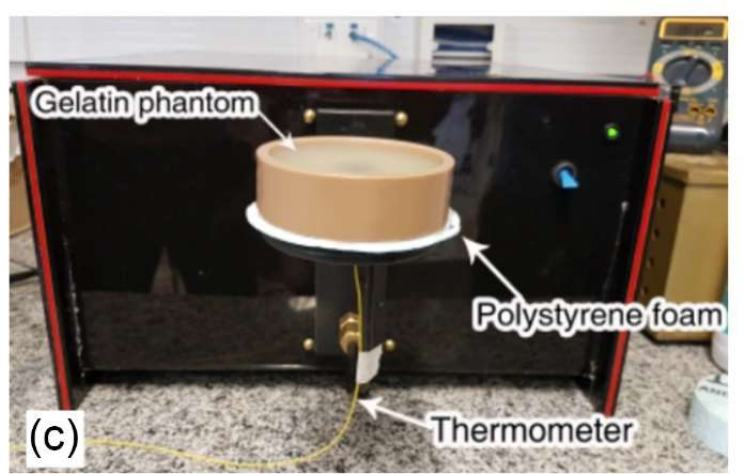

Figure 4.3 (a) Pictures of the designed coils, (b) the setup for the in vitro experiment with cell culture plates using the HL coil, (c) and the setup for gelatin phantom experiments using the P coil.

\subsubsection{Magnetic field measurement}

The experimental magnetic field amplitude for each coil were measured using the Faraday's law of induction. For this purpose, a one-turn pick up coil of $6 \mathrm{~mm}$ in diameter was wrapped around a polypropylene rod using copper wire AWG 25. Three axis linear translational stages were used to scan the regions of interests at $1 \mathrm{~mm}$ increments. The voltage amplitude $\left(\varepsilon_{0}\right)$, induced by the magnetic flux in the pickup coil, is related to the magnetic field amplitude as follow[224]:

$$
H_{0}=\frac{\varepsilon_{0}}{2 \pi \cdot f \cdot S \cdot \mu_{0}}
$$


where $S$ is the area of the pickup coil and $\mu_{0}$ the vacuum permeability. Experimental results were compared to simulations for S1, S2 and P coils. For this purpose, the current in each coil was estimated considering an ideal situation, where the surge impedance $\left(Z_{0}\right)$ of the tank circuit can be described as[225]:

$$
Z_{0}=\sqrt{\frac{L_{r}}{C_{r}}}
$$

and, therefore, the current as[196, 223]:

$$
I=\frac{V_{0}}{Z_{0}}=V_{0} \sqrt{\frac{C_{r}}{L_{r}}}
$$

where $V_{0}$ is the voltage amplitude across the coils. On-axis magnetic field strength due to the current $I$ in a finite solenoid coil wrapped with a thick conductor (here copper tube) is given by[226, 227]:

$$
H(z)=\frac{N I}{2 l\left(R_{2}-R_{1}\right)} \cdot\left(z \ln \frac{R_{2}+\sqrt{R_{2}^{2}+z^{2}}}{R_{1}+\sqrt{R_{1}^{2}+z^{2}}}+(l-z) \ln \frac{R_{2}+\sqrt{R_{2}^{2}+(l-z)^{2}}}{R_{1}+\sqrt{R_{1}^{2}+(l-z)^{2}}}\right)
$$

where $N$ is the number of the turns, $R_{1}$ is the inner and $R_{2}$ the outer diameters of the solenoid, $l$ the length and $z$ the distance from the center of the coil. Using equation (4.6) and the estimated current, the on-axis magnetic field of solenoid coils were theoretically calculated.

Minnaert et al.[228] calculated the z-direction magnetic field component $\left(H_{\mathrm{z}}\right)$ of a general planar spiral coil, in cylindrical coordinates system, at any point of space $\mathbf{r}(r, \varphi, z)$ using BiotSavart law. According to their work, knowing the function $r^{\prime}=f(\theta)$ describing the flat spiral shape conductor which carries the current $I$ between $\theta_{1}$ and $\theta_{2}$, its z-direction magnetic field component can be calculated by:

$$
H_{z}\left(r, r^{\prime}\right)=\frac{I}{4 \pi} \int_{\theta_{2}}^{\theta_{2}} \frac{\left[r \frac{d f(\theta)}{d \theta} \sin (\varphi-\theta)-r f(\theta) \cos (\varphi-\theta)+f^{2}(\theta)\right]}{\left[r^{2}+f^{2}(\theta)-2 r f(\theta) \cos (\varphi-\theta)+z^{2}\right]^{3 / 2}} d \theta
$$

Using numerical methods in MatLab software this component of the magnetic field for the $\mathrm{P}$ coil was simulated.

4.2.4. Magnetic nanoparticles synthesis and hyperthermia experiments 
To investigate the potential of the system for magnetic hyperthermia studies, iron oxide nanoparticles were synthesized using the coprecipitation method. Details about the preparation procedure has been reported elsewhere[90]. Prepared iron oxide nanoparticles were characterized by a powder X-ray diffraction using a Bruker Siemens AXS D5005 X-Ray diffractometer with $\mathrm{Cu} \mathrm{K} \alpha$ radiation $(\lambda=1.5406 \AA)$ and vibrating sample magnetometer (VSM) using Quantum Design MPMS ${ }^{\circledR}$ SQUID VSM DC magnetometer. Nanoparticles were dispersed in water and a suspension of $20 \mathrm{mg} / \mathrm{mL}$ was prepared to investigate their heat dissipation power due to the alternating magnetic field in liquid medium. The suspension at the concentration used in this study showed a good stability over weeks. Eppendorf tubes isolated with polystyrene foam were placed inside the solenoid coils and real-time temperature measurements were done using a fiber optic thermometer (Qualitrol NOMAD-Touch Portable Fiber Optic Monitor) with the accuracy of $\pm 0.1^{\circ} \mathrm{C}$. The experiment using the HL coil was done with the same concentration of magnetic nanoparticles using a 24 well cell culture plate (figure 4.3(b)).

In order to calculate the SAR values, first the experimental temperature data were fitted using the Box-Lucas function:

$$
T(t)=A(1-\exp (-B t))
$$

where the product of $A B$ stands for the initial slope of the heating curve[229]. Then SAR values were calculated as follow:

$$
\mathrm{SAR}=\left.\frac{C_{W} m_{W}+C_{n p} m_{n p}}{m_{n p}} \frac{\Delta T}{\Delta t}\right|_{t \rightarrow 0}
$$

where $m_{W}$ and $m_{n p}$ are the masses and $C_{W}$ and $C_{n p}$ are the specific heat capacity of water and nanoparticles, respectively.

A cylindrical tissue-mimicking gelatin phantom with $7.5 \mathrm{~cm}$ in diameter and $2.5 \mathrm{~cm}$ in height containing a cylindrical inclusion of magnetic nanoparticles were prepared to investigate the potential application of the $\mathrm{P}$ coil for in vivo experiments. The cylindrical inclusion with $2 \mathrm{~cm}$ in diameter and $1 \mathrm{~cm}$ in height consisted of $20 \mathrm{mg} / \mathrm{cm}^{3}$ nanoparticles concentration and was prepared with the same material used for the background of the phantom. The reader is referred to the reference [230] for details about the phantom preparation. The real-time temperature was recorded using the same thermometer by creating a narrow channel to place the tip of the optical fiber at the center of the inclusion. A $3 \mathrm{~mm}$ polystyrene foam was used on the surface of the $\mathrm{P}$ 
coil for thermal isolation (figure 4.3(c)). A control measurement was also conducted by placing the opposite side of the phantom on the surface of the P coil.

\subsection{Results and discussion}

To evaluate the circuit operation, the resonant frequency $63 \mathrm{kHz}$ was chosen to observe the waveforms at the steady state condition. Figure 4.4(a) shows the gate voltage signal across both $\mathrm{Q}_{1}$ and $\mathrm{Q}_{2}$ with 180 degrees phase difference, figure 4.4(b) shows the gate voltage across $\mathrm{Q}_{1}$ and drain voltage across $\mathrm{Q}_{2}$, and figure 4.4(c) shows the voltage across the resonant coil with its power spectrum. As it can be seen, these waveforms demonstrate that the expected performance and soft-switching of devices have been achieved.
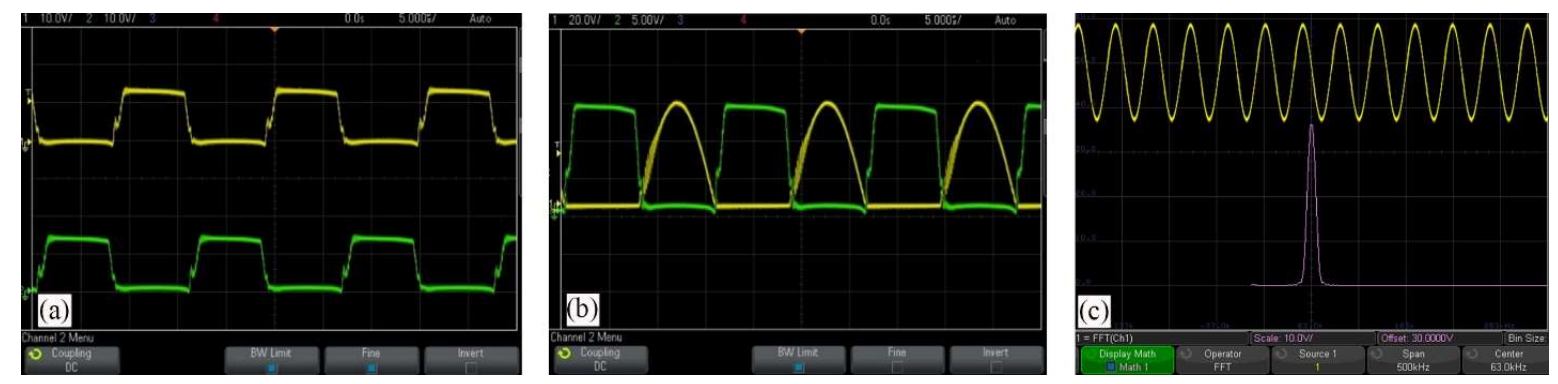

Figure 4.4 Voltage signal across (a) Q1 and Q2, (b) the gate of Q1 and drain of Q2, (c) and the resonant coil with its power spectrum.

Table 4.1 summarizes the physical characteristics of the designed coils. A variable capacitance for the resonant circuit was designed with twelve $100 \mathrm{nF}$ capacitors mounted on a copper-clad board and five different configurations were examined. The achieved frequencies and maximum field strength for each coil are presented in table 4.2. These values are in the range reports in literature $[82,231,232]$. Except for the $\mathrm{P}$ coil at higher frequencies, which the resultant magnetic field strengths were not usable, the observed magnetic field in the other coils offered a great flexibility in choosing a wide range of field parameters for different conditions. 
Table 4.1 The physical characteristics of the designed coils

\begin{tabular}{ccccc}
\hline Coil Format & Diameter $(\mathbf{m m})$ & Length $(\mathbf{m m})$ & $\begin{array}{c}\text { Number of } \\
\text { turns }\end{array}$ & Inductance $(\boldsymbol{\mu H})$ \\
\hline S1 & 14 & 87 & 15 & 1.1 \\
\hline S2 & 40 & 110 & 20 & 5.1 \\
\hline HL & 24 & 124 (the whole length) & 8 (each solenoid) & 1.9 \\
\hline P & 10 (inner) & 24 (gap's length) & 7 & 1.7 \\
\hline
\end{tabular}

Table 4.2 The measured frequencies $(\mathrm{kHz})$ and maximum field strength $(\mathrm{kA} / \mathrm{m})$ for different coils at five capacitance configurations.

\begin{tabular}{|c|c|c|c|c|c|c|c|c|c|c|}
\hline \multirow{2}{*}{$\begin{array}{c}\text { Coil } \\
\text { Format }\end{array}$} & \multicolumn{2}{|c|}{$75 \mathrm{nF}$} & \multicolumn{2}{|c|}{$99.99 \mathrm{nF}$} & \multicolumn{2}{|c|}{$200 \mathrm{nF}$} & \multicolumn{2}{|c|}{$400 \mathrm{nF}$} & \multicolumn{2}{|c|}{$1200 \mathrm{nF}$} \\
\hline & $f$ & $H_{0}$ & $f$ & $H_{0}$ & $f$ & $H_{0}$ & $f$ & $H_{0}$ & $f$ & $H$ \\
\hline S1 & 530 & $3.1 \pm 0.3$ & 475 & $3.6 \pm 0.5$ & 339 & $12.4 \pm 0.3$ & 240 & $13.2 \pm 0.2$ & 137 & $27.2 \pm 0.2$ \\
\hline S2 & 250 & $2.6 \pm 0.1$ & 219 & $3.4 \pm 0.1$ & 155 & $5.5 \pm 0.1$ & 110 & $9 \pm 0.2$ & 63 & $17 \pm 0.02$ \\
\hline $\mathbf{P}^{1}$ & - & - & - & - & 265 & $3.6 \pm 0.1$ & 187 & $7.1 \pm 0.2$ & 106 & $16.4 \pm 0.4$ \\
\hline$H_{L^{2}}$ & 408 & $1.2 \pm 0.2$ & 363 & $1.2 \pm 0.1$ & 258 & $2.4 \pm 0.1$ & 183 & $3.6 \pm 0.1$ & 105 & $11.1 \pm 0.1$ \\
\hline
\end{tabular}

The mean absolute difference between the measured frequencies and those predicted using equation (4.2) was below 3\%. For all 18 frequencies, the standard deviation calculated by considering 1000 frequency measurements, except for frequencies higher than $400 \mathrm{kHz}$, were below $1 \mathrm{kHz}$. The standard deviations were 10, 15, and $35 \mathrm{kHz}$ for frequencies 408, 475, and $530 \mathrm{kHz}$ respectively. Although this is strong evidence the system works very close to the resonant frequency, a rigorous confirmation about the resonant state can be achieved by monitoring the phase shift between the current and voltage in the LC tank. Moreover, the total harmonic distortion (THD) for all frequencies was below $1 \%$ except for the frequency $530 \mathrm{kHz}$ which was $2 \%$. These results show that the system proposed here successfully tracked the resonant frequency of its LC tank for frequencies below $400 \mathrm{kHz}$. The system also showed a limitation for maximum field strengths at higher frequencies (table 4.2). One possible solution to tune the resonant frequency can be conducted by implementing an appropriate phase lock loop circuit in the system $[198,204]$. However, for applications at frequencies lower than 400 
$\mathrm{kHz}$, the system proposed in this study can be satisfactory owing to its very simple structure and excellent flexibility in coil design for various experimental conditions. It is worth mentioning that in clinical magnetic hyperthermia routines, frequencies close to $100 \mathrm{kHz}$ are currently used [58].

Figures 4.5(a) and 4.5(b) show the simulated and measured on-axis magnetic field profiles of $\mathrm{S} 1$ and S2. The experimental measurements are reasonably in agreement with the theoretical calculations. The $\mathrm{S} 1$ coil, due to its geometry, provides almost constant field strength between $-22 \mathrm{~mm}$ to $20 \mathrm{~mm}$ considering the center of the coil on its axis as origin (volume of $6.8 \mathrm{~cm}^{3}$ ), which is highly suitable for SAR determination of liquid samples. It ensures the sample volume to be entirely immersed in a uniform magnetic field strength, therefore, improving the accuracy of SAR determination. The S2 coil, in addition to providing high magnitude and almost homogeneous magnetic field within a larger volume, it can offer different field frequency (almost the half of S1) with the same capacitors $\left(C_{r}\right)$ configuration.

The HL coil in this study was designed to provide a proper setup for in vitro magnetic hyperthermia experiments when using cell culture plates or strip [233, 234]. As can be seen in figure 4.3(b), this setup not only provides room for facile temperature measurements during the field application, but also it guarantees that only the magnetic nanoparticles in the desired well effectively sense the magnetic field. Meanwhile, the magnetic field profile of this coil shows that its solenoid shape parts can also provide a reasonable homogenous and high magnetic field. In order to have a better idea of the magnetic field in this coil, in addition to the on-axis field (figure 4.5(c)), its whole center volume was scanned using the pickup coil and the results are shown in figure $4.5(\mathrm{~d})$. 

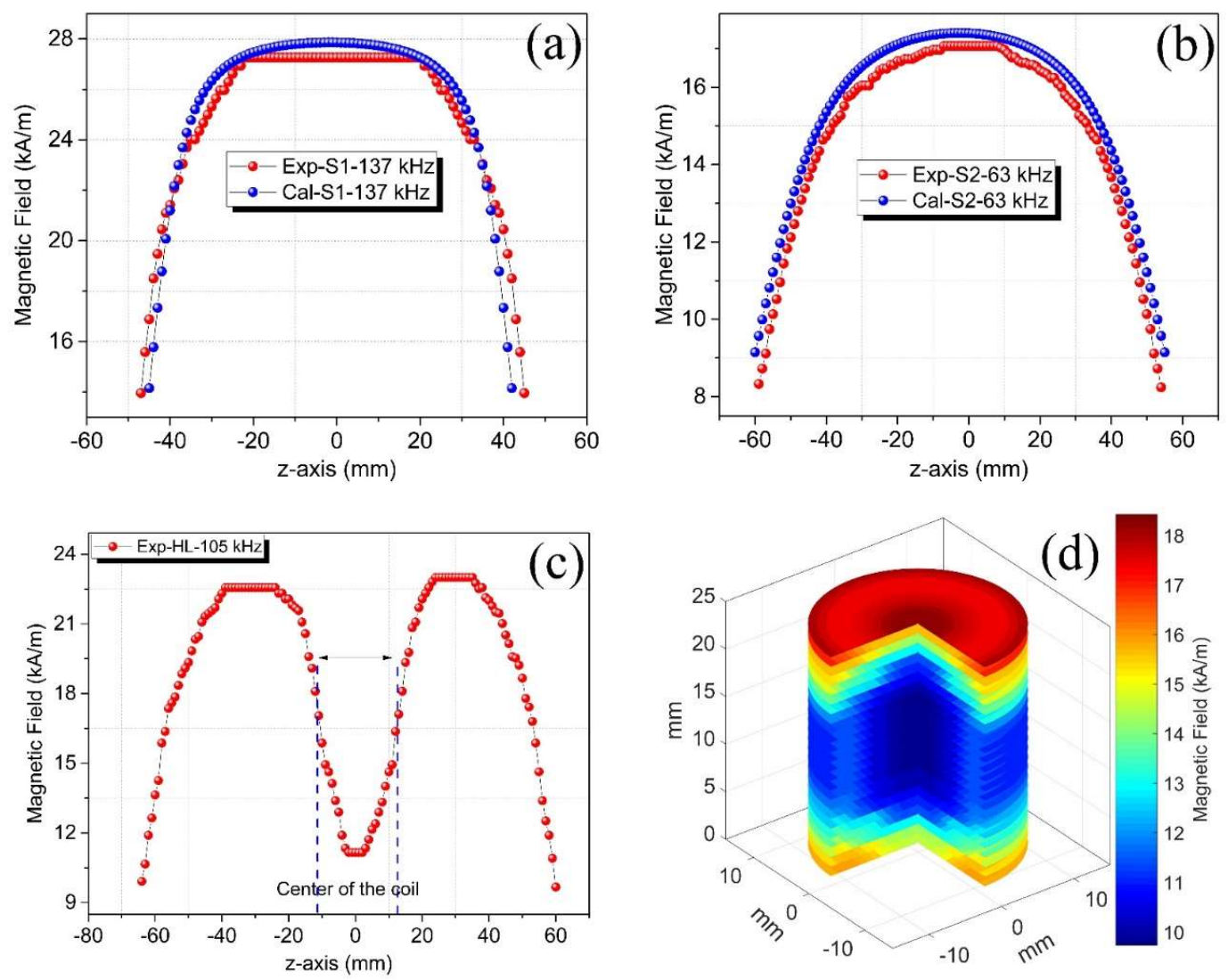

Figure 4.5 Simulated and measured on-axis magnetic field profiles of (a) S1 and (b) S2 coils, (c) measured onaxis and (d) 3D representation of the experimental magnetic field of HL coil.

Although solenoid coils are one of the most common formats to produce the field in magnetic hyperthermia studies [235], this format, due to its physical properties has a limitation to provide adequate space for in vivo or phantom experiments. Increasing the diameter of the coil not only changes its inductance significantly, but it also decreases the field amplitude providing that the same current can pass through the coil. Moreover, approaching the animal or gel phantoms for temperature measurement can also be another challenge with this kind of coils. Therefore, other coil formats such as pancake coil has always been considered as an alternative for solenoid coils [235-237]. The $\mathrm{P}$ coil designed in this study, at resonant frequency of $105 \mathrm{kHz}$ generated a relatively high magnetic field $(\sim 16.4 \mathrm{kA} / \mathrm{m})$ at the center of the parallel plane 1 millimeter from its surface. Figure 4.6(a) shows the measured magnetic field of this coil. Since this measurement was performed such that the surface of the pickup coil was always kept parallel to the surface of the coil, the resultant field profile is the $\mathrm{z}$ component of the magnetic field. 
Using numerical methods, this magnetic field component of the $\mathrm{P}$ coil was simulated (figure 4.6(b)) and the result confirmed the experimental measurements.
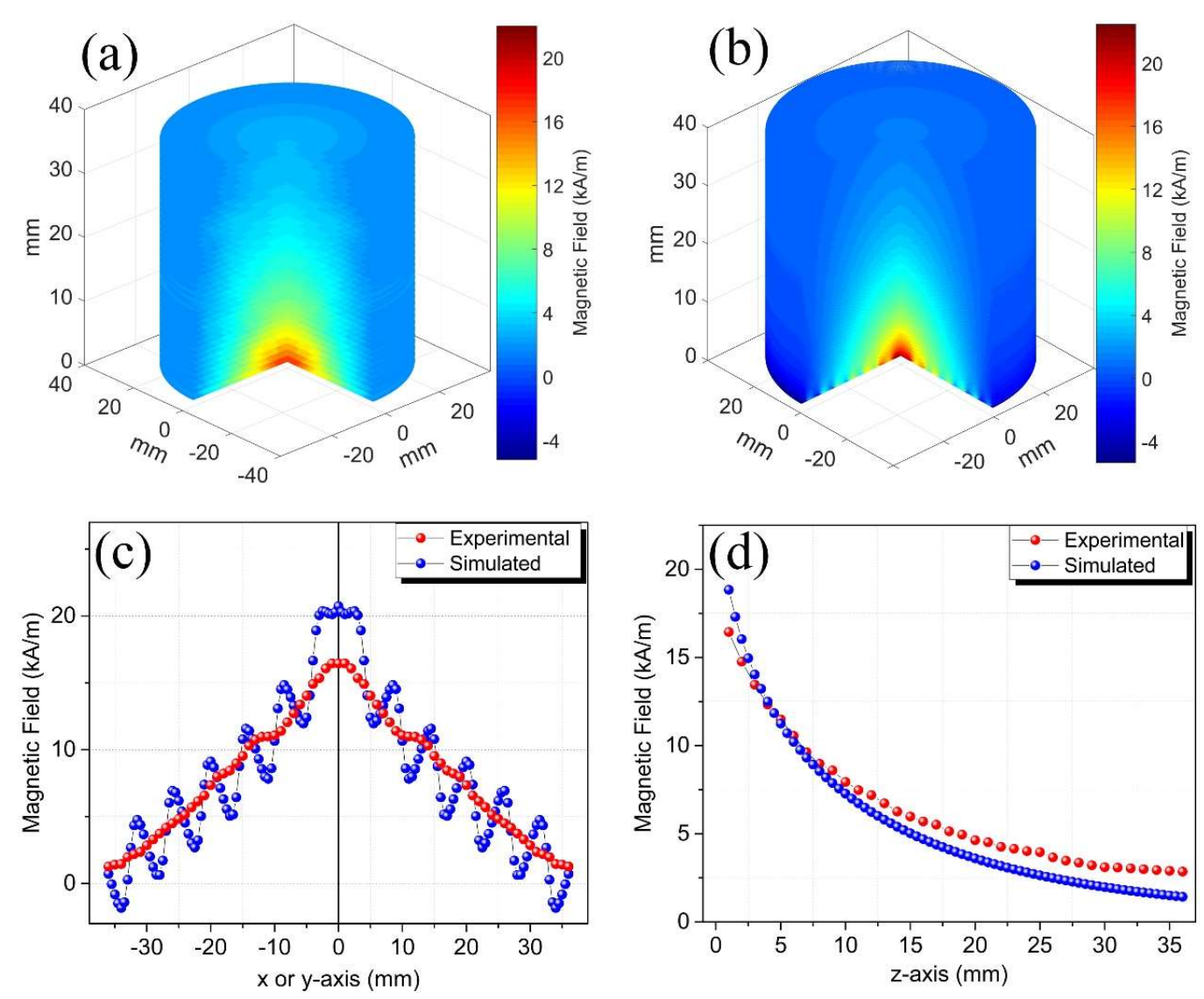

Figure 4.6 (a) Measured and (b) simulated z-component of the magnetic field of $\mathrm{P}$ coil. Measured and simulated magnetic fields of the $P$ coil: (c) $x$ or $y$-axis $1 \mathrm{~mm}$ away from its surface, and (d) z-axis.

When comparing the simulated with the experimental magnetic field results, it should be kept in mind that the currents used for simulations were obtained assuming ideal conditions. Especially at high frequencies, there will be an extra resistance in a conductor due to the skin effect[214]. At these frequencies, the current through a conductor mostly flows at the surface, resulting in a smaller cross section area and therefore a higher resistance. The distribution of the current falls from the surface of the conductor according to equation (4.10), where $J_{0}$ is the current density at the surface, $\delta$ is called "skin depth", $\mu$ and $\sigma$ are the permeability and the conductivity respectively [238]:

$$
J(x)=J_{0} \exp (-x / \delta)
$$




$$
\delta=\frac{1}{\sqrt{\pi f \mu \sigma}} .
$$

The extra resistance of a conductor, then, is given by[238]:

$$
R=\frac{1}{\sigma \delta}
$$

As an example, the AC/DC resistance ratio due to the skin effect in a copper wire AWG 18 at $200 \mathrm{kHz}$ is $\mathrm{R}_{\mathrm{AC}} / \mathrm{R}_{\mathrm{DC}}=1.85$ [214]. Therefore, all the calculated currents in this study are an approximation for simulating the magnetic profiles. In addition to these approximations in calculation of the current, in the case of $\mathrm{P}$ coil two more considerations should also be noted. First, for the simulation, the thickness of the conductor (copper tube) has not been taken into account. Second, in the experimental measurements the diameter of the pickup coil $(6 \mathrm{~mm})$ was larger than the copper tube $(\sim 4.76 \mathrm{~mm})$. Since in Faraday's law of induction the whole flux in the pickup coil is integrated, the changes in the magnetic field due to the transition between two adjusting turns will not be very clear as it is in the simulated results. Therefore, these can be the reasons for the observed difference in the simulated and measured magnetic field at a plan $1 \mathrm{~mm}$ away from the surface of the coil in figure 4.6(c).

To evaluate the system during magnetic hyperthermia studies under different experimental conditions, the prepared iron oxide nanoparticles were used. X-Ray diffraction patterns of the particles showed single phase spinel structure and their average crystallite size using Scherrer formula[118] was estimated to be $10 \mathrm{~nm}$. DC magnetic measurements (VSM) showed the nanoparticles are in superparamagnetic state with no coercivity and remanence. The saturation magnetization was $45 \mathrm{~A} . \mathrm{m}^{2} / \mathrm{kg}$. Figure 4.7(a) shows the temperature versus time curves obtained using the S1 coil at four different frequencies. Since the maximum field generated for this coil at the highest operation frequency $(530 \mathrm{kHz})$ was $3.1 \mathrm{kA} / \mathrm{m}$, this field amplitude was selected for the experiments. Figure 4.7(b) shows the results obtained with the other coils when the same capacitor configuration $(1200 \mathrm{nF})$ was chosen. In this case, the system was working with its maximum power for all coils. A 24 well cell culture plate was employed for the experiment using the HL coil and the experiment with the P coil was conducted using the gelatin tissue-mimicking phantom. In all cases a control experiment (sample containing only water) was conducted and no temperature rise was observed. In the case of the gelatin phantom using the $\mathrm{P}$ coil, the control measurement was conducted using a region of the phantom with no nanoparticles. Figure 4.7(c) shows the SAR values estimated for the curves shown in figure 4.7(a). 

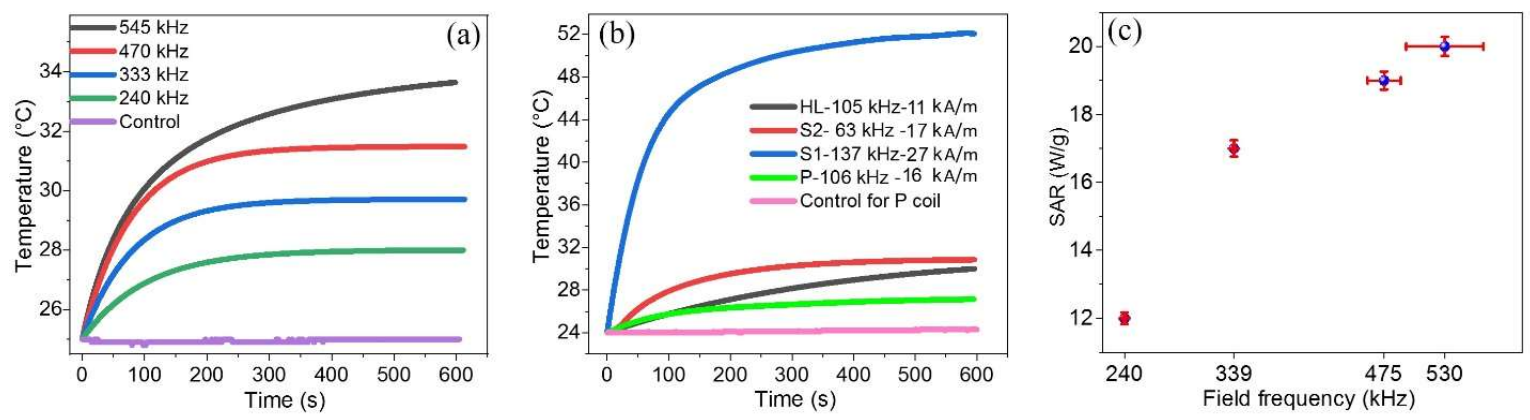

Figure 4.7 Temperature rise at different frequencies with a field amplitude of $3.1 \mathrm{kA} / \mathrm{m}$ using S1 coil, (b) temperature rise at maximum field strength of HL, S1, S2, and P coils using $1200 \mathrm{nF}$ capacitance for the tank circuit, and (c) the determined SAR values.

Different models for interpreting the experimental SAR values have been proposed [239-241]. At low field amplitudes the linear response theory can be used for this purpose. It has been proven that the criteria for the validity of this theory can be considered as $\mu_{0} H_{0} M_{s} V \leq k_{B} T$, where $V, \mathrm{k}_{\mathrm{B}}$, and $T$ are the magnetic particle volume, Boltzmann constant, and the absolute temperature, respectively [242]. In this framework, SAR values are directly related to the field frequency. Since it is not possible to compare the SAR values obtained from different experimental conditions, only the SAR values of the experiments using S1 coil were calculated. The results in figure 4.7(c) show that the SAR values increase with frequency. In figure 4.7(b), for example, the SAR value estimated for the experiment with the $\mathrm{S} 1$ coil operating at $137 \mathrm{kHz}$ was $66 \pm 1 \mathrm{~W} / \mathrm{g}$. This higher value was obtained because the field amplitude used was considerably higher that for all other frequencies. Meanwhile, when analyzing the experiments conducted with P and HL coils, one should notice the differences in experimental conditions. For example, the difference between the size of the sample holder when the experiment is conducted using the cell culture plate and a small eppendorf should be taken into account. In the case of the gelatin phantom experiment, in addition to the difference between the heat capacity of the medium in which nanoparticles are dispersed (gelatin phantom and water), Brownian relaxation mechanism is eliminated because of the immobility of the nanoparticles in the gel matrix.

Finally, it should be mentioned that the system proposed in this paper showed an excellent stability at all five tested capacitance configurations. For example, scanning the region of interest for measuring the magnetic field of the P coil, shown in figure 4.6(a), lasted around four hours and the system was continuously turned on at its maximum power during the measurement. However, the magnetic fields generated with the proposed system are only 
suitable for research purposes using liquid samples, in vitro, or in vivo studies with small animals. Generating appropriate magnetic fields for clinical applications, with larger coils, implies significant higher power consumption. In addition, a solenoid coil with $60 \mathrm{~cm}$ diameter and $30 \mathrm{~cm}$ length made with $1 \mathrm{~cm}$ diameter copper tube will lead to an inductance of ca. $5 \mathrm{mH}$, which is 1000 times higher than that of the S2 coil. This inductance would demand a new circuit design because: i) the dc-links inductors, L1 and L2 shown in figure 4.2, must have a

significantly higher inductance than the resonant coil; ii) the capacitance of the resonant tank would have to be drastically modified. Therefore, to consider this class of inverter for clinical magnetic hyperthermia applications, where extremely higher powers are demanded, further studies are necessary.

\subsection{Summary}

In this paper a low cost, frequency adjustable, and highly stable system was proposed for magnetic hyperthermia studies. The system showed great versatility to work under different experimental conditions, due to its flexibility in using different coil format designs. Five capacitance configurations and four coil formats in its LC tank circuit were examined and 18 different operation frequencies in the frequency range $63-530 \mathrm{kHz}$ with field strengths up to $27.2 \mathrm{kA} / \mathrm{m}$ were achieved. Since this system directly follows the resonant frequency of its LC tank circuit, it can potentially be used with other capacitor configurations and coil formats. To evaluate the proposed system, water dispersed iron oxide nanoparticles were synthesized with the coprecipitation method and were tested successfully for magnetic hyperthermia experiments under different experimental conditions. In summary, the results presented here showed that modifications in the Mazzilli inverter, which is mostly used in applications such as converters, resulted in a low cost and stable option for custom made hyperthermia device. 


\section{Chapter 5 : Role of zinc substitution in magnetic hyperthermia properties of magnetite nanoparticles: interplay between intrinsic properties and dipolar interactions}

\subsection{Introduction}

It was estimated that 9.6 million people worldwide would die from cancer in 2018 and this number is projected to reach over 13 million in 2030 [3, 4]. Early and accurate diagnosis and effective therapy are key factors for increasing the survival rate. Therefore, in recent decades tremendous efforts were focused on the discovery and development of new diagnostic and therapeutic methods. Magnetic hyperthermia using magnetic nanoparticles has received special attention as a promising therapeutic technique to combat cancer. It aims to selectively raise the temperature of cancerous cells to induce apoptosis and/or necrosis. In addition, hyperthermia can be used to increase the efficiency of conventional therapeutic procedures such as radiotherapy and chemotherapy [54].

The power dissipated by magnetic nanoparticles exposed to an alternating magnetic field with frequency $f$ and amplitude $H_{0}$ is converted into heat. This dissipated power $(P)$ for one cycle of magnetic field, which is related to the lag between the magnetic response of the material and the external magnetic field, can be expressed as [193, 242]:

$$
P=\int_{-H_{0}}^{+H_{0}} \mu_{0} M(H) d H
$$

where $\mu_{0}$ is the permeability of the free space and $M(H)$ the magnetization of the material. Accordingly, heating efficiency of the magnetic material, typically referred to as specific loss power (SLP), is defined as $\mathrm{SLP}=P f / \rho$; where $\rho$ is the density of the magnetic nanoparticles. Besides the dependence on intrinsic physical properties of the magnetic nanoparticles, SLP is directly proportional to the frequency and amplitude of the magnetic field. However, due to the non-specific heating arising from the induced eddy current in tissues, there is a restriction limit for the frequency and amplitude of applied field. This limit, which is the threshold for major discomfort in the patient and depends on the body area exposed to the magnetic field, was 
originally [243] defined as $H_{0} f \leq 4.85 \times 10^{8} \mathrm{~A} \cdot \mathrm{m}^{-1} \mathrm{~s}^{-1}$. Later, Hergt et al. [244] for smaller radii body parts estimated this limit as $H_{0} f \leq 5 \times 10^{9} \mathrm{~A} \cdot \mathrm{m}^{-1} \cdot \mathrm{s}^{-1}$. Therefore, optimizing the intrinsic properties of the magnetic nanoparticles to enhance their SLP, respecting this restriction for applied magnetic field, is of considerable importance.

In addition to the applied magnetic field parameters and the intrinsic properties of the magnetic nanoparticles, dipolar interactions also can play an important role in magnetic hyperthermia. Since, in a real colloidal dispersion of magnetic nanoparticles, in addition to the existence of particle aggregation there may always exist a degree of dipolar interactions even in diluted samples $[245,246]$. This dipolar interactions can sometimes modify the heating efficiency of the system up to two orders of magnitude [245]. Recently, a large number of studies have dealt with the effect of interparticle interactions and apparently a wide range of different theoretical and experimental results have been observed. For example, increase [247-250], decrease [251$254]$, and non-monotonic variation $[246,255,256]$ in SLP due to the dipolar interaction have been reported. Even constant SLP with sample concentration, which is one of the key factor governing the interparticle interactions, has been observed [257]. In several studies, these results have been interpreted based on morphological parameters such as the shape and size of aggregates, clusters, and chained formed in the colloids [258, 259], while it has also been shown that the intrinsic magnetic properties of the nanoparticles such as saturation magnetization or anisotropy are key factors in determining how dipolar interaction can affect the heating efficiency $[246,255,260]$. Therefore, to optimize the heating efficiency of the magnetic nanoparticles a great knowledge of their intrinsic and extrinsic properties is crucial.

Among different magnetic materials considered to be used in biomedical applications including magnetic hyperthermia, spinel ferrite nanoparticles, in particular magnetite and maghemite, are the most popular materials $[261,262]$. To date, much effort has been devoted to tune their intrinsic properties using different strategies such as modifying the synthesis procedures [134, $261,263]$ or doping various transition metal cations in their structure [37, 102, 264]. Of particular interest is to substitute small amounts of $\mathrm{Zn}^{2+}$ for divalent transition metals in the structure of this class of magnetic materials [37, 64, 66, 90, 265, 266]. Zinc is a diamagnetic cation with zero magnetic moment and has a strong affinity to occupy tetrahedral sites in spinel structure [18]. In magnetite, for example, this weakens the antiferromagnetic coupling between iron cations distributed on both tetrahedral and octahedral sites; therefore, resulting in higher saturation magnetization of the composition. However, by further increase of zinc content, 
exchange interactions within the octahedral sites would be dominant, leading to spin canting and a decrease in the magnetization of the system $[90,120]$.

Another interesting feature of doping zinc in ferrite nanoparticles is its effect on nanoparticles' structure and morphology. It has been shown in several studies that by increase zinc content in ferrites, nanoparticle sizes decrease systematically due to obstructing the crystal growth in spinel structure. On the other hand the lattice parameter increases by addition of zinc, mostly due to the site occupancy preference and the difference between the ionic radius of zinc and $\mathrm{Fe}^{3+}$ cations in tetrahedral sites $[90,115,117]$. These changes can, consequently, affect other characteristics of the host nanoparticles such as Curie temperature, blocking temperature, and anisotropy constant $[90,111,267]$. Therefore, zinc has always been regarded as a promising candidate to tune the magnetic properties of ferrite nanoparticles for biomedical applications, especially for magnetic hyperthermia [37, 64, 66, 121, 262, 266-270].

In our previous study [90] we reported on the synthesis and characterization of zinc-substituted magnetite nanoparticles $\left(\mathrm{Zn}_{\mathrm{x}} \mathrm{Fe}_{1-\mathrm{x}} \mathrm{Fe}_{2} \mathrm{O}_{4}, \mathrm{x}=0.0,0.1,0.2,0.3\right.$ and 0.4$)$ as contrast agents in magnetomotive ultrasound imaging. Improving the saturation magnetization, achieved by substitution of zinc in magnetite structure, for this imaging technique played a key role to improve the signal to noise ratio of the images. Addition of zinc in magnetite structure using a simple synthesis method not only modified the saturation magnetization, but also yielded to tailoring other intrinsic properties such as particles' size and anisotropy; which are all deterministic parameters for magnetic hyperthermia. This can in turn provide a great opportunity to explore the impact of each parameter on the magnetic hyperthermia property of the compositions. In the present paper, we aimed to study the magnetic hyperthermia response of these compositions at a wide range of experimental conditions. Moreover, the effect of dipolar interaction, which strongly depends on intrinsic properties of the particles, was investigated by changing the concentrations of the samples. Finally, the SLP values in the framework of the linear response theory were numerically simulated to infer the effect of dipolar interactions results.

\subsection{Materials and Methods}

\subsubsection{Theory}

Successful analytical description of power dissipation, considering a dispersion of noninteracting nanoparticles with randomly oriented magnetic moments, has been developed in linear regime wherein the condition $\mu_{0} H_{0} M_{s} V \leq k_{B} T$ is satisfied and the magnetization is linearly 
related to the applied magnetic field $(M=\chi H)[239,242,271]$. Here, $M_{s}$ is the saturation magnetization, $V$ the volume and $\chi=\chi^{\prime}-i \chi^{\prime \prime}$ the complex susceptibility of the nanoparticles, $k_{B}$ the Boltzmann constant, and $T$ the absolute temperature. Indeed, $\chi^{\prime}$ is the in-phase and $\chi^{\prime \prime}$ the out-of-phase component of the susceptibility also known as the loss component. In this framework, dissipated power is described as:

$$
P=\pi \mu_{0} H_{0}^{2} f \chi^{\prime \prime} \quad \text { where } \quad \chi^{\prime \prime}=\frac{2 \pi f \tau}{1+(2 \pi f \tau)^{2}} \chi_{0},
$$

In equation (5.2), $\chi_{0}$ is the static susceptibility and can be defined as $\mu_{0} M_{s}^{2} V / 3 k_{B} T, \tau$ is the effective relaxation time given by $\tau=\tau_{N} \tau_{B} /\left(\tau_{N}+\tau_{B}\right)$, in which $\tau_{N}$ and $\tau_{B}$ are the Néel and Brownian relaxation times, respectively, and defined as [242]:

$$
\begin{gathered}
\tau_{N}=\tau_{0} \exp \left(E_{A} / k_{B} T\right), \\
\tau_{B}=3 \eta V_{h} / k_{B} T,
\end{gathered}
$$

where $\tau_{0}$ is the inverse attempt frequency which depends on a variety of intrinsic properties such as saturation magnetization and magnetic anisotropy and can have values between $10^{-13}$ $10^{-8} \mathrm{~s}[251,272] . E_{A}=K_{\text {eff }} V$ is the energy required to flip the magnetic moment of the particle or the activation energy barrier which separates two opposite directions of magnetic moment in a single domain particle with an uniaxial anisotropy. Here, $K_{\text {eff }}$ is the effective anisotropy which can have different contributions from sources such as magnetocrystalline and surface anisotropy. In equation (5.4), $\eta$ is the viscosity of the medium and $V_{h}$ the hydrodynamic volume of the nanoparticles. Finally, SLP can be calculated using equation (5.2) as SLP $=P / \rho$. Numerical simulations of SLP in the framework of the linear response theory were conducted to aid interpretation of experimental data. Variation of the SLP values with respect to the dimensionless parameter $\sigma=K_{\text {eff }} V / k_{B} T$ was investigated to understand the heating efficiency of the samples.

Moreover, the dynamic hysteresis loop of the magnetic nanoparticles in an oscillating magnetic field $H(t)=H_{0} \cos (2 \pi f)$ was also calculated by [242]:

$$
M(t)=\frac{\chi_{0}}{\left(1+(2 \pi f \tau)^{2}\right)^{1 / 2}} H_{0} \cos (2 \pi f t+\varphi),
$$

where $\varphi=\arctan (2 \pi f \tau)$ is the phase lag between the magnetization and the external field. 


\subsubsection{Magnetic nanoparticles}

Zinc-substituted magnetite nanoparticles $\left(\mathrm{Zn}_{\mathrm{x}} \mathrm{Fe}_{1-\mathrm{x}} \mathrm{Fe}_{2} \mathrm{O}_{4}, \mathrm{x}=0.0,0.1,0.2,0.3\right.$ and 0.4) were prepared using the coprecipitation method. Their sizes were determined by transmission electron microscopy, TEM, (JEOL JEM- 100 CXII instrument) and vibrating sample magnetometer (QUANTUM DESIGN MPMS® SQUID VSM DC magnetometer) was used to characterize their magnetic properties. Details about synthesis and characterization of the magnetic nanoparticles are fully presented in [90]. The hydrodynamic size and the zetapotential of the particles was measured by dynamic light scattering (DLS) using a Zeta-Sizer system (Malvern Instruments). The Brownian relaxation times were calculated using the obtained sizes from DLS measurements and equation (5.4). In order to estimate the effective anisotropy constant of the samples, at sufficiently high magnetic fields, we fitted the magnetization curves using the law of approach to saturation [273] as:

$$
M=M_{s}\left(1-\frac{a}{H}-\frac{b}{H^{2}}\right)+c H,
$$

where $b=\beta K_{\text {eff }}^{2} / \mu_{0}^{2} M_{s}^{2}$ and for magnetic nanoparticles with uniaxial anisotropy, $\beta$ is $4 / 15$ [274]. Then, the Néel relaxation time of each sample was calculated using equation (5.3).

\subsubsection{Magnetic hyperthermia experiments}

The magnetic field was generated using a homemade frequency adjustable magnetic hyperthermia system. A solenoid coil (14 $\mathrm{mm}$ in diameter and $87 \mathrm{~mm}$ in height) capable of generating a homogenous field in the volume of $6.8 \mathrm{~cm}^{3}$, ensuring the whole sample is immersed in a region with uniform amplitude, was used.

For the calorimetric measurements of SLPs, we, initially, prepared aqueous dispersions of the nanoparticles with concentration $c=3.5 \mathrm{wt} \%$. Then, by successive dilutions, lower concentrations including $c=3.5,2.5,1.5,0.75,0.3$ and 0.03 wt.\% were obtained. All samples, at these concentrations and $\mathrm{pH} 6$, showed a good colloidal stability over several weeks. All the hyperthermia measurements were performed under as similar as possible conditions, i.e. the sample holder (eppendorf vial) and its volume $(300 \mu \mathrm{L})$, its position within the magnetic field, and measurement time $(600 \mathrm{~s})$. Three field frequencies $(f=137,240,339 \mathrm{kHz})$ with amplitude $H_{0}=7.5 \mathrm{kA} / \mathrm{m}$ were chosen in addition to a field dependence study at $339 \mathrm{kHz}$. Field parameters in all experiments were under the criteria of linear response theory and also satisfying the safety restriction introduced by Hergt et al. [271]. 
A fiber optic thermometer system (Qualitrol NOMAD-Touch Portable Fiber Optic Monitor) was used to record the temperature of the samples with accuracy of $\pm 0.1^{\circ} \mathrm{C}$. To calculate the SLP values, the temperature versus time results were fitted using the Box-Lucas equation as:

$$
T(t)=A(1-\exp (-B t))
$$

where the product $A B$ stands for the initial slop of the heating curves [229]. This is considered as one of the most accurate methods to calculate the SLP values for non-adiabatic experimental conditions [275]. Then the SLP values were calculated as:

$$
\mathrm{SLP}=\left.\frac{C_{W} m_{W}+C_{n p} m_{n p}}{m_{n p}} \frac{\Delta T}{\Delta t}\right|_{t \rightarrow 0},
$$

where $m_{W}$ and $m_{n p}$ are the masses and $C_{W}$ and $C_{n p}$ are the specific heat capacity of water and nanoparticles, respectively. Although our experimental setup was non-adiabatic, by using sufficient insulators and water cooling of the coil, the temperature of the coil could be controlled with accuracy $\pm 0.5^{\circ} \mathrm{C}$; therefore, any evidence of peripheral heating caused by the coil was eliminated. This was tested using a blank sample (pure water in the same holder) and no temperature change was observed.

\subsection{Results}

\subsubsection{Experimental results}

The characteristics of the samples are listed in table 5.1. The mean particle diameter $(<d>$ ) from TEM images (results published in a previous study [90]) and the hydrodynamic sizes $\left(D_{\mathrm{h}}\right)$ from DLS measurements show a relatively narrow size distribution. The sizes measured by DLS are larger than those measured by TEM. This difference in the mean sizes is expected due to both the hydration and the surface charge of the samples [276, 277]. The zeta potential of the samples $x=0.0,0.1,0.2,0.3$, and 0.4 were 16.2 (5.9), 25.6 (3.4), 15.1 (5.9) 20.3 (5.1), and 24.7 (3.5) $\mathrm{mV}$, respectively, where the numbers in parentheses are the zeta deviations. The zeta potential close to $20 \mathrm{mV}$ indicates a relatively good colloidal stability for all samples, therefore, the larger size of the particles measured by DLS can be related to the hydration of the samples.

The calculated effective anisotropy constants utilizing the law of approach to saturation and relaxation times of each sample are summarized in table 5.1. Equation (5.6) fitted all experimental data with R-squared values higher than $98 \%$. Since the magnetization curves for samples x $=0.2,0.3$, and 0.4 were not completely saturated, $M_{s}$ for these samples were estimated by plotting the high field values of $M$ versus $1 / H$ and the intercept of the line with the $M$ axis 
$(H=\infty)$ were chosen as $M_{s}$. This behavior can be an indication for the presence of a higher anisotropy in these samples [139]. The obtained $K_{\text {eff }}$ for sample x=0 is slightly higher than that for bulk magnetite, $18 \mathrm{~kJ} / \mathrm{m}^{3}$ [278], but very close to the other reports with the same particle size and prepared with coprecipitation method [279-281]. However, one should keep in mind that due to instability of the magnetite nanoparticles, especially prepared by coprecipitation method, there always exists a degree of oxidation in the surface of the particles, as it may form a core-shell composition of magnetite-maghemite [282, 283]. Park et al. [284] also observed a partial oxidation in magnetite nanoparticles of $12 \mathrm{~nm}$ prepared with thermal decomposition method and the estimated anisotropy were very close to ours. Recently, Modaresi et al. [285] reported on the effect of zinc doping level on magnetic properties of ferrite nanoparticles $\left(\mathrm{Zn}_{\mathrm{x}} \mathrm{Fe}_{3-\mathrm{x}} \mathrm{O}_{4}\right)$ prepared by coprecipitation method. In their study the effective anisotropy was also estimated using the law of approach and the result, contrary to ours, showed a direct relation between particle size and the effective anisotropy. However, all particle sizes in their study were considerably larger than the present study. For example, for sample $x=0.3$ with particle size $\sim 37 \mathrm{~nm}$ they estimated the effective anisotropy as $13.7 \mathrm{~kJ} / \mathrm{m}^{3}\left(56 \mathrm{~kJ} / \mathrm{m}^{3}\right.$ in our results). Although the addition of zinc up to certain amounts in ferrites can result in a decrease in their effective anisotropy [19], here, the effective anisotropy was increased by increasing the zinc content which is most probably due to the size effects [286]. Upon decreasing the nanoparticles size, here induced by increasing the zinc content, the surface effects become progressively dominant. Disorder in lattice symmetry close to and at the surface appears and disordered spins at the surface induce additional anisotropy [287, 288]. It should also be mentioned that in an assembly of magnetic nanoparticles with certain size distribution, a distribution of anisotropy constants exists [280]. However, we considered the obtained values as the mean values for $K_{\text {eff }}$ in all calculations.

Table 5.1 Characteristics of the samples, the numbers in parentheses for $\langle d\rangle$ are the polydispersity degree calculated as (standard deviation/mean particle diameter) and for $D_{\mathrm{h}}$ are the polydispersity index (PDI) provided by the zeta-sizer instrument.

\begin{tabular}{cccccccc}
\hline Sample & $\mathrm{M}_{\mathrm{s}}(\mathrm{kA} / \mathrm{M})^{[\mathbf{9 0}]}$ & $<d>(\mathrm{nm})^{[\mathbf{9 0}]}$ & $D_{\mathrm{h}}(\mathrm{nm})$ & $K_{\text {eff }}\left(\mathrm{kJ} / \mathrm{m}^{3}\right)$ & $\sigma$ & $\tau_{N}(\mathrm{~s})$ & $\tau_{B}(\mathrm{~s})$ \\
\hline $\mathrm{x}=0$ & 270 & $13.7(0.15)$ & $85(0.24)$ & $23 \pm 2$ & 7.4 & $1.6 \mathrm{E}-06$ & $2.3 \mathrm{E}-04$ \\
$\mathrm{x}=0.1$ & 425 & $12.4(0.12)$ & $75(0.11)$ & $27 \pm 3$ & 6.7 & $8.5 \mathrm{E}-07$ & $1.6 \mathrm{E}-04$ \\
$\mathrm{x}=0.2$ & 291 & $9.6(0.12)$ & $75(0.13)$ & $43 \pm 6$ & 4.9 & $1.3 \mathrm{E}-07$ & $1.61 \mathrm{E}-04$ \\
$\mathrm{x}=0.3$ & 255 & $6.5(0.16)$ & $40(0.32)$ & $56 \pm 7$ & 2 & $7.2 \mathrm{E}-09$ & $2.4 \mathrm{E}-05$ \\
$\mathrm{x}=0.4$ & 251 & $5.3(0.15)$ & $55(0.41)$ & $65 \pm 9$ & 1.2 & $3.4 \mathrm{E}-09$ & $6.3 \mathrm{E}-05$ \\
\hline
\end{tabular}


The heating efficiency of the samples was evaluated as a function of frequency and amplitude of the applied magnetic field as well as sample concentration. The latter determines interparticle distances in the colloidal system which in turn can affect the interparticle interactions. Figure 5.1 shows the concentration dependence of SLP at frequencies 137, 240, and $339 \mathrm{kHz}$. Although each sample at different frequencies showed similar trends by varying its concentration, we found significant different behaviors by changing the concentration of each sample for all frequencies. Samples $\mathrm{x}=0.2,0.3$, and 0.4 showed almost no concentration dependence, while the SLP values for samples $\mathrm{x}=0.0$ was relatively dependent on concentration. The SLP values of this sample increased by a factor of $\sim 1.5-2$ from higher concentrations to the concentration $c=0.75 \%$ and then for the lower concentrations no significant change was observed. The increase observed in the SLP of the sample $x=0.1$ was dramatically more pronounced. It increased by a factor of $\sim 5-6$ from higher concentration to the concentration $c=0.3 \%$ and then it remained unchanged for lower concentrations. Meanwhile, it should be noted that only the sample $\mathrm{x}=0.1$ showed a measurable temperature change at very low concentration $c=0.03 \%$ and for sample $\mathrm{x}=0.4$, for instance, no considerable temperature rise was observed at concentrations lower than $2.5 \%$ for frequencies of 137 and $240 \mathrm{kHz}$.

When comparing the SLP values among the samples of each concentration for all frequencies, the sample $\mathrm{x}=0.4$, which has the smallest size and the highest anisotropy constant value, showed the lowest SLP values. The sample $\mathrm{x}=0.1$ has the highest saturation magnetization, but size and anisotropy constant close to that of sample $\mathrm{x}=0$. This sample was the most efficient sample for almost all concentrations and frequencies. The sample $\mathrm{x}=0.2$ with a higher saturation magnetization and anisotropy, but smaller size than $\mathrm{x}=0$, at higher concentrations showed a higher SLP value than that of $\mathrm{x}=0$. However, by decreasing the concentration, SLP of sample $\mathrm{x}=0$ overtook that of sample $\mathrm{x}=0.2$. These results show a very complex dependence of SLP on different parameters such as size, anisotropy, saturation magnetization, and concentration. Meanwhile, there are other important parameters such as size distribution [289] or the effect of nanoparticle coating [290] which has not been considered in our study. 


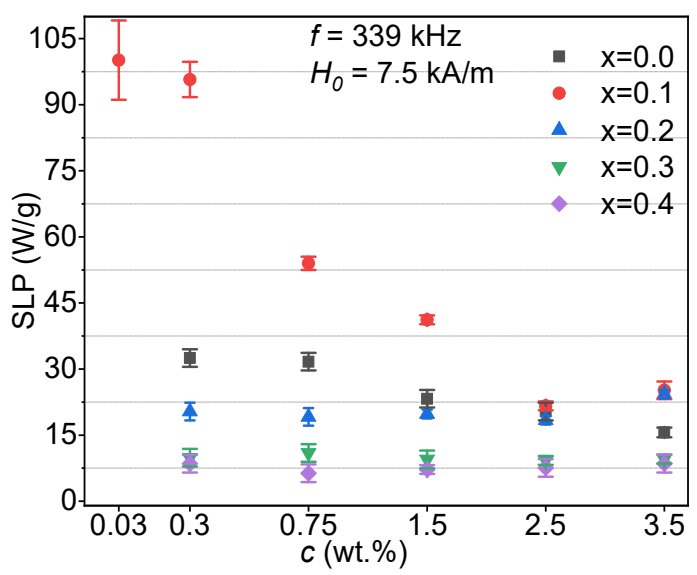

(a)

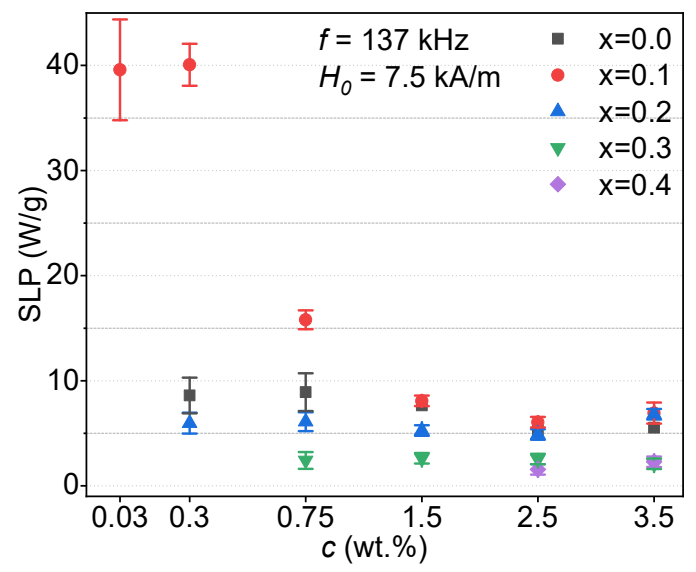

(c)

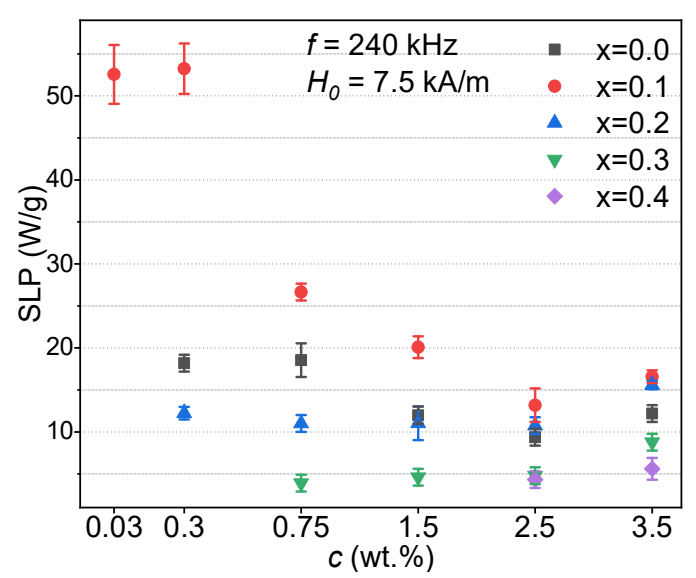

(b)

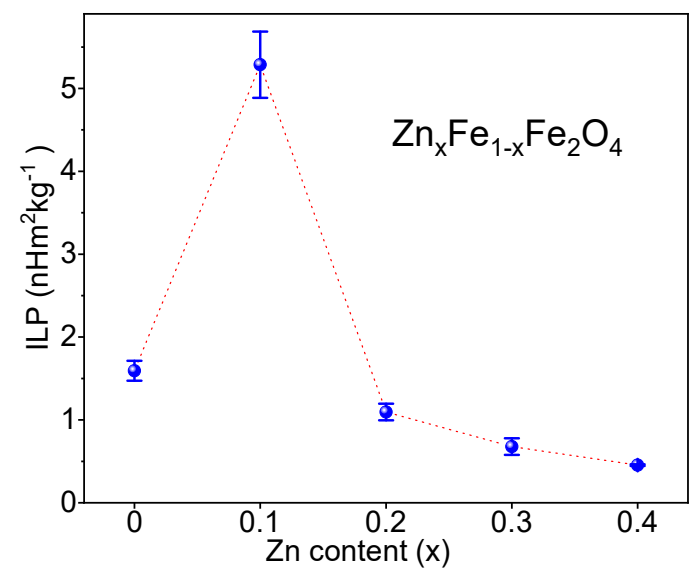

(d)

Figure 5.1 The measured SLP values of the samples at $H_{0}=7.5 \mathrm{kA} / \mathrm{m}$ and different concentrations for (a) 339 $\mathrm{kHz}$, (b) $240 \mathrm{kHz}$, and (c) $137 \mathrm{kHz}$. d) Mean ILP values considering all frequencies.

The intrinsic loss power (ILP) was introduced by Kallumadil et al. [229] to improve the comparison among the SLP values obtained using different magnetic field parameters. According to the linear response theory, ILP is calculated by normalizing the SLP values to $f$ and $H_{0}^{2}$. ILP values for all samples are shown in figure 5.1(d) with the highest value 5. $\mathrm{nHm}^{2} \mathrm{~kg}^{-1}$ for the sample $\mathrm{x}=0.1$. Besides the exceptional high ILP value reported for natural bacterial magnetosomes $\left(23.4 \mathrm{nHm}^{2} \mathrm{~kg}^{-1}\right)$ [291], for synthetic magnetic nanoparticles high values such as 8.7 [292], 7.4 [293], 6.1[294], and $4.1 \mathrm{nHm}^{2} \mathrm{~kg}^{-1}$ [252] have been reported, all well above commercially available magnetic nanoparticles [229]. Values above 3 are considered as suitable for magnetic hyperthermia [295]. 


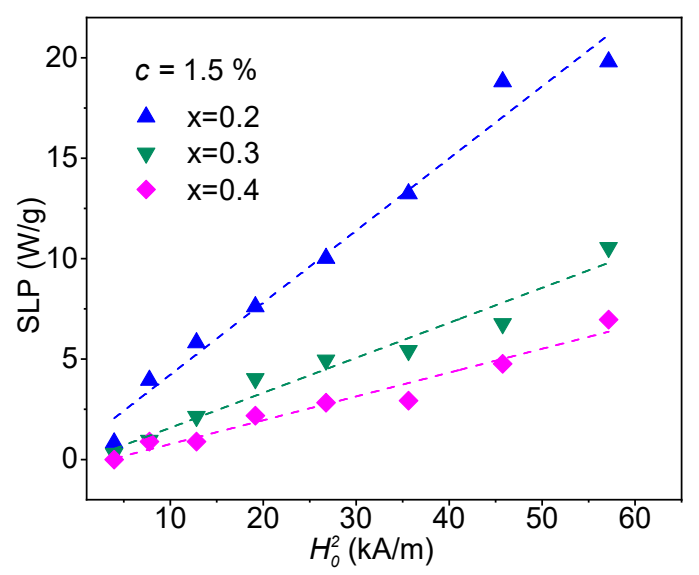

(a)

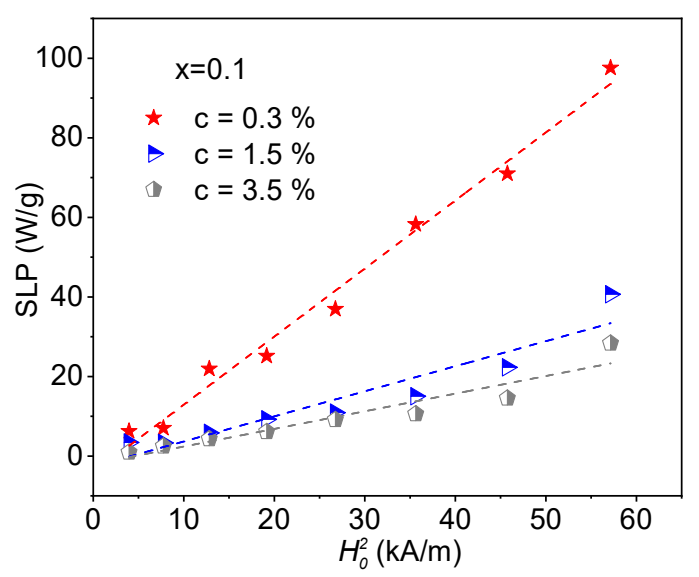

(c)

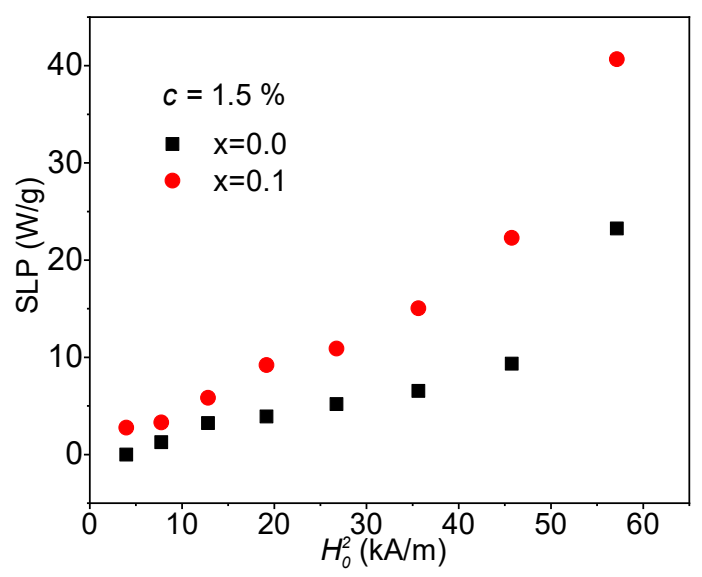

(b)

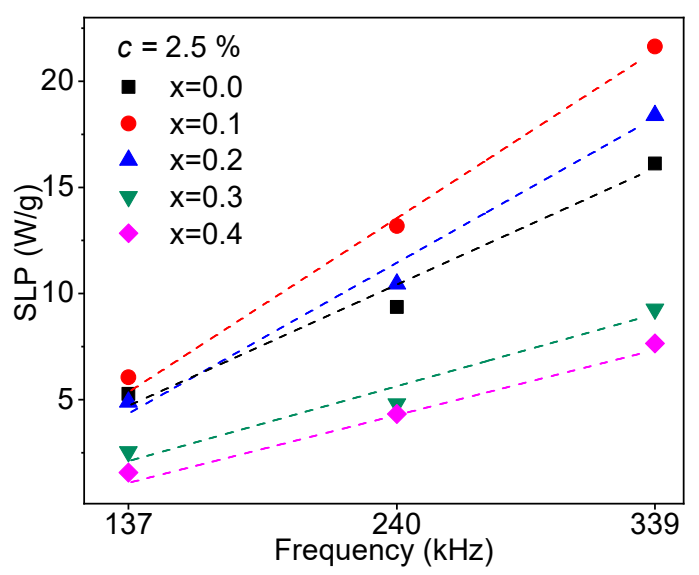

(d)

Figure 5.2 SLP field dependence (a) for sample $\mathrm{x}=0.2,0.3$ and 0.4 and (b) for sample $\mathrm{x}=0.0$ and 0.1 for $c=1.5$ wt.\%. (c) SLP field dependence for sample $\mathrm{x}=0.1$ and $c=3.5,1.5$, and $0.3 \mathrm{wt} . \%$. d) SLP frequency dependence for all samples at $c=2.5 \mathrm{wt} . \%$.

Since the criteria for linear response theory has been respected in all experiments of this study, SLPs are expected to be varied proportional to the square of the magnetic field amplitude. We chose the concentration $c=1.5 \%$ as a moderate concentration among our samples to study the field dependence of the SLP. The quadratic dependence was observed only for samples $\mathrm{x}=0.2$, 0.3 , and 0.4 (figure 5.2(a)), but not for samples $\mathrm{x}=0.0$ and 0.1 (figure 5.2(b)). For this reason, sample $\mathrm{x}=0.1$ was examined for two more concentrations. As it can be seen in figure 5.2(c), only for the lowest concentration sample the quadratic field dependence was observed. Such discrepancy has been reported in other studies [296, 297] and will be discussed in details in the succeeding sections. However, the SLP variations for all samples and concentrations, as 
predicted by the linear response theory, were directly proportional to the frequency. Figure 5.2(d) shows an example of this frequency dependency for $c=2.5 \mathrm{wt} . \%$.

\subsubsection{Numerical simulation results}

Figure 5.3 shows the dynamic hysteresis loops of the samples obtained using equation (5.5). Although all samples behaved as superparamagnetic in quasi-static fields [90], at higher frequencies they show an open elliptical shape loop which is the result of the lag between the magnetization with respect to the applied alternating field. This lag for samples $\mathrm{x}=0.3$ and 0.4 is such small that results in extremely low SLP values.

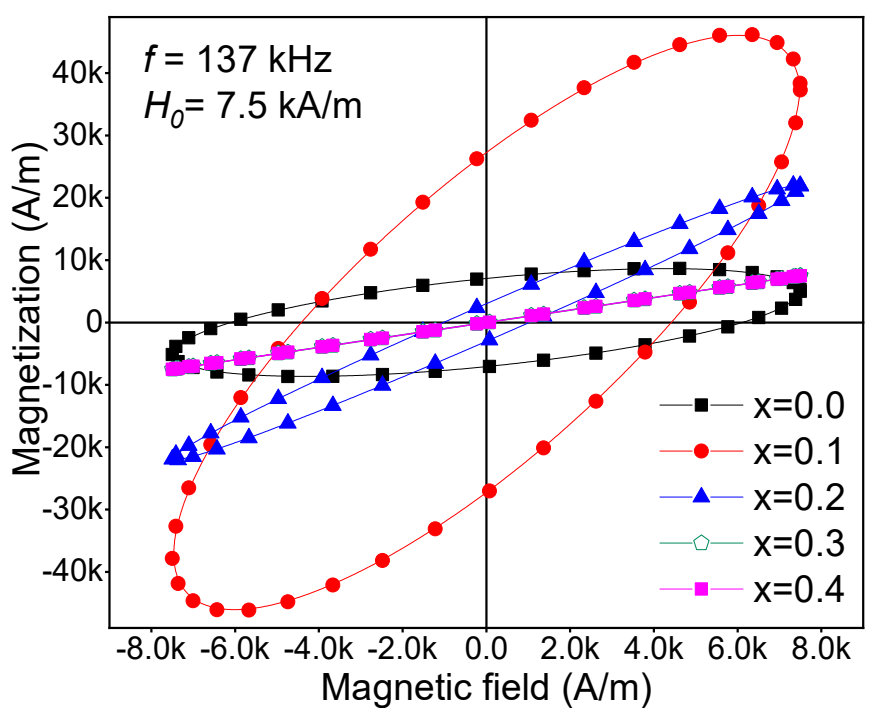

Figure 5.3 The dynamic hysteresis loops of the samples considering $f=137 \mathrm{kHz}$ and $H_{0}=7.5 \mathrm{kA} / \mathrm{m}$.

The dimensionless parameter $\sigma=E_{A} / k_{B} T=K_{\text {eff }} V / k_{B} T$ is an essential parameter directly influencing the relaxation time of the samples and hence their SLP values. Figure 5.4 shows the simulated variation of SLP versus $\sigma$ for each sample at different frequencies by considering the volume of the nanoparticles obtained from TEM and a wide range of $K_{\text {eff }}$ values. The dashed line in each figure represents the $\sigma$ value of the corresponding sample using the estimated $K_{\text {eff }}$ by the low of approach. Although the simulated SLP values were in good agreement with the experimental results at $\mathrm{c}=0.3 \%$ (see figure $5.4(\mathrm{f}$ ) as an example for $f=339 \mathrm{kHz}$ ), one should keep this in mind that these SLPs have been calculated considering the inverse attempt frequency of $10^{-9} \mathrm{~s}$ for all samples which is only a rough approximation of this parameter. 
Because $\tau_{0}$ strongly depends on intrinsic properties of the magnetic nanoparticles [251] and for each sample should be different.

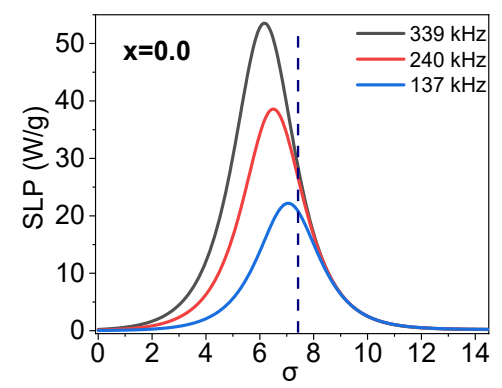

(a)

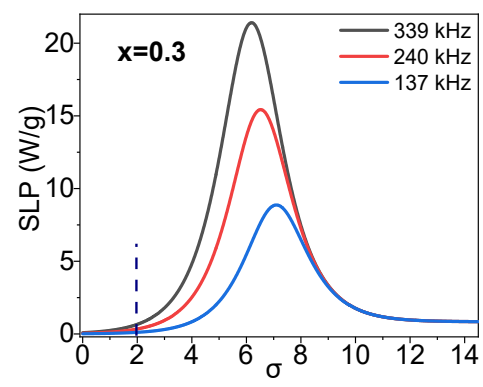

(d)

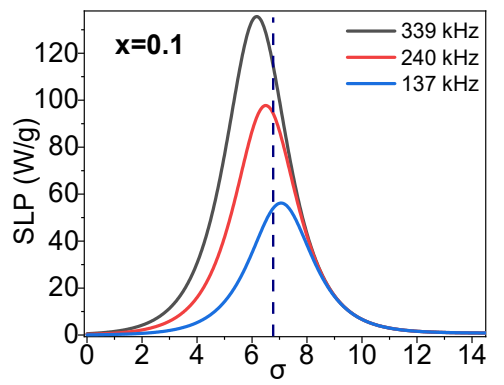

(b)

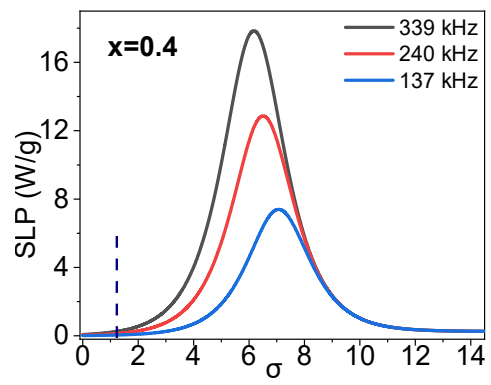

(e)

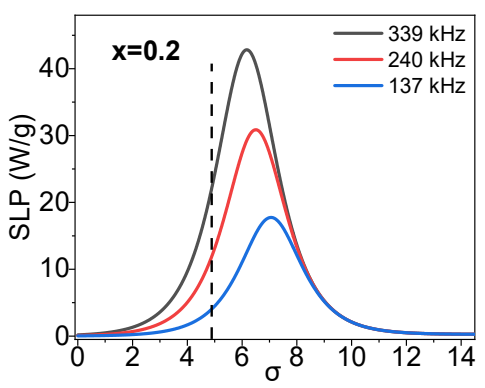

(c)

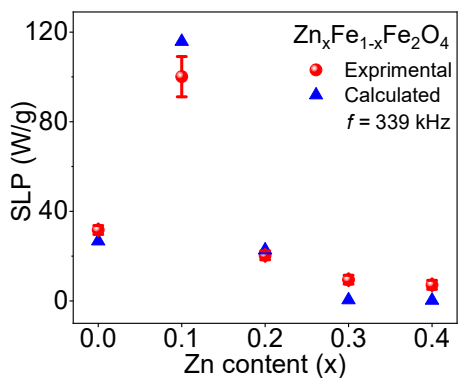

(f)

Figure 5.4 (a) - (e) SLP variation versus $\sigma=K_{\text {eff }} V / k_{B} T$ considering the volumes obtained from TEM images (the dashed lines represent the experimental values), and (f) the calculated and experimental values of SLP for all samples for $f=339 \mathrm{kHz}$ and at $c=0.3 \%$.

As it can be clearly seen in figure 5.4, there is a maximum SLP for each frequency which is shifted to lower values of $\sigma$ at higher frequencies. This is better shown in figure 5.5(a) where the SLP dependence on $\sigma$ and frequencies up to $1 \mathrm{MHz}$ is plotted for sample $\mathrm{x}=0.1$. SLP increases by increasing the frequency and its maximum occurs at different $\sigma$ for each frequency. However, at a fixed value of $\sigma$, SLP saturates at higher frequencies and it can be immediately understood from equation (5.2) for $2 \pi f \tau \gg>1$ [298]. This can be visualized in figure 5.5(b) as an example that shows the SLP dependence on frequency and field amplitude for the sample $\mathrm{x}=0.1$ with $\sigma=6.7$. 


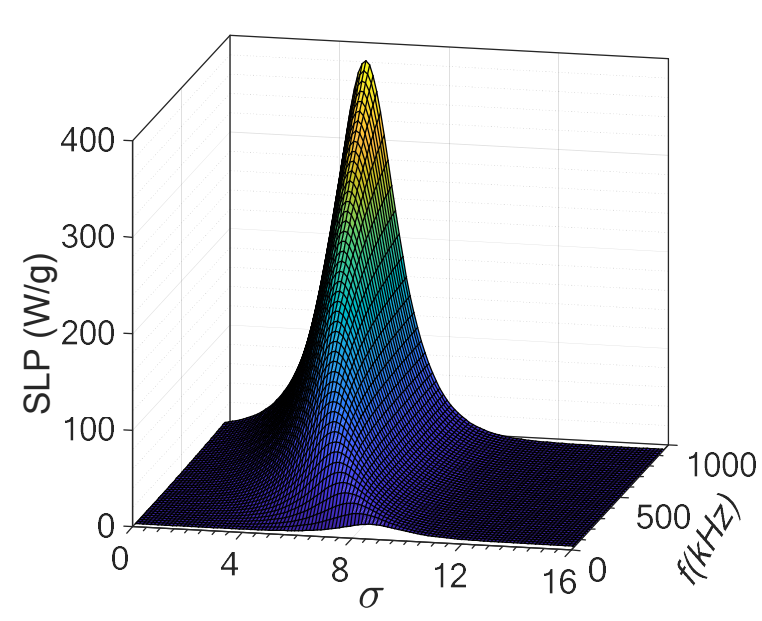

(a)

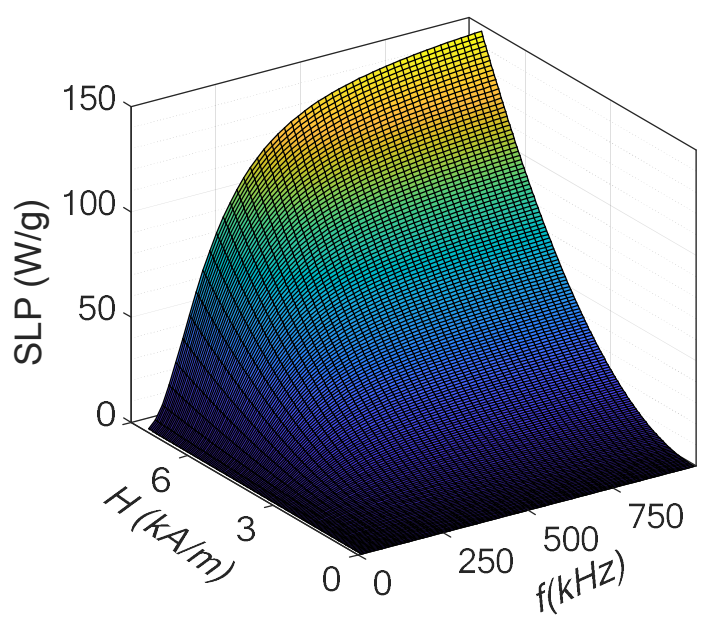

(b)

Figure 5.5 Simulated SLP versus (a) $\sigma$ and frequency for $H_{0}=7.5 \mathrm{kA} / \mathrm{m}$, and (b) field amplitude and frequency for $\sigma=6.7$.

\subsection{Discussion}

The linear response theory was chosen for numerical simulations since all experiments in this study were conducted at relatively low field amplitudes, which respect the condition $\mu_{0} H_{0} M_{s} V \leq k_{B} T$ for all samples. Moreover, as it was pointed out by Ruta et al. [240], the ratio between $H_{0}$ and the anisotropy filed $\left(H_{K}=2 K / \mu_{0} M_{s}\right)$ of the sample can also determine the applicability of the linear response theory. For values $H_{0} / H_{K}<<1$, the linear response theory can quantify the experimental results. In the present study, for all samples this ratio was in the order of $10^{-2}$.

\subsubsection{Interparticle interactions}

In the framework of the linear response theory, in addition to assuming a monodispersed magnetic nanoparticles system, no contribution from dipolar interactions between the particles has been taken into account. However, in real conditions these two assumptions are rarely met and there may always exist a degree of polydispersity [299] and also interparticle interactions. Here, we assume the first assumption is true and try to interpret the observed results with the hypothesis that the variations in the SLP values with concentration are related to interparticle interactions. This hypothesis is relevant, especially because interparticle interaction strongly depends on interparticle distances and this varies with the concentration in a colloidal dispersion. 
As mentioned above, apparently very different theoretical and experimental results regarding the effect of interparticle interactions have been reported and this discussion still appears to be not conclusive. For example, in two very recent studies interparticle interactions have shown to significantly increase [300] and decrease [301] the heating efficiency of the system. In our results, however, two distinguishable trends can be recognized. For sample $\mathrm{x}=0.0$ and 0.1 , decreasing the concentration, which means increasing the interparticle distances and hence reducing the strength of the interactions, has led to an increase in their SLP values. On the other hand, for the other three samples their heating efficiencies have not been influenced by this effect. Presa et al. [257], using maghemite nanoparticles with different sizes, showed that even from extremely high concentrated sample $(\sim 30 \%)$ to the low concentration of $0.6 \%$, the SLP values remained unchanged. They supposed that either the dipolar interactions are not significant to influence the heating efficiency or due to the existence of clusters, dilutions will not change the effect of the interactions. We believe in our results the former explains the monotonous behavior observed in samples $\mathrm{x}=0.2,0.3$, and 0.4 . This will be discussed in more details in the following paragraphs.

Although most of the theoretical studies show a negative effect of interparticle interaction on hyperthermia performance of magnetic nanoparticles [251, 254, 255, 302], several studies have shown that depending on intrinsic properties of the nanoparticles, interactions may increase or decrease the heating efficiency of the system [246, 296, 303]. Tan et al. [246], for example, using Monte Carlo simulations showed that the heating efficiency of the nanoparticles, depending on the relation of the applied magnetic field with the coercivity or saturation field of the nanoparticles, may increase, decrease, or behave in a way they called as bell shape. They also showed the effect of interactions is more pronounced for magnetic nanoparticles with lower anisotropy. In references [247, 256, 259], the authors have shown that for certain sizes and shapes of the formed clusters or chains in colloidal dispersions, SLP may increase or decrease. They showed that for clusters that are anisotropic in shape, there will be an extra anisotropy induced to the system leading to an increase in the SLP values. While an isotropic configuration has a negative influence on the heating efficiency compared to non-coupled nanoparticles. For samples $\mathrm{x}=0.0$ and $\mathrm{x}=0.1$ of the present study, we also believe the pronounced effect of interparticle interactions arising from the large magnetic moments of their single domain particles can explain the observed behavior. 
In a colloidal dispersion of single domain magnetic nanoparticles with the magnetic moment $\mu=V M_{s}$, the maximum magnetic interaction potential between two neighboring particles in zero applied magnetic field is given by [299]:

$$
U_{\max }=\frac{\mu_{0} \mu_{i} \mu_{j}}{2 \pi d_{i j}^{3}}
$$

where $d_{i j}=\left(d_{i}+d_{j}\right) / 2$ with $d_{i}$ and $d_{j}$ the diameters and $\mu_{i}$ and $\mu_{j}$ are the magnetic moments of the particles $i$ and $j$. To characterize the interparticle interactions, the parameter $\lambda$ has been introduced as dipolar coupling constant which relates $U_{\max }$ for two particles in contact with one another to the thermal energy as:

$$
\lambda=\frac{\mu_{0} \mu^{2}}{2 \pi d^{3} k_{B} T},
$$

where $\mu$ and $d$ are the mean magnetic moment and mean diameter, respectively. For $\lambda>2$ the effect of the interactions is non-trivial, the system is considered as strong interacting, and aggregates may happen. When $\lambda<2$ the system is said to be in homogenous "gaslike" state and the interparticle interactions are negligible [299]. The calculated $\lambda$ values for our samples (figure 5.6(a)) show that only samples $\mathrm{x}=0.0$ and 0.1 are within the strong interacting limit.

Moreover, a qualitative analysis of the interparticle interaction energy at different concentrations can also demonstrate how pronounced the effect of interaction for the samples $\mathrm{x}=0.0$ and $\mathrm{x}=0.1$ is compared to the other three samples. The maximum magnitude of the dipolar interaction energy of two neighboring particles with the mean interparticle distance $r$ from one another can be expressed as [304]:

$$
E_{\mathrm{int}}=\frac{\mu_{0} \mu^{2}}{4 \pi r^{3} k_{B} T} .
$$

By assuming perfect and rigid spherical particles, the interparticle distance of a colloidal dispersion sample with volume $V_{s}$ and consisting of $N$ particles can be obtained as $r=\left(V_{s} / N\right)^{1 / 3}$. Although equation (5.11) considers only two neighboring particles, it can qualitatively be an indication of the magnitude of the interparticle interactions in the whole system. Quantitative comparison implies more considerations and extensive computational methods (see for example [254]) which is beyond the scope of the present study. Considering all concentrations and using the mean particle diameters of our samples in the volume of $300 \mu \mathrm{L}, E_{\text {int }}$ was 
calculated. Figure 5.6(b) shows this quantity (relative strength of the dipolar interaction) for all samples at all concentrations. As it can be seen, interactions in samples $\mathrm{x}=0.0$ and 0.1 are higher than the other three samples at higher concentrations and at the lowest concentration all samples seem to act as non-interacting particles.

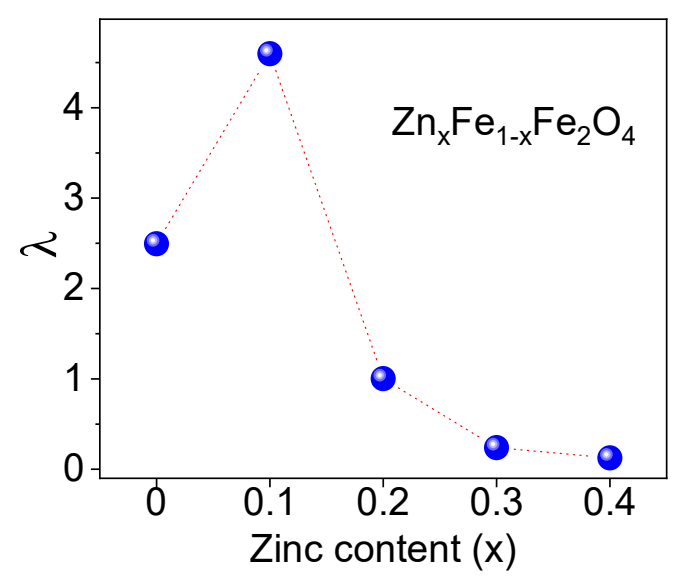

(a)

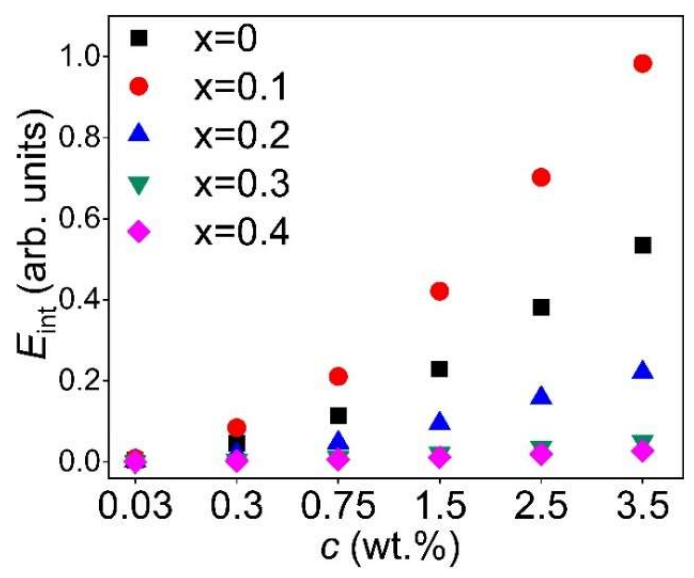

(b)

Figure 5.6 Dipolar coupling constant $(\lambda)$ and (b) relative strength of the dipolar interaction at different concentrations.

According to the preceding discussions, we can conclude that two different trends of heating efficiencies versus concentration can be related to the different effect of interparticle interaction in the samples. As stated earlier, it has been proven that chain and cluster formation in interacting particles will introduce an additional anisotropy to the system [251, 256, 259, 260]. Moreover, theoretical and experimental evidences have shown that interparticle interaction considerably increases the energy barrier in single domain magnetic nanoparticles [272, 305307]. Having this in mind and recalling the results shown in figure 5.4, the reason for the observed trends in SLP values can be qualitatively understood by considering: i) the calculated SLP values versus the parameter $\sigma$ has been obtained using the linear response theory without considering the effect of interparticle interactions and ii) the increase in the energy barrier directly leads to a shift in parameter $\sigma$ to higher values. This effect can be qualitatively evaluated for sample $\mathrm{x}=0.0$ and 0.1 in figure 5.4 by shifting the dashed lines to the right side for higher concentrations. In fact, here in samples $\mathrm{x}=0.0$ and 0.1 increasing the parameter $\sigma$, increases their relaxation times and hence leading to more deviation from the maximum heating efficiency condition of $2 \pi f \tau=\omega \tau=1$ at each given frequency [308]. For these two samples, the 
product $\omega \tau$ is higher than 1 for all frequencies except for $f=137 \mathrm{kHz}$ sample $\mathrm{x}=0.1$; though close to 1 . These results are in complete agreement with previous theoretical study by Landi based on a mean-field model [245].

Although for the other samples increasing the parameter $\sigma$, apparently, should have a positive effect on their SLP, such an effect was not clearly observed. This is most likely due to the negligible strength of the interparticle interactions in these samples. Tan et al. [246], in their theoretical study on the effect of interparticle interactions, related the SLP values to the dimensionless concentration parameter expressed as $\mu_{0} M_{s}^{2} c / K_{\text {eff }}$. In their results, for values higher than 0.02, interparticle interactions became noticeable. In our experimental results, SLP for sample $\mathrm{x}=0.0$ at concentrations below $c=1.5 \%$ and sample $\mathrm{x}=0.1$ at concentrations below $c=0.75 \%$ was almost not affected by concentration. The corresponding calculated dimensionless concentration for these two conditions is 0.06 which can be considered as the threshold value for our samples. Dimensionless concentration for all other samples, at all concentrations, were well below 0.06 except for sample $\mathrm{x}=0.2$ at $c=3.5 \%$ which is around 0.06 .

Another feature of the experimental results, which may be explained by considering the interparticle interactions, is the discrepancy from quadratic field dependence of SLP values at higher concentrations for samples $\mathrm{x}=0.0$ and 0.1 . Carrião et al. [241] showed that depending on the changes in the parameter $\sigma$ and hence the relaxation time from its optimum value $(\omega \tau=1)$, different deviation from the quadratic field dependence may happen. Here, a qualitative analysis can also be conducted using the observed field dependence of SLP for sample $\mathrm{x}=0.1$ at two concentrations $c=3.5 \%$ and $1.5 \%$ in order to show the changes in $\sigma$. By simulating the $\operatorname{SLP}\left(H_{0}\right)$ variation versus $\sigma$ using the same field amplitudes as in the experiments and then correlating these simulations with the observed experimental SLP $\left(H_{0}\right)$ values, new values for parameter $\sigma$ can be estimated. The estimated mean values were $8.4 \pm 0.2$ and $8.9 \pm 0.2$ for concentrations $c=1.5 \%$ and $3.5 \%$, respectively, which shows a considerable increase due to the interparticle interactions compared to that obtained using $K_{\text {eff }}$ of this sample $(\sigma=6.7)$. However, it is worth noting that from these values no clear conclusion about the induced anisotropy to the system can be drawn, because at the presence of the applied magnetic field, the height of the energy barrier is the result of the relative interplay between the anisotropy, Zeeman, and interaction energy in a complex manner [306]. Therefore, when employing the linear response theory to interpret the experimental results, in addition to respecting the defined limit for linear 
regime i.e. $\mu_{0} H_{0} M_{s} V \leq k_{B} T$, a good knowledge of interparticle interactions seems to be crucial too.

\subsubsection{Heating efficiency}

Overall, the samples under study here present a range of different intrinsic properties. The results show that their heating efficiency does not depend on a single intrinsic property, but on a variety of parameters. Interpreting the results considering only one parameter, for example, size or anisotropy may be misleading. These two characteristics, especially at nanoscale, do not vary independently of one another. Instead, the parameter $\sigma$, which is a deterministic quantity for determining the relaxation time of the particles, is more appropriate to be discussed. In a fixed field frequency and amplitude, for a magnetic nanoparticles system with a known saturation magnetization, and in linear regime this parameter determines the heating efficiency of the system; i.e. how close the system is to the condition $\omega \tau=1$. In our samples, for example, although there is no considerable difference in the saturation magnetization of samples $\mathrm{x}=0.0$ and $\mathrm{x}=0.3$ and 0.4 , their SLP values are considerably different. This can be seen from the position of their $\sigma$ in the graphs shown in figure 5.4. On the other hand, samples $\mathrm{x}=0.0$ and $\mathrm{x}=0.1$ present similar $\sigma$ values, and the dramatic difference in their heating efficiency arises from the difference between their saturation magnetization values. The importance of the parameter $\sigma$ can be better understood by comparing the SAR values of sample $\mathrm{x}=0.0$ and 0.2 , where the latter has a higher saturation magnetization but a smaller $\sigma$. The sample $\mathrm{x}=0.0$, in most of the concentrations studied, showed a better performance than $\mathrm{x}=0.2$.

As a final remark, we recall the results shown in figure 5.5 (a) as a typical example of the dependence of SLP on the field frequency and $\sigma$. By increasing the field frequency, the peak positions in this figure are shifting to the lower $\sigma$ values. Considering the parameter $\sigma$ as a fixed intrinsic constant, the condition $\omega \tau=1$ occurs only at a certain frequency for each kind of magnetic nanoparticles. Therefore, any frequency higher or lower than this frequency leads to a less efficient energy conversion. Figure 5.7 shows this energy conversion efficiency for all samples analyzed in this study. As it can clearly be seen, only for samples $\mathrm{x}=0.0$ (at $100 \mathrm{kHz}$ ) and 0.1 (at $150 \mathrm{kHz}$ ) the maximum energy conversion efficiency can occur at usable frequencies for clinical trials. Moreover, the larger effective anisotropies in samples $\mathrm{x}=0.2,0.3$, and 0.4 imply higher field amplitudes to achieve a reasonable SLP value and this is also another limitation for these kind of samples to be used in clinical magnetic hyperthermia. In such situations, due to safety considerations, increasing the field frequency or amplitude is not 
possible; therefore, tuning the intrinsic magnetic properties of the nanoparticles is the preferred strategy.

Cation substitution in ferrite nanoparticles has always been considered as a very straightforward strategy to optimize their structural and magnetic properties. In the present study, zinc-substituted magnetite ( sample $\mathrm{x}=0.1$ ) showed superior SLP values compared to the magnetite nanoparticles with a relatively high ILP value. In our previous study [90], this composition improved the signal to noise ratio in magnetomotive ultrasound imaging technique as a diagnostic application, which shows the great potential of the sample $\mathrm{x}=0.1$ to be considered as a theranostic agent in biomedical applications. Indeed, theranostic is the fusion of diagnostic and therapeutic procedures in a single nanoparticles system where the same magnetic nanoparticles can serve, simultaneously, as an image contrast agent and to deliver targeted chemotherapeutic agents directly to the cancer cells or as heating mediator in magnetic hyperthermia [309, 310].

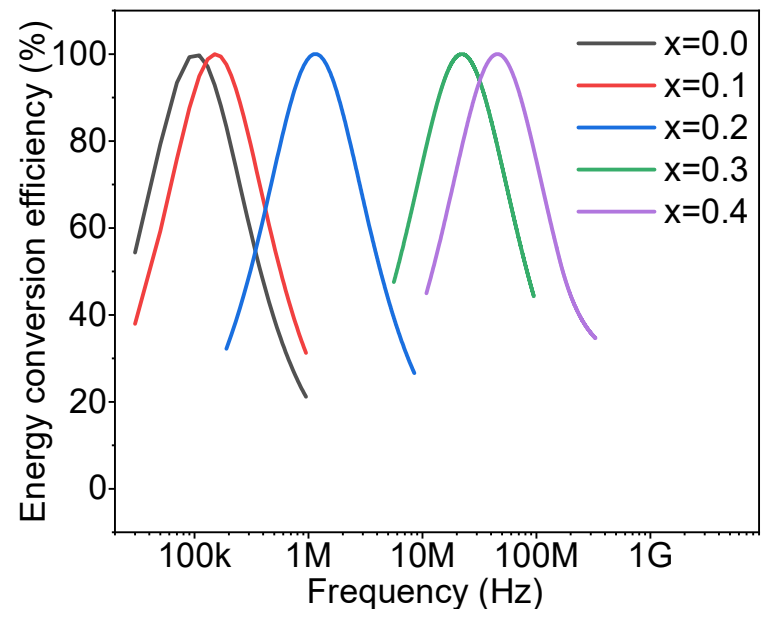

Figure 5.7 Energy conversion efficiency versus frequency for all samples.

\subsection{Summary}

In summary, zinc-substituted magnetite nanoparticles were examined in various experimental conditions for magnetic hyperthermia. Due to different intrinsic properties of these compositions, they showed to be a very useful tool to observe the effect of parameters such as saturation magnetization, anisotropy and size on the heating efficiency of the magnetic nanoparticles. The sample $\mathrm{Zn}_{0.1} \mathrm{Fe}_{0.9} \mathrm{Fe}_{2} \mathrm{O}_{4}$ showed a relatively high ILP value $\left(5.4 \mathrm{nHm}^{2} \mathrm{~kg}^{-1}\right)$ 
compared to the other reports on $\mathrm{Zn}_{\mathrm{x}} \mathrm{Fe}_{1-\mathrm{x}} \mathrm{Fe}_{2} \mathrm{O}_{4}$ composition in the literature $(0.5$ [121], 1.57 [311], and 2.36 [265] $\mathrm{nHm}^{2} \mathrm{~kg}^{-1}$ ), which offers a great potential to be considered as a theranostic agent. Although Srivastava et al., recently, reported ILP values ranging from 1.16 to 13.76 $\mathrm{nHm}^{2} \mathrm{~kg}^{-1}$, comparing their results with ours is quite tricky. Because ILP parameter is only valid in the framework of linear regime, while in that study high fields up to $20 \mathrm{kA} / \mathrm{m}$ were used. Furthermore, the different intrinsic properties had diverse effects related to the dipolar interactions on the heating efficiency of the particles. For linear regime, this study resulted in two main conclusions: i) at the same sample concentration the strength of dipolar interactions is determined by the intrinsic properties; ii) if the dipolar interactions are strong enough to affect the heating efficiency, the parameter $\sigma$ determines the type of the effect. As interactions increase the parameter $\sigma$, for any $\sigma$ value resulting in $\omega \tau<1$, interactions can increase the heating efficiency. On the other hand, for $\omega \tau \geq 1$ interactions have the opposite effect. This shows also the importance of choosing the appropriate frequency to increase the heating efficiency of high interacting magnetic nanoparticle system. We believe these results, considering the wide range of intrinsic properties and experimental conditions investigated, may have important implications for optimizing the heating efficiency of magnetic nanoparticles in hyperthermia. 


\section{Chapter 6 : A novel theranostic platform: integration of magnetomotive and thermal ultrasound imaging with magnetic hyperthermia}

\subsection{Introduction}

Theranostic systems, the fusion of diagnostic and therapeutic procedure in a single platform, have shown a great potential to improve the efficiency of cancer treatment. For example, a single magnetic nanoparticle composition can serve, simultaneously, as contrast agent in magnetic resonance imaging (MRI) to detect the tumor and to deliver targeted chemotherapeutic agents [309], or as heating mediator for magnetic hyperthermia [312]. Although much effort has, recently, been devoted to develop nanotheranostic agents, little attention has been paid to the development of instrumentation with theranostic purposes.

The use of conversion of the electromagnetic energy to heat, through fine magnetic particles, for treating cancer was first introduced by Gilchrist et al. in 1957 [55]. Since then, this method, known as magnetic hyperthermia, has been extensively investigated and shown a great potential to selectively induce apoptosis and/or necrosis in cancerous cells [99]. In fact, in magnetic hyperthermia, thermal energy is released during the magnetization reversal process in magnetic nanoparticles when exposed to a radiofrequency magnetic field. Since cancer cells are more vulnerable to heat than normal cells, due mostly to incomplete development of vascular and nervous systems in tumors, sustained abnormal temperatures $\left(43-46^{\circ} \mathrm{C}\right)$ can kill them $[313,314]$. On the other hand, organized and systematic blood flow in healthy tissues can effectively inhibit excessive heat allowing normal cells to survive in environments with temperatures up to $46{ }^{\circ} \mathrm{C}$; this defines a temperature window for magnetic hyperthermia therapy [313].

Research on magnetic hyperthermia including optimizing the intrinsic properties, functionalizing, and targeted delivery of the magnetic nanoparticles for in vitro and in vivo studies are growing exponentially [57]. Despite substantial progresses, there still exist several challenges that hinder this technique to be effectively translated to a clinical routine. For example, development of instruments capable of delivering strong radiofrequency in large 
volumes is not trivial and can be costly. Localizing magnetic nanoparticles within the tissue and monitoring the temperature during the treatment procedure are other major concerns. The two latter are of crucial importance, because either accumulation of the magnetic nanoparticles in healthy tissues or excessive heating may result in non-desired tissue damage.

It has been proven that MRI [315] and magnetic particle imaging (MPI) [316, 317] can efficiently localize the magnetic nanoparticles; however, these techniques are not easily integrated with magnetic hyperthermia and are high-cost techniques. Hensley et al. [77] for the first time combined a MPI system with a magnetic hyperthermia applicator to localize magnetic nanoparticles and selectively heat the region of interest (ROI). The authors evaluated the theranostic platform using a triple-vial phantom. In addition to localizing the magnetic nanoparticles, MRI has successfully been used to monitor the temperature during different thermal therapies [318-323]. Although MR thermometry is real-time and provides the temperature map in a wide range of temperatures, it is a very complex and high-cost technique [324]. Recently, Huang et al. [325, 326] used magnetomotive optical coherence elastography to monitor the thermal dose delivered by magnetic hyperthermia, based on alteration of the viscoelastic properties of tissue, during the therapy. However, the temperature was measured only on the surface of the samples and using either a digital thermometer or a thermal camera. In addition, optical coherence tomography is a superficial imaging technique.

Magnetomotive ultrasound (MMUS) imaging is another technique which has been used to detect magnetic nanoparticles within tissues [44]. This technique uses ultrasound echo signals to localize and detect microvibrations of the medium labeled with magnetic nanoparticles, which are the result of the interaction between magnetic nanoparticles and the applied magnetic field. The induced displacement in MMUS depends directly on the amplitude and the gradient of the applied magnetic field and the magnetization and concentration of magnetic nanoparticles. In vivo [48] and phantom [90] studies have shown that using magnetic nanoparticles with enhanced saturation magnetization $\left(\mathrm{M}_{\mathrm{s}}\right)$ can considerably increase the signal to noise ratio (SNR) in MMUS imaging. In addition, this method uses non-ionizing radiation, is cost effective, can be used to take images at relatively high depths, and can be easily combined with other techniques [41, 170, 174, 175, 327, 328]; therefore, it has attracted increasing attention.

Thermal strain imaging is another ultrasound based technique that offers noninvasive temperature monitoring in biomedical studies. It was originally proposed to monitor the 
temperature and to differentiate lipid pools and lipid-bearing tissues from water-bearing tissues [329]. As the temperature is increased, it induces various changes in the intrinsic acoustic properties of tissues. For example, temperature induced variations in the speed of sound result in time shifted backscattered echo signals [330]. Monitoring these small variations in acoustic wave time-of-flight, using the data collected with conventional ultrasound scanners, can be used to estimate the temperature variation and hence non-invasively providing a temperature map within tissue. Thermal strain imaging has been used to monitor the temperature in different applications such as high intensity focused ultrasound hyperthermia [324,331], radiofrequency ablation [332], microwave absorption [333], and photothermal cancer therapy [334].

Given the aforementioned challenges in magnetic hyperthermia, the present study aimed to examine the possibility of addressing two main issues (i.e. localizing the magnetic nanoparticles and measuring the temperature during hyperthermia) in an integrated theranostic platform. This system was designed for preclinical magnetic hyperthermia therapy investigations using phantom or small animals. It is composed of an ultrasound imaging device, a magnetic hyperthermia system, a current pulse generator, and a pancake coil. The ultrasound device can be used to acquire morphological images, MMUS to detect the magnetic nanoparticles, and ultrasound thermometry to monitor the temperature within tissue during magnetic hyperthermia. The same pancake coil was used in both applications, i.e. to generate the field for magnetic hyperthermia and for MMUS. However, the resultant field was a radiofrequency field $(106 \mathrm{kHz})$ for magnetic hyperthermia, while it was a millisecond magnetic field pulse to induce the necessary motion to generate the MMUS images. The proposed system was tested with a gelatin/agar tissue-mimicking phantom containing an inclusion filled with magnetic nanoparticles. In addition, to the best of our knowledge, this is the first paper reporting ultrasound thermometry to monitor the temperature during magnetic hyperthermia. The unique feature of this platform is that the diagnostic and therapeutic applications are achieved through a single coil and a single magnetic nanoparticle composition, which enables the entire system to be used as a theranostic platform.

\subsection{Materials and methods}

The two main components of the developed theranostic platform are the magnetic excitation systems and the ultrasound-based imaging techniques. Magnetic excitation was used in i) therapeutic hyperthermia, where radiofrequency magnetic field was employed to induce heat dissipation through the interaction with magnetic nanoparticles; and in ii) MMUS imaging, where a millisecond pulsed magnetic field was responsible for inducing motion to magnetic 
nanoparticles, consequently displacing the surrounding tissues. The electronics for the magnetic excitations were developed in our laboratory, so that the same coil could be used for both magnetic hyperthermia and MMUS imaging.

Three different ultrasound-based imaging techniques were explored using the same ultrasound scanner and transducer. i) Conventional B-mode ultrasound images to assess structural information; ii) MMUS images to locate the magnetic nanoparticles by detecting magnetomotive induced motion using ultrasonic echoes; iii) ultrasound thermal strain imaging, generated by detecting the variations in the speed of sound, to monitor the temperature during the hyperthermia procedure.

Figure 6.1 shows a schematic of the theranostic platform developed in this study. As it can be seen, the same coil was driven by two different sources. For magnetic hyperthermia, the power supply was a resonant tank circuit working with continuous wave $(\mathrm{CW})$ radiofrequency current; while, for MMUS the coil was connected to a capacitor bank. In this case, the capacitors were discharged through the coil to produce a magnetic field pulse with time duration of a few milliseconds. Ultrasound data were acquired during magnetic excitation in both cases. The details of each part depicted in figure 6.1 will be explained in the following paragraphs. 


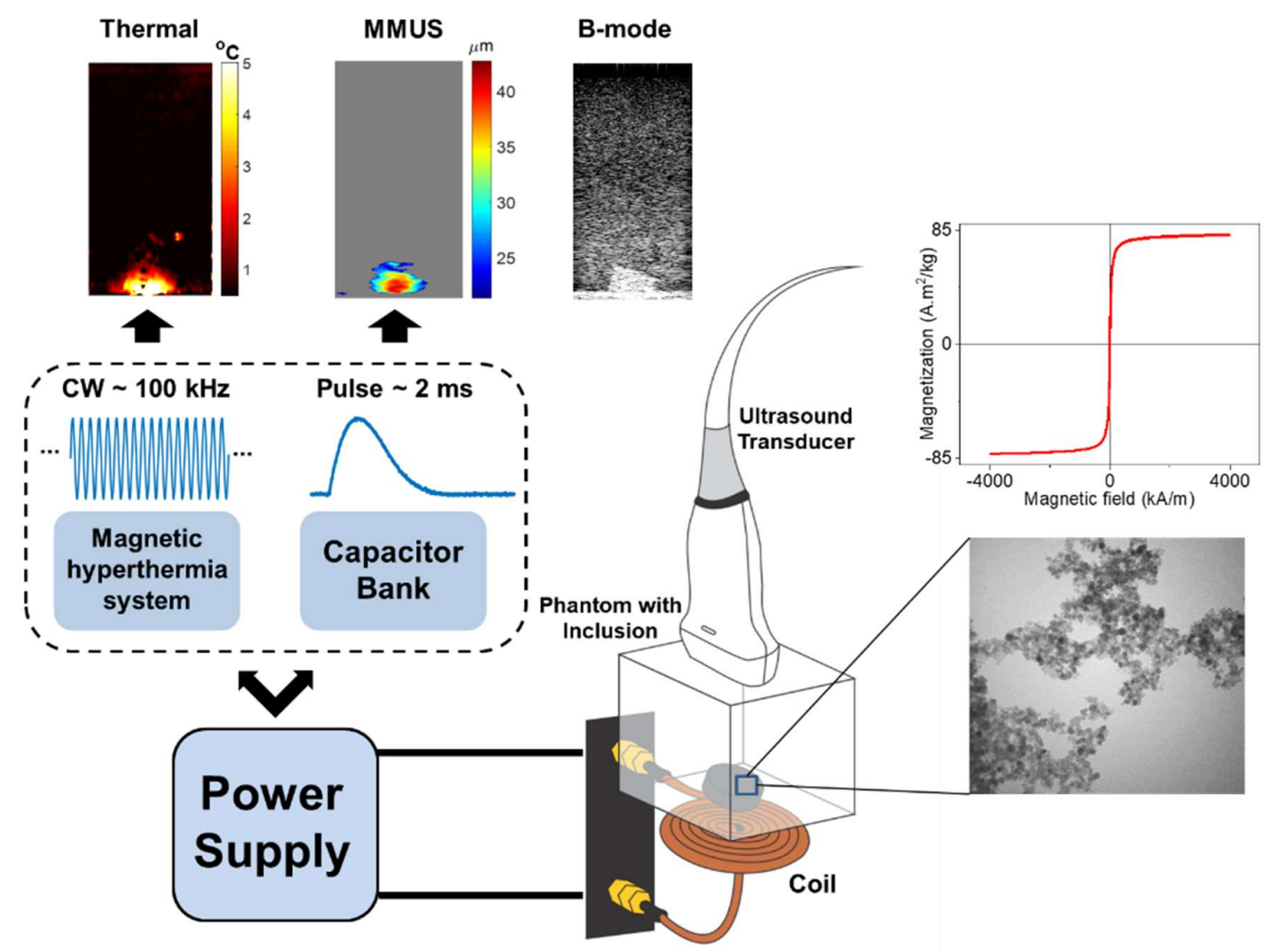

Figure 6.1 A schematic depiction of the proposed theranostic platform. The pancake coil was driven by two different power supplies to generate a pulse (for MMUS) and a radiofrequency magnetic field (for magnetic hyperthermia). In both procedures, a clinical ultrasound device was employed to acquire the MMUS and thermal images.

\subsubsection{Pancake coil}

Solenoid coils are widely used for magnetic hyperthermia experiments; however, they may lack adequate space for small animal experiments. Increasing the diameter of a solenoid coil carrying a fixed current significantly decreases the field amplitude and homogeneity [335]. In addition, for conditions that a strong magnetic field focused in a small region is needed, solenoid is not a proper format. Instead, pancake coils can be used to generate strong magnetic fields focused in a sphere volume, co-centered with the coil, which allows selective application of the magnetic field $[92,202,237]$. Therefore, this format can be considered as an interesting coil geometry in certain conditions $[202,237,336]$. The coil used in this study was a pancake coil manufactured by 3/16" ( $\sim 76 \mathrm{~mm})$ diameter copper refrigerator tubing isolated with heatshrink tube. It was composed of 7 turns, resulting in $10 \mathrm{~mm}$ and $80 \mathrm{~mm}$ inner and outer 
diameter, respectively, and $1.7 \mu \mathrm{H}$ inductance. The 3D magnetic field profile for this coil has been reported elsewhere [92].

\subsubsection{Magnetomotive ultrasound imaging}

As mentioned before, magnetic nanoparticles immersed in a phantom or tissue can be attracted by an external magnetic field; therefore, moving the magnetic nanoparticle-laden regions. The magnitude of this motion depends on the magnetic field amplitude and its gradient, magnetic nanoparticles magnetization and concentration, and the viscoelastic properties of the medium [44]. The magnetic force due to the interaction of an applied magnetic field $\left(H=B / \mu_{0}\right)$ with magnetic nanoparticle having the magnetization $M=\chi H$ and the volume $V$ in one dimension $(z)$ can be approximated as [176]:

$$
F(z)=\chi V H \frac{d B_{z}}{d z}
$$

where $\chi$ is the susceptibility of magnetic nanoparticle.

To generate the pulsed magnetic field for MMUS, the pancake coil was energized by discharging the capacitor bank with $48 \mathrm{mF}$ capacitance and a maximum voltage of $320 \mathrm{~V}(2.5$ $\mathrm{kJ}$ storage energy). Basically, the capacitors were charged using a power supply and discharged through a silicon controlled rectifier (SRC), which is turned on when a current pulse is applied to its gate. In the present study, the capacitor bank was charged using voltages below $50 \mathrm{~V}$ and the magnetic field created during the MMUS experiments was measured using a gaussmeter (TMAG-1T, Globalmag, Cotia, SP, Brazil). The external pulsed voltage used to trigger the gate driver was also responsible to trigger the ultrasound system. This is a strategy very similar to that employed in magnetic nerve stimulation, e.g. transcranial magnetic stimulation, where an intense magnetic pulse is applied to noninvasively excite the nerves [337, 338].

An ultrasound machine (RP Sonix, Ultrasonix Medical Corporation) equipped with a 128 elements linear array transducer (L14-5/38) was used to acquire the ultrasonic radiofrequency (RF) data to generate the B-mode, MMUS and thermal strain images. To acquire the MMUS images, the ultrasound transducer emitted unfocused plane waves and the backscattered echoes were acquired as pre-beamformed RF data using a multi-channel data acquisition system (DAQ, Ultrasonix Medical Corporation). This strategy allowed the acquisition of 4000 frames per second, which is much higher than the frame rate achievable using the conventional beamforming adopted by the clinical software. This is a crucial prerequisite since the induced 
motion by the short-duration ( $\sim 2 \mathrm{~ms})$ magnetic excitation would not be correctly sampled at lower frame rates. This RF data were reconstructed using a delay and sum algorithm.

A speckle tracking strategy was used to estimate the induced displacements within the phantom, hence, generating the MMUS images. In this method, the time shift between the RF data of a reference frame and the subsequent frames, collected during the applied pulsed magnetic field, was estimated using an axial 1-D normalized cross-correlation algorithm [180, 339]. This algorithm was applied to $0.8 \mathrm{~mm}$ windows with $70 \%$ overlap. The time delay between two windows was estimated by detecting the maximum peak position of the crosscorrelation signal. To improve the resolution, a polynomial fit was applied around this maximum peak [340].

\subsubsection{Magnetic hyperthermia}

The interaction between radiofrequency magnetic field and magnetic nanoparticles results in the conversion of electromagnetic energy into thermal energy. The power dissipated during this interaction for a relatively low applied magnetic field can be expressed as [239, 271]:

$$
P=\frac{1}{2} \mu_{0} \chi_{0} \omega H^{2} \frac{\omega \tau}{1+(\omega \tau)^{2}}
$$

where $\chi_{0}$ is the static susceptibility and $\tau$ is effective relaxation time. To conduct the hyperthermia procedure, the coil was supplied using a magnetic hyperthermia system. The magnetic hyperthermia system was basically composed of an AC power supply, a rectifier circuit, a cross-coupled inverter, and a parallel resonant tank circuit (with a variable capacitor bank). The inverter was designed so that the resonant frequency of the tank circuit is directly followed, allowing a great variety of operating frequencies. Due to this reason, the system is versatile in terms of providing the possibility to be used with different coil formats. The details about the design, construction and characterization of the magnetic hyperthermia system are described elsewhere [92]. In the present study, the capacitance $1200 \mathrm{nF}$ in the resonant tank was chosen, resulting in the operating frequency $106 \mathrm{kHz}$. For the present study, the experiments were conducted at 7.5 and $9.2 \mathrm{kA} / \mathrm{m}$ field amplitudes.

\subsubsection{Ultrasound thermal strain imaging}

Beamformed RF data were acquired, at a $40 \mathrm{MHz}$ sampling frequency, during the magnetic hyperthermia procedure using a $0.5 \mathrm{~Hz}$ frame rate. The data were assessed using the SonixRP 
clinical imaging software in research mode. The acoustic transmission beam was focused at $70 \mathrm{~mm}$, where the inclusion was located (for more details about the tissue-mimicking phantom, see section 2.5). A total 50 frames were obtained during 100 seconds. Three frames were acquired before turning the magnetic hyperthermia system on and the system was ON only for 62 seconds.

Since a water-based phantom was used, the speed of sound (c) increased with temperature similarly to what is observed for water-bearing tissues [341]. For a limited temperature range $\left(\Delta T<10{ }^{\circ} \mathrm{C}\right)$, temperatures below $50{ }^{\circ} \mathrm{C}$, and assuming a nonfatty tissue [342], this relation can be considered linear as follows:

$$
\frac{\partial c}{\partial T}=\beta c\left(T_{0}\right)
$$

where $\beta$ is the linear coefficient relating the variation in the speed of sound to temperature and $T_{0}$ is the initial temperature at ROI. In this case, the apparent position of the scatterers within the phantom moves towards the transducer due to temperature rise and a consequent time shift $\Delta t(z)$ in the backscattered waves. The variation in temperature along depth $\Delta T(z)$ can be written as [343]:

$$
\Delta T(z)=\frac{c\left(T_{0}\right)}{2}\left(\frac{1}{\alpha-\beta}\right) \frac{\partial}{\partial z}[\Delta t(z)]
$$

where $\alpha$ is the linear coefficient of thermal expansion and the term $(\partial / \partial z)[\Delta t(z)]$ is known as the thermal strain. The coefficient $k=1 /(\alpha-\beta)$ is dependent on the material and can be experimentally determined. In the present study, $k$ and $c\left(T_{0}\right)$ were assumed to be constant independent of the location being analyzed within the phantom.

The same speckle tracking strategy, as described in the previous section for detecting the displacement in MMUS images, was used here to estimate the apparent displacement due to the variation in temperature. Axial strain was calculated using linear regression windows to estimate the gradient of the apparent displacement map [344]. Thermal strain images were obtained by transforming the axial strains into temperature variation by applying the calibration factor. A fiber optic thermometer system (Qualitrol NOMAD-Touch Portable Fiber Optic Monitor) was used to record the temperature variation during the application of the magnetic field. The temperature sensor was place in the middle of the inclusion such that it could be 
visualized in the B-mode image. By comparing the temperature measured by the thermometer with the thermal strain estimated in a ROI positioned beside the sensor, the calibration factor was calculated.

\subsubsection{Magnetic nanoparticles and phantom preparation}

Magnetic nanoparticles used in this study were zinc-substituted magnetite nanoparticles with nominal formula $\mathrm{Zn}_{0.1} \mathrm{Fe}_{0.9} \mathrm{Fe}_{2} \mathrm{O}_{4}$. This composition was prepared using coprecipitation method and the resultant particles had the mean diameter $12.4 \pm 2 \mathrm{~nm}$ and $\mathrm{M}_{\mathrm{s}}=83 \mathrm{kA} \cdot \mathrm{m}^{2} / \mathrm{kg}$. Details about the preparation and characterization of the nanoparticles and their performance in MMUS imaging and hyperthermia experiments have been reported elsewhere [90, 93].

A cubic gelatin/agar phantom with a cylindrical inclusion containing magnetic nanoparticles was manufactured. The inclusion and background were prepared using a solution consisted of 4 wt. \% gelatin (Gelita, Sao Paulo, Brazil) and 2 wt. \% agar (Bacteriologic CAT. RM026 supplied by Himedia). The procedure to make the phantom materials was similar to the description in $[187,188]$. The solution was heated to $90^{\circ} \mathrm{C}$ and then rested in room temperature to reach $35{ }^{\circ} \mathrm{C}$ while gentle mixing. At this temperature, formaldehyde was added to the solution with mass equal to $5 \%$ of the mass of gelatin. In the case of the inclusion, aqueous colloidal dispersion of the magnetic nanoparticles with concentration $5 \mathrm{wt}$. $\%$ was also added to the solution at $35{ }^{\circ} \mathrm{C}$ as the final concentration $2 \mathrm{wt} . \%\left(\sim 20 \mathrm{mg} / \mathrm{cm}^{3}\right)$ for magnetic nanoparticles was reached. The mixture was poured in the molds and kept inside a refrigerator for 24 hours. The inclusion was produced using a cylindrical mold with $16 \mathrm{~mm}$ in diameter and $7 \mathrm{~mm}$ in height and for the background a cubic mold with $75 \mathrm{~mm} \times 75 \mathrm{~mm} \times 75 \mathrm{~mm}$ was used.

\subsection{Results and discussion}

\subsubsection{MMUS imaging}

Since the phantom was positioned such that the inclusion was aligned with the center of the coil, the magnetic field strength was measured on the central axis of the coil, $2 \mathrm{~mm}$ away from its surface. Figure 6.2(a) shows the measurements for charging voltages 30, 40, and $50 \mathrm{~V}$, resulting in maximum magnetic field of 235,325 , and $410 \mathrm{kA} / \mathrm{m}$, respectively. These $\sim 2 \mathrm{~ms}$ magnetic pulses induced sufficient displacements to generate high quality MMUS images. As it was expected (see equation (6.1)), the induced displacements were directly proportional to the amplitude of the applied magnetic field (figure 6.2(b)). 


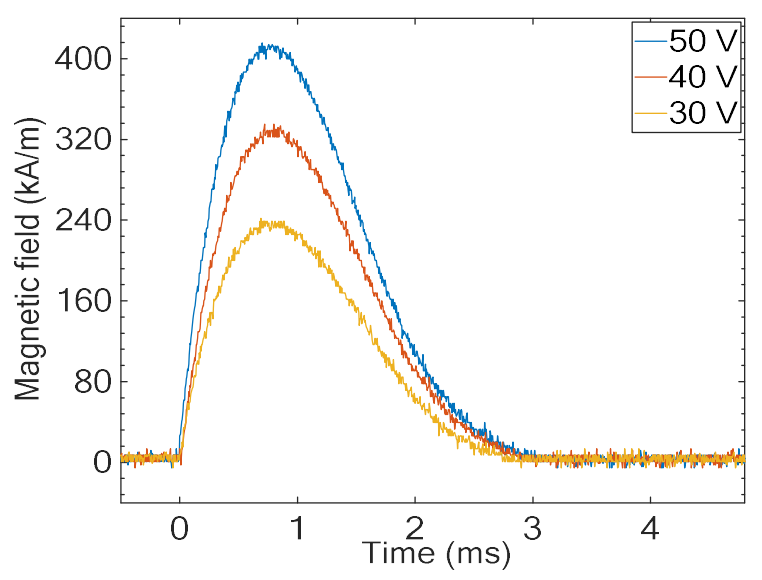

(a)

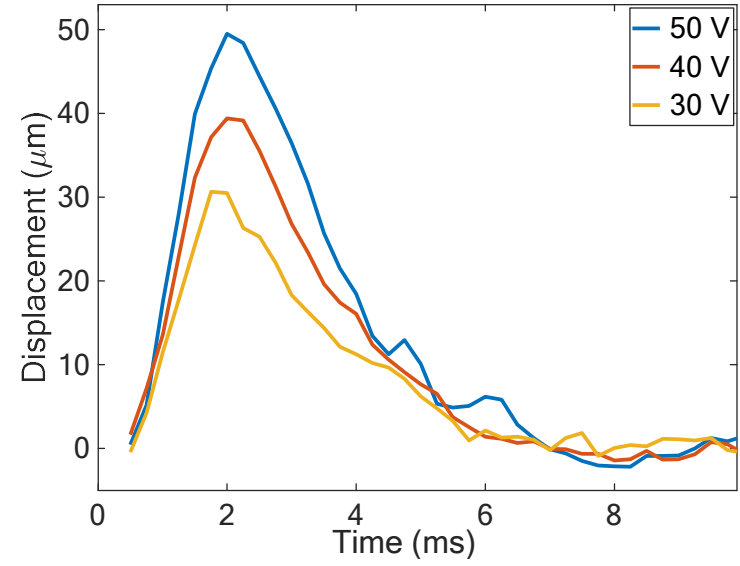

(b)

Figure 6.2 a) Magnetic field strength on the central axis of the coil, $2 \mathrm{~mm}$ away from its surface. b) Induced displacements measured in the center of the inclusion.

Figure 6.3(a) shows the B-mode image of the phantom using the RF data obtained using unfocused plane waves emission. Figure 6.3(b) shows a peak displacement image, obtained independent of the time to peak, for the magnetic field amplitude $325 \mathrm{kA} / \mathrm{m}$. In this image, higher displacements $(>35 \mu \mathrm{m})$ clearly show the location of the inclusion. Lower displacements, outside the inclusion, are due to the propagation of the shear and surface waves. This observation can immediately be understood from the time to peak displacement images shown in figures 6.3(c) and (d), for $t<3 \mathrm{~ms}$ and $t<6.5 \mathrm{~ms}$, respectively. These images show that the time to peak displacement within the inclusion was consistently higher than $2 \mathrm{~ms}$ and lower than $3 \mathrm{~ms}$. On the other hand, the time to peak increased with distance from the inclusion, which is the result of elastic wave propagation. The speed of the shear wave estimated here was $4.5 \mathrm{~ms}^{-1}$. In addition to localizing magnetic nanoparticles, MMUS can provide useful information regarding the viscoelastic properties of the medium by monitoring the shear waves generated from the motion induced to magnetic nanoparticles [47]. In the case of the hyperthermia application, for example, thermal induced viscoelastic changes in the tissue after the treatment can be used as a tool to study the thermal dose delivered to the tissue. Huang et al [325] used a different method (magnetomotive optical coherence elastography) to monitor these changes and estimated the thermal dose after the hyperthermia procedure. 


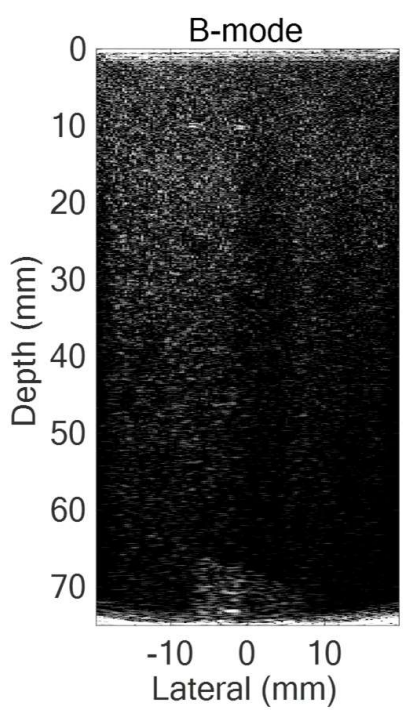

(a)

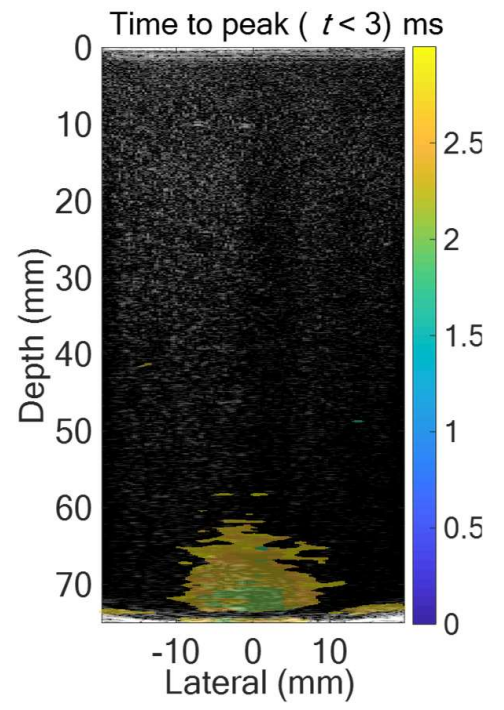

(c)

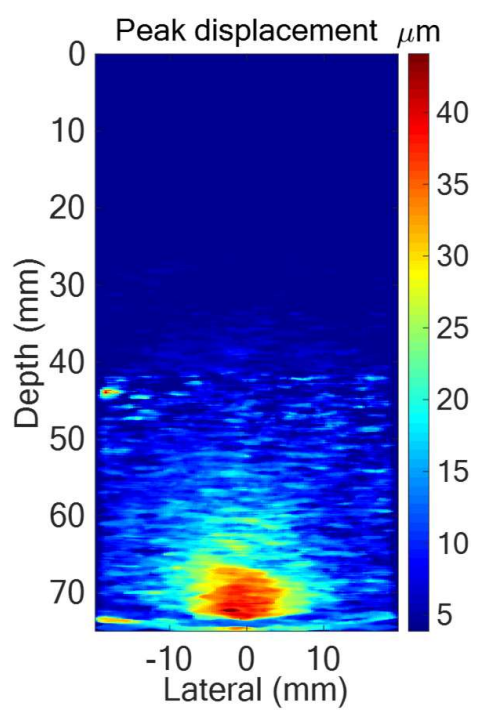

(b)

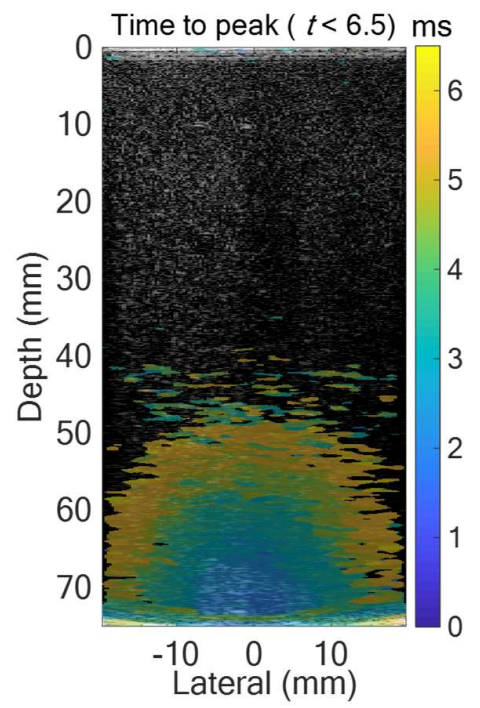

(d)

Figure 6.3 a) B-mode image of the phantom acquired using unfocused plane waves emission. b) MMUS peak displacement image; $c$ ) and d) show images of the time to reach the peak displacements for $\mathrm{t}<3$ and $\mathrm{t}<6.5 \mathrm{~ms}$, respectively.

Figure 6.4 shows the MMUS peak displacements overlaid on the B-mode image. We believe the displacements are higher in regions close to the surface and in the center of the inclusion because of higher magnetic field magnitudes close to the center of the coil. To obtain this final MMUS image, the time to peak shown in figure 6.3(c) was converted into a binary mask and was used to eliminate the peak displacements outside the inclusion. In order to assess the accuracy of MMUS in localizing magnetic nanoparticles, the MMUS image was compared with the B-mode image. The comparison was possible because the inclusion borders were well 
defined in the B-mode images. The results showed the accuracy of $85 \%$, determined by the sum of the percentage of false positive and false negative MMUS pixels.

Ultrasound spatial resolution and contrast are not sufficient for the directly visualization of magnetic nanoparticles within tissues. On the other hand, MMUS overcome this limitation by indirectly detecting the magnetic nanoparticle-laden regions. Therefore, MMUS is a real time and low cost technique to map magnetic nanoparticle distribution within tissue. The sensitivity of this technique depends on various parameters including mechanical properties of tissue, magnetic field parameters, and intrinsic properties of magnetic nanoparticles. In [345], the authors show that for magnetic nanoparticles with typical $M_{\mathrm{s}}$ of $50 \mathrm{~A} \cdot \mathrm{m}^{2} / \mathrm{kg}$, detectable motion can be observed at concentrations as low as $0.04 \mathrm{mg} / \mathrm{cm}^{3}$. Improvement in SNR for further enhancing this concentration threshold can potentially be achieved by tuning the intrinsic properties of the magnetic nanoparticles $[48,90]$.

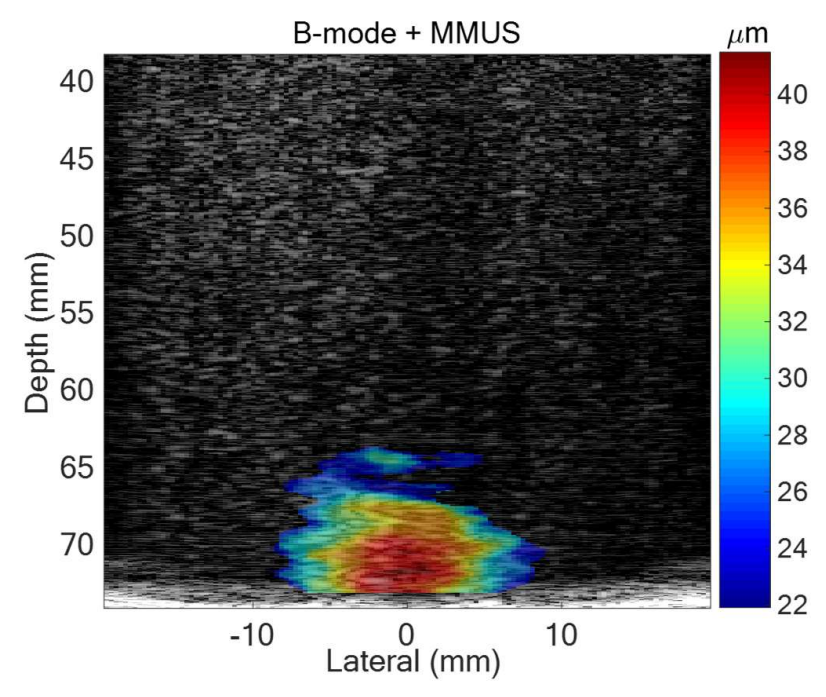

Figure 6.4 The final MMUS image overlaid on the B-mode image.

\subsubsection{Ultrasound thermal strain imaging}

After acquiring the MMUS images, the power supply of the coil was switched to the magnetic hyperthermia system to generate the radiofrequency magnetic field. Two magnetic field amplitudes, 7.5 and $9.2 \mathrm{kA} / \mathrm{m}$ with frequency $106 \mathrm{kHz}$, were examined. Prior to starting the magnetic hyperthermia procedure, the fiber optic sensor was placed in the middle of the inclusion by approaching from the bottom side of the phantom and through the center of the 
coil (see figure 6.1); such that the sensor was within the transducer field of view. This procedure enhanced the accuracy of the calibration factor for thermal images. The B-mode image shown in figure 6.5(a) was acquired using the SonixRP clinical imaging software.

Figure 6.5(b) shows an example of the apparent displacement map of the phantom exposed to the field amplitude $7.5 \mathrm{kA} / \mathrm{m}$, where, due to the local temperature elevation and assumption of constant speed of sound for the entire medium, ultrasound echoes were shifted in time. By applying the axial gradient on this image, the thermal strain map, $(\partial / \partial z)[\Delta t(z)]$, was also calculated (figure 6.5(c)). As it can clearly be seen, both the apparent displacements and the thermal strain are more evident in the region where the inclusion is located. Using the temperature variation recorded by the fiber optic thermometer and the estimated strain from the $\mathrm{ROI}_{\mathrm{T}}$ positioned right next to the temperature sensor, the calibration factor was obtained. It is interesting to observe that the temperature sensor cannot be visualized in the B-mode image; however, the induced signal decorrelation and a consequent error during the speckle tracking procedure, which can be visualized in the middle of the inclusion in both apparent displacement and thermal strain images, indicated the location of the sensor. For the phantom used in this study, the calibration factor was estimated as $k=(-995 \pm 20){ }^{\circ} \mathrm{C}$; which is in accordance with other studies in the literature [324, 346].

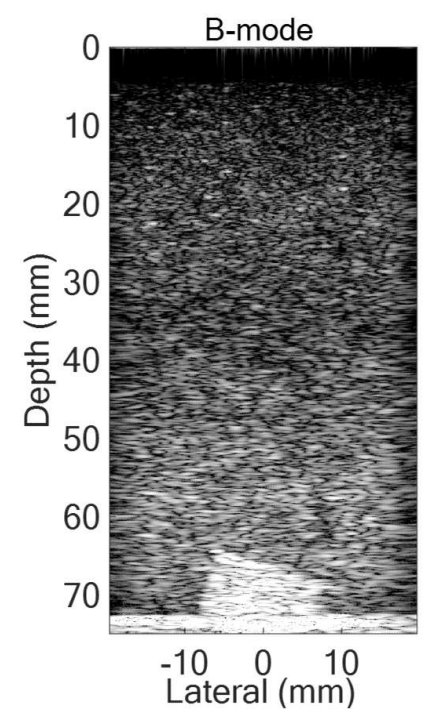

(a)

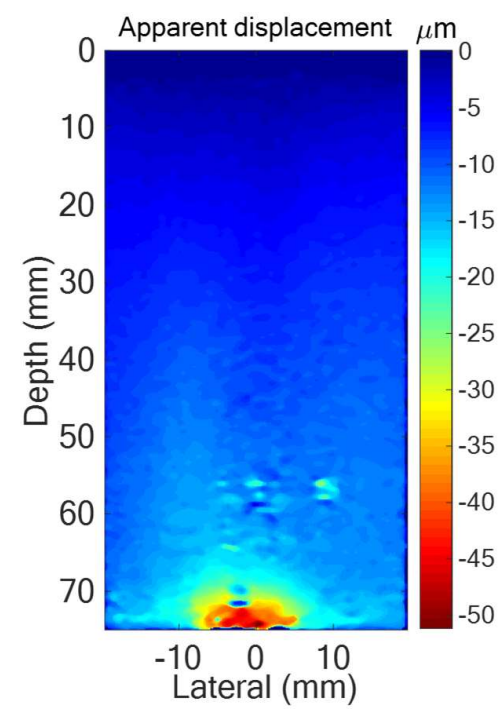

(b)

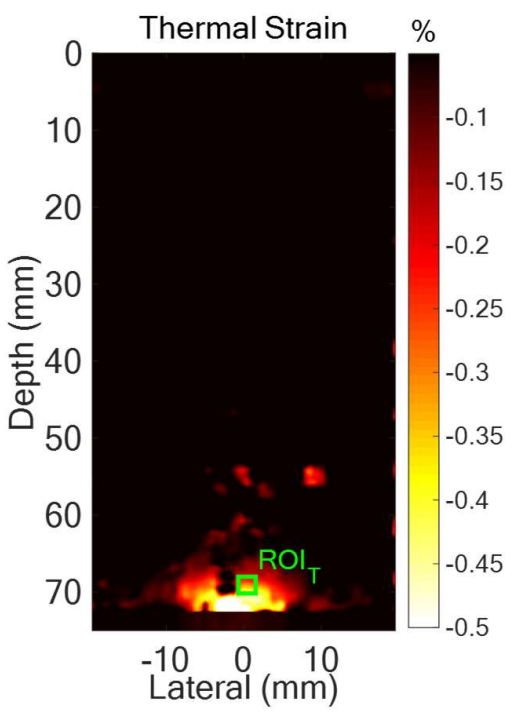

(c)

Figure 6.5 a) B-mode image obtained using the SonixRP clinical imaging software, b) an example of the apparent displacement map, and c) its thermal strain map at the field amplitude $7.5 \mathrm{kA} / \mathrm{m}$. 
The thermal images were obtained by applying the calibration factor to the estimated thermal strains. Figure 6.6 illustrates three examples of thermal images, for applied field $9.2 \mathrm{kA} / \mathrm{m}$, combined with the B-mode images acquired with the SonixRP clinical imaging software. These images showed the temperature rise was confined to the inclusion for the first 28 seconds and then diffused to the background. In the background, less than $3{ }^{\circ} \mathrm{C}$ temperature variation was observed during the entire experiment. Therefore, temperature mapping with good spatial and temporal resolution can be achieved by this method during the treatment procedure. Real-time temperature monitoring is of crucial importance for thermotherapy methods in general. The rationale behind the thermotherapy routines, especially in magnetic hyperthermia, is to selectively deliver a sufficient thermal dose to the tumor site. However, uncontrolled temperature rise may also damage the surrounding healthy tissues. Particularly, due to the tissue heterogeneity and different blood perfusion arising from changes in tissue architecture, the delivered thermal dose may vary significantly [347]. Therefore, the real-time monitoring feature in ultrasound thermometry can considerably enhance the efficiency of the magnetic hyperthermia technique.
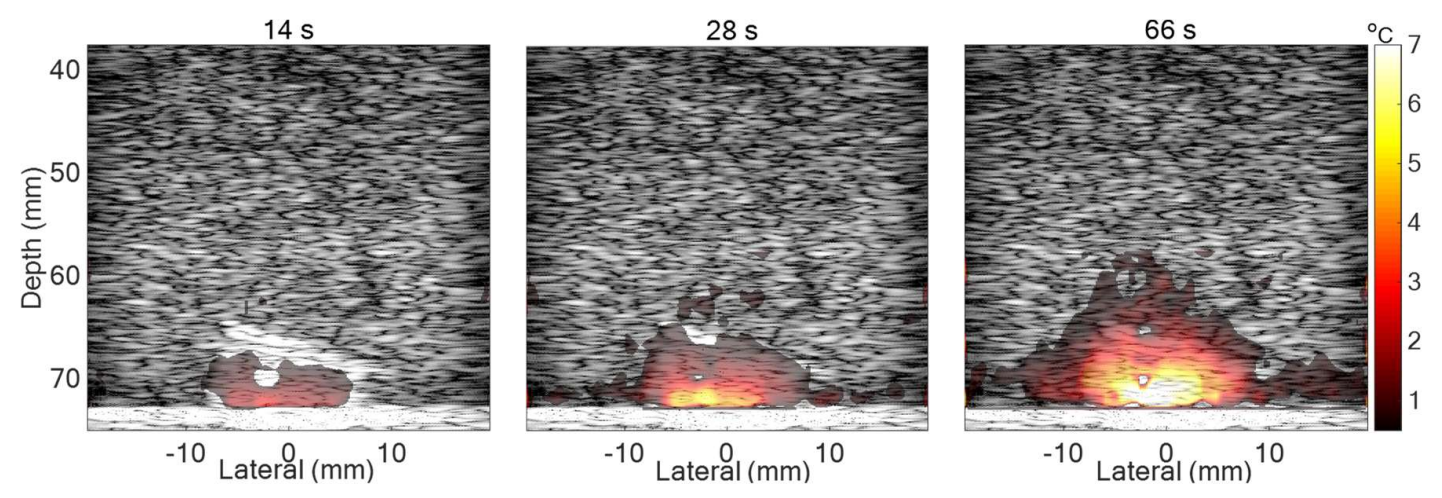

Figure 6.6 Thermal images obtained 14, 28, $66 \mathrm{~s}$ after starting the hyperthermia procedure using the magnetic field strength of $9.2 \mathrm{kA} / \mathrm{m}$.

Figure 6.7 compares the temperature measurements for the entire hyperthermia experiments; the dashed lines are the temperature measured with the fiber optic thermometer and the hollow symbols are the results from the ultrasound thermometry. In both cases, substantial concordance $\left(\rho_{\mathrm{c}}>0.97\right)$ between the results was verified. This method has advantages when comparing it, for example, with infrared thermography which has been wieldy used as a noninvasive method for monitoring the temperature during magnetic hyperthermia in different 
experimental conditions [326, 348-351]. The main disadvantage of the infrared thermography is that it is limited only to superficial temperature measurements, hence, limiting its applicability to most of the realistic in vivo experiments where the tumor is located in internal organs. Moreover, it is strongly angle dependent, where errors as high as $7{ }^{\circ} \mathrm{C}$ may happen by choosing a wrong setup [352]. Lastly, the difference between ambient and surface temperatures can be a limitation of this method. However, recently Rodrigues et al. [352] proposed a model to overcome this limitation.

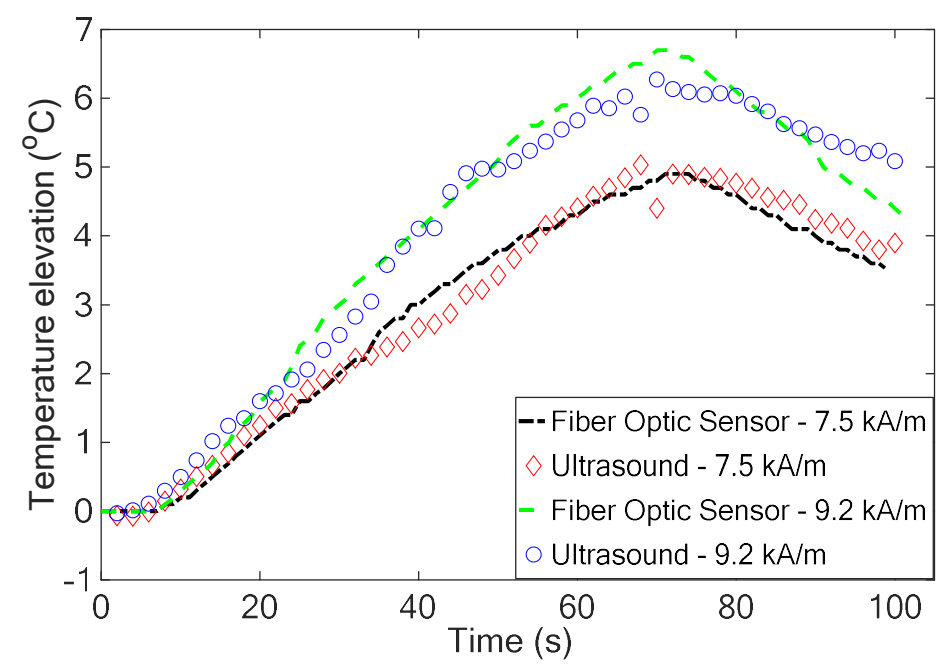

Figure 6.7 Comparison between the temperature measurement using the fiber optic thermometer and ultrasound thermal imaging.

Despite the fact that excellent temperature maps can be obtained by MR thermometry [318], the existence of the strong static magnetic field inhibit the integration of this method with magnetic hyperthermia. The static magnetic field can substantially influence the heating efficiency of magnetic nanoparticles [353, 354]. Therefore, ultrasound thermometry, which has been used in other hyperthermia methods [331, 332, 334], can be currently considered as a powerful and feasible tool to monitor the temperature distribution at considerably deep ROIs during magnetic hyperthermia. It should, however, be noted that the present phantom study is a preliminary proof-of-concept of the integration of ultrasound thermometry with magnetic hyperthermia. This method, especially for in vivo experiments, has certain limitations [342], which need to be addressed for further application in magnetic hyperthermia. Its sensitivity to the unavoidable motions, due to breathing and artery pulsation, during the experiment can impose significant artifacts to the thermal images as in MR thermometry [318]. Several methods have been proposed to overcome this limitation [324, 355-357], which will be the 
subject of a future study in our group for the system proposed in the present paper. Another source of error is aberration of the ultrasound wave due to local changes in sound speed, appearing as ripples behind the heated region [343]. However, several aberration correction methods have been introduced to reduce this error [324, 343, 346]. Finally, a common but fundamental consideration in any thermometry technique is determining the calibration factor, which has to be considered in ultrasound thermometry due to the heterogeneity of the tissues.

6.3.3. Comparing temperature elevation during hyperthermia and induced displacements during MMUS

The induced displacement in MMUS and the local temperature rise in magnetic hyperthermia are proportional to the concentration of magnetic nanoparticles. Therefore, it is expected that for regions with higher displacements, higher temperatures would also be achieved. This hypothesis is especially relevant in our study because the spatial distribution of the magnetic field profile in both experiments (MMUS and hyperthermia) is the same (same coil in both cases). To this end, for ROI within the inclusion $(6.2 \mathrm{~mm} \times 7.4 \mathrm{~mm})$, the temperature variation (heating rate) was plotted against the corresponding peak displacement estimated in the same spatial position. More specifically, the heating rate was considered as the linear slope of the temperature versus time in the interval $30-60 \mathrm{~s}$ after starting the hyperthermia procedure. Figure 6.8 shows the result of the heating rate versus peak displacements. Clearly, regions with higher displacements had more pronounced temperature elevation independent of their spatial position, showing a linear relationship between these two parameters with Pearson correlation $\mathrm{r}=0.92$.

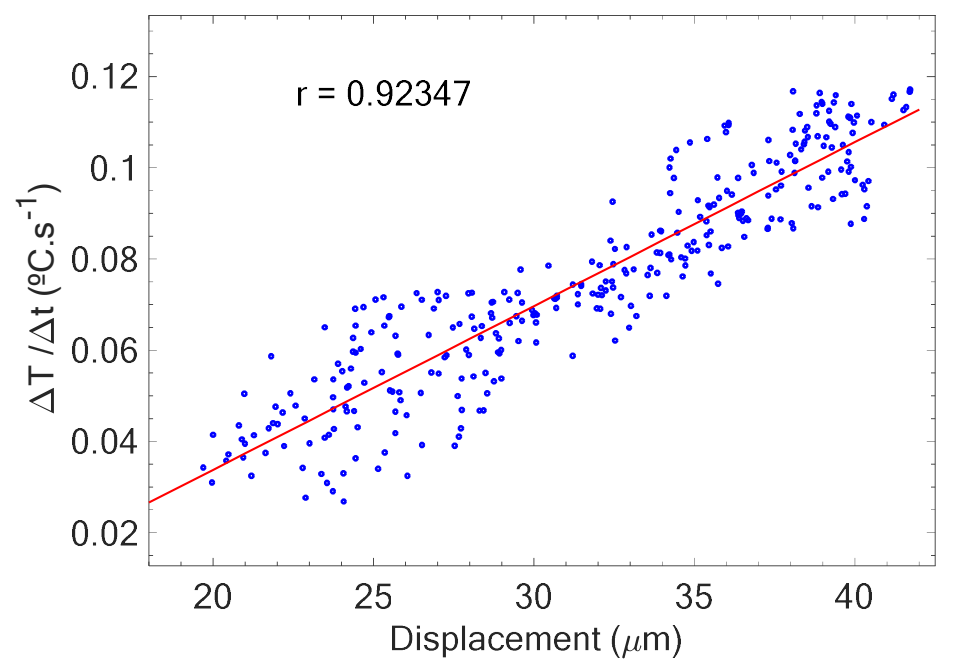

Figure 6.8 Correlation between the temperature rise (heating rate) and the observed displacement independent of the spatial position. 
Since the heating rate and magnitude of the induced displacement are dependent on either the strength of the magnetic field or nanoparticles concentration at a specific location, acquiring the MMUS images prior to the hyperthermia procedure, may provide a rough estimation of temperature distribution over the region exposed to the magnetic fields. This observation is valid only if the spatial distribution of the applied magnetic field for both experiments is the same, which is the case in the system proposed in this study. Equation (6.1) shows that displacement is dependent on the magnitude of applied magnetic field $(H)$ and its gradient, while according to equation (6.2), the dissipated power (appeared in the form of heat) is proportional to $H^{2}$. However, drawing a conclusion about the exact relation between the induced displacement and the heating rate is not very straightforward and more considerations must be taken into account. For example, the displacement magnitude depends not only on the strength of the magnetic field but also on its gradient and on the elasticity of tissues. In addition, for in vivo experiments this comparison can be more complicated due to the complex structure of the tissues.

Temperature monitoring in clinical magnetic hyperthermia routine is a very complex task. For example, in two reports related to prostate cancer treatment $[358,359]$, the heat distribution within the tumor sites was estimated from numerical calculation using information about local temperature from sensors invasively positioned within the prostate and nanoparticles concentration in the tissue obtained from computed tomography images. Then, using the known specific absorption rate (SAR) values of magnetic nanoparticles, the strength of the magnetic field during treatment, assuming a constant perfusion, and solving the bio-heat transfer equation the temperature distribution was estimated. The invasive temperature measurements were used as reference to fit the results of the temperature distribution map.

Recently, a robust theranostic platform combining MPI and magnetic hyperthermia was introduced for arbitrary localization of magnetic hyperthermia therapy. They first reported the system with phantom [77] and later in an in vivo study [78]. This system can effectively image the biodistribution of the magnetic nanoparticles and then apply a localized $354 \mathrm{kHz}$ magnetic field for the hyperthermia procedure. The temperature monitoring in their system was conducted using a fiber optic probe. They correlated the initial temperature rise $(\mathrm{dT} / \mathrm{dt})$ to the MPI image intensity, which is linearly proportional to the mass of magnetic nanoparticles. They found a linear correlation between the MPI pixel intensity and the initial temperature rise; therefore, concluding MPI image as an invaluable tool for thermal dose planning. 
Although the system proposed in the present study was evaluated with a phantom experiment, which is far from realistic conditions, it may open a new horizon for magnetic hyperthermia planning, owing to its simplicity, low-cost, real-time, and non-invasive features. To the best of our knowledge, this is the first theranostic platform using a single coil for both magnetic hyperthermia and to detect the magnetic nanoparticles. It has, inherently, the potential of being independent of any other modalities for diagnosis, by acquiring B-mode, Doppler and molecular images or by examining the viscoelastic properties of the tissue; and for therapy (magnetic hyperthermia), while monitoring the temperature during treatment and the response to the therapy through the viscoelastic information or by acquiring molecular images. Currently, the only treatment planning and temperature monitoring method available for deep tissues in clinical [360] and small animal hyperthermia [78] is to predict the delivered thermal dose based on the information about magnetic nanoparticles, applied magnetic field, and estimated tissue perfusion. Comparing with [78], the system presented in this study has two advantages. First, it addresses the issue of the non-invasive real-time temperature monitoring during magnetic hyperthermia through a nonionizing vastly available imaging technique. Second, it is simpler and lower cost technique, as the cost and complexity of a MPI system would be comparable with a clinical MRI scanner.

\subsection{Summary}

The present report was a phantom study to demonstrate the feasibility of integrating magnetomotive and thermal ultrasound imaging with magnetic hyperthermia as a theranostic

platform. The idea of using nanotheranostic agent was also examined using a single magnetic nanoparticles composition, which served as contrast agent in MMUS and as heating mediator. The system proposed here, in addition to localizing magnetic nanoparticles, can successfully provide real-time two-dimensional temperature maps during the magnetic hyperthermia procedure. This temperature map can be obtained for any depth within the limitation of the ultrasound imaging devices. Moreover, it was demonstrated that acquiring MMUS images prior to magnetic hyperthermia procedure can qualitatively predict the temperature distribution of the region containing the magnetic nanoparticles. Therefore, this system has the potential to be used independent of any other modalities (at least for preclinical studies) for diagnosis, treatment and monitor the effect of the therapy through viscoelastic properties of the tissues or molecular imaging. 


\section{Chapter 7 : Conclusion and future works}

This thesis represent a systematic study on the theranostic potential of zinc substituted magnetite nanoparticles $\left(\mathrm{Zn}_{\mathrm{x}} \mathrm{Fe}_{1-\mathrm{x}} \mathrm{Fe}_{2} \mathrm{O}_{4}, \mathrm{x}=0.1,0.2,0.3,0.4\right)$. A simple and low cost coprecipitation route was used to synthesize the nanoparticles and the resultant particles were in superparamagnetic state at room temperature. Structural and magnetic characterization of the samples showed that incorporation of zinc in magnetite structure can remarkably enhance its saturation magnetization while maintaining its cubic spinel structure. It has been proven that saturation magnetization is a key factor in several biomedical applications. The prepared nanoparticles were evaluated as contrast agent for MMUS imaging (the diagnostic application) in phantom studies. Sample $\mathrm{x}=0.1$ with enhanced saturation magnetization showed more than twofold increase in observed displacement compared to magnetite. This enhancement can potentially result in decreasing the minimum dosage of the magnetic nanoparticles necessary to generate the signal for acquiring MMUS images.

To investigate the potential of the samples as heating mediators for magnetic hyperthermia, a versatile magnetic hyperthermia system capable of generating magnetic fields up to $27.2 \mathrm{kA} / \mathrm{m}$ in a wide range of operation frequencies was designed and constructed. This low cost, frequency adjustable, and highly stable system was a modification of the Mazzilli inverter.

Next, the magnetic hyperthermia study was performed in a wide range of experimental conditions. All the experiments were conducted in linear regime and respecting the threshold proposed for major discomfort in the patient i.e. the product $H_{0} f \leq 5 \times 10^{9} \mathrm{~A} \cdot \mathrm{m}^{-1} \cdot \mathrm{s}^{-1}$. Due to different intrinsic properties, the prepared nanoparticles showed to be a very useful tool to observe the effect of parameters such as saturation magnetization, anisotropy and size on the heating efficiency of the magnetic nanoparticles. Intrinsic properties of the nanoparticles had diverse effects related to the dipolar interactions on their heating efficiency. For linear regime, this study resulted in two main conclusions: i) at the same sample concentration the strength of dipolar interactions is determined by the intrinsic properties; ii) if the dipolar interactions are strong enough to affect the heating efficiency, the parameter $\sigma=K_{\text {eff }} V / k_{\mathrm{B}} T$ determines the type of the effect. As interactions increase the parameter $\sigma$, for any $\sigma$ value resulting in $\omega \tau<1$, interactions can increase the heating efficiency. On the other hand, for $\omega \tau \geq 1$ interactions have the opposite effect. This shows also the importance of choosing the appropriate frequency to increase the heating efficiency of high interacting magnetic nanoparticle systems. Moreover, 
the sample $\mathrm{Zn}_{0.1} \mathrm{Fe}_{0.9} \mathrm{Fe}_{2} \mathrm{O}_{4}$ again had a superior performance compared to the other samples, offering a great potential to be considered as a theranostic agent.

Finally, a theranostic platform was proposed to integrate magnetomotive and thermal ultrasound imaging with magnetic hyperthermia. This system in addition to localizing magnetic nanoparticles, can successfully provide real-time two-dimensional temperature maps during the magnetic hyperthermia procedure. To the best of our knowledge, this was the first study on using ultrasound thermometry to monitor the temperature during magnetic hyperthermia. The unique feature of this platform is that the diagnostic and therapeutic applications are achieved through a single coil and a single magnetic nanoparticle composition $\left(\mathrm{Zn}_{0.1} \mathrm{Fe}_{0.9} \mathrm{Fe}_{2} \mathrm{O}_{4}\right.$, $)$, which enables the entire system to be used as a theranostic platform.

A proposal for future work based on the results presented in this thesis can be divided in two categories:

i) Since the rationale behind magnetic hyperthermia is to selectively deliver a sufficient thermal dose to the tumor site, targeted magnetic hyperthermia can significantly enhance the specificity of the therapy by sufficient accumulation and adequate distribution of the magnetic nanoparticles in the target cells. Functionalizing the optimized magnetic nanoparticles by conjugating them with targeting ligands, such as aptamers or peptides, that bind to cell receptors highly expressed in tumor cells can be a proposal for future work.

ii) The theranostic platform proposed in this study was evaluated in a phantom study which is far from realistic conditions for in vivo experiments, where unavoidable motions, due to breathing and artery pulsation during the experiment can impose significant artifacts to the thermal images. Therefore, examining the system for in vivo experiment is of crucial importance. 


\section{References}

[1] L. Zhu, Z. Zhou, H. Mao, L. Yang, Magnetic nanoparticles for precision oncology: theranostic magnetic iron oxide nanoparticles for image-guided and targeted cancer therapy, Nanomedicine, 12 (2016) 73-87.

[2] C.P. Wild, The global cancer burden: necessity is the mother of prevention, Nature Reviews Cancer, 19 (2019) 123-124.

[3] WHO, Cancer Key Statistics, in, https://www.who.int/cancer/resources/keyfacts/en/, 2019.

[4] F. Bray, J. Ferlay, I. Soerjomataram, R.L. Siegel, L.A. Torre, A. Jemal, Global cancer statistics 2018: GLOBOCAN estimates of incidence and mortality worldwide for 36 cancers in 185 countries, CA: A Cancer Journal for Clinicians, 68 (2018) 394-424.

[5] Z. Xiao, O.C. Farokhzad, Aptamer-Functionalized Nanoparticles for Medical Applications: Challenges and Opportunities, ACS Nano, 6 (2012) 3670-3676.

[6] N. Ahmed, H. Fessi, A. Elaissari, Theranostic applications of nanoparticles in cancer, Drug Discovery Today, 17 (2012) 928-934.

[7] H. Jo, C. Ban, Aptamer-nanoparticle complexes as powerful diagnostic and therapeutic tools, Experimental \&Amp; Molecular Medicine, 48 (2016) e230.

[8] G. Kandasamy, D. Maity, Recent advances in superparamagnetic iron oxide nanoparticles (SPIONs) for in vitro and in vivo cancer nanotheranostics, Int. J. Pharm., 496 (2015) 191-218.

[9] O.C. Farokhzad, R. Langer, Impact of Nanotechnology on Drug Delivery, ACS Nano, 3 (2009) 16-20. [10] J.E. Rosen, L. Chan, D.-B. Shieh, F.X. Gu, Iron oxide nanoparticles for targeted cancer imaging and diagnostics, Nanomed. Nanotechnol. Biol. Med., 8 (2012) 275-290.

[11] J.D.G. Durán, J.L. Arias, V. Gallardo, A.V. Delgado, Magnetic Colloids As Drug Vehicles, J. Pharm. Sci., 97 (2008) 2948-2983.

[12] S. Bamrungsap, T. Chen, M.I. Shukoor, Z. Chen, K. Sefah, Y. Chen, W. Tan, Pattern Recognition of Cancer Cells Using Aptamer-Conjugated Magnetic Nanoparticles, ACS Nano, 6 (2012) 3974-3981.

[13] L. Borlido, A.M. Azevedo, A.C.A. Roque, M.R. Aires-Barros, Magnetic separations in biotechnology, Biotechnol. Adv., 31 (2013) 1374-1385.

[14] J.M. Perez, L. Josephson, T. O'Loughlin, D. Högemann, R. Weissleder, Magnetic relaxation switches capable of sensing molecular interactions, Nat. Biotechnol., 20 (2002) 816-820.

[15] G.C. Papaefthymiou, Nanoparticle magnetism, Nano Today, 4 (2009) 438-447.

[16] P. Pradhan, J. Giri, G. Samanta, H.D. Sarma, K.P. Mishra, J. Bellare, R. Banerjee, D. Bahadur, Comparative evaluation of heating ability and biocompatibility of different ferrite-based magnetic fluids for hyperthermia application, Journal of Biomedical Materials Research Part B: Applied Biomaterials, 81B (2007) 12-22.

[17] L.H. Reddy, J.L. Arias, J. Nicolas, P. Couvreur, Magnetic Nanoparticles: Design and Characterization, Toxicity and Biocompatibility, Pharmaceutical and Biomedical Applications, Chem. Rev., 112 (2012) 5818-5878.

[18] B.D. Cullity, C.D. Graham, Introduction to Magnetic Materials, Second Edition ed., John Wiley \& Sons, Inc., New Jersey, 2009.

[19] A. Goldman, Handbook of Modern Ferromagnetic Materials, Springer US, 2012.

[20] M. Reis, Fundamentals of magnetism, Elsevier, 2013.

[21] A.H. Morrish, The Physical Principles of Magnetism, IEEE Press, New York, 2001.

[22] R.C. O'Handley, Modern Magnetic Materials: Principles and Applications, John Wiley \& Sons, New York, 1999.

[23] A.P. Guimaraes, Principles of Nanomagnetism, Springer, New York, 2009.

[24] O. Petracic, Superparamagnetic nanoparticle ensembles, Superlattices and Microstructures, 47 (2010) 569-578.

[25] C. Buzea, I.I. Pacheco, K. Robbie, Nanomaterials and nanoparticles: Sources and toxicity, Biointerphases, 2 (2007) MR17-MR71.

[26] C.P. Bean, J.D. Livingston, Superparamagnetism, Journal of Applied Physics, 30 (1959) S120-S129. 
[27] C.P. Bean, I.S. Jacobs, Magnetic Granulometry and Super-Paramagnetism, Journal of Applied Physics, 27 (1956) 1448-1452.

[28] C.G. Ramankutty, S. Sugunan, Surface properties and catalytic activity of ferrospinels of nickel, cobalt and copper, prepared by soft chemical methods, Applied Catalysis A: General, 218 (2001) 3951.

[29] A. Joseph, S. Mathew, Ferrofluids: Synthetic Strategies, Stabilization, Physicochemical Features, Characterization, and Applications, ChemPlusChem, 79 (2014) 1382-1420.

[30] E. Betzig, J.K. Trautman, R. Wolfe, E.M. Gyorgy, P.L. Finn, M.H. Kryder, C.H. Chang, Near-Field Magneto-Optics and High Density Storage, Opt. Photon. News, 3 (1992) 24-25.

[31] I. Koh, L. Josephson, Magnetic Nanoparticle Sensors, Sensors, 9 (2009).

[32] J.B. Weaver, A.M. Rauwerdink, E.W. Hansen, Magnetic nanoparticle temperature estimation, Medical Physics, 36 (2009) 1822-1829.

[33] A.M. Rauwerdink, J.B. Weaver, Viscous effects on nanoparticle magnetization harmonics, Journal of Magnetism and Magnetic Materials, 322 (2010) 609-613.

[34] J.B. Weaver, K.M. Rauwerdink, A.M. Rauwerdink, I.M. Perreard, Magnetic spectroscopy of nanoparticle Brownian motion measurement of microenvironment matrix rigidity, Biomed. Tech./Biomed. Eng., 58 (2013) 547-550.

[35] L.F. Zanini, N.M. Dempsey, D. Givord, G. Reyne, F. Dumas-Bouchiat, Autonomous micro-magnet based systems for highly efficient magnetic separation, Applied Physics Letters, 99 (2011) 232504.

[36] N.H. Hai, N. Chau, N.H. Luong, N.T.V. Anh, P.T. Nghia, Applications of magnetite nanoparticles for water treatment and for DNA and cell separation, Journal of the Korean Physical Society, 53 (2008) 1601.

[37] J.-t. Jang, H. Nah, J.-H. Lee, S.H. Moon, M.G. Kim, J. Cheon, Critical Enhancements of MRI Contrast and Hyperthermic Effects by Dopant-Controlled Magnetic Nanoparticles, Angew. Chem., 121 (2009) 1260-1264.

[38] J.W.M. Bulte, D.L. Kraitchman, Iron oxide MR contrast agents for molecular and cellular imaging, NMR Biomed., 17 (2004) 484-499.

[39] A.L. Oldenburg, F.J.-J. Toublan, K.S. Suslick, A. Wei, S.A. Boppart, Magnetomotive contrast for in vivo optical coherence tomography, Optics Express, 13 (2005) 6597-6614.

[40] J. Barrick, A.L. Oldenburg, K.J. Lohmann, D.A. Ernst, Magnetomotive Optical Coherence Tomography as New Method for Endogenous Magnetite Detection, Biophysical Journal, 114 (2018) $176 a$.

[41] J. Li, B. Arnal, C.-W. Wei, J. Shang, T.-M. Nguyen, M. O'Donnell, X. Gao, Magneto-Optical Nanoparticles for Cyclic Magnetomotive Photoacoustic Imaging, ACS Nano, 9 (2015) 1964-1976.

[42] D.J. Grootendorst, J. Jose, R.M. Fratila, M. Visscher, A.H. Velders, B. Ten Haken, T.G. Van Leeuwen, W. Steenbergen, S. Manohar, T.J.M. Ruers, Evaluation of superparamagnetic iron oxide nanoparticles (Endorem ${ }^{\circledR}$ ) as a photoacoustic contrast agent for intra-operative nodal staging, Contrast media \& molecular imaging, 8 (2013) 83-91.

[43] B. Gleich, J. Weizenecker, Tomographic imaging using the nonlinear response of magnetic particles, Nature, 435 (2005) 1214-1217.

[44] O. Junghwan, D.F. Marc, K. Jeehyun, C. Chris, E. Stanislav, E.M. Thomas, Detection of magnetic nanoparticles in tissue using magneto-motive ultrasound, Nanotechnology, 17 (2006) 4183.

[45] R. John, S. A Boppart, Magnetomotive molecular nanoprobes, Curr. Med. Chem., 18 (2011) 21032114.

[46] T.W.J. Almeida, D.R.T. Sampaio, T.Z. Pavan, A.A.O. Carneiro, Shear wave Vibro Magneto Acoustography for measuring tissue mimicking phantom elasticity and viscosity, in: Ultrasonics Symposium (IUS), 2014 IEEE International, 2014, pp. 1097-1100.

[47] T.W.J. Almeida, D.R.T. Sampaio, A.C. Bruno, T.Z. Pavan, A.A.O. Carneiro, Comparison between shear wave dispersion magneto motive ultrasound and transient elastography for measuring tissuemimicking phantom viscoelasticity, IEEE Transactions on Ultrasonics, Ferroelectrics, and Frequency Control, 62 (2015) 2138-2145. 
[48] M. Mehrmohammadi, T.-H. Shin, M. Qu, P. Kruizinga, R.L. Truby, J.-H. Lee, J. Cheon, S.Y. Emelianov, In vivo pulsed magneto-motive ultrasound imaging using high-performance magnetoactive contrast nanoagents, Nanoscale, 5 (2013) 11179-11186.

[49] M.W. Wilson, R.K. Kerlan, N.A. Fidelman, A.P. Venook, J.M. LaBerge, J. Koda, R.L. Gordon, Hepatocellular Carcinoma: Regional Therapy with a Magnetic Targeted Carrier Bound to Doxorubicin in a Dual MR Imaging/ Conventional Angiography Suite-Initial Experience with Four Patients, Radiology, 230 (2004) 287-293.

[50] O. Veiseh, J.W. Gunn, M. Zhang, Design and fabrication of magnetic nanoparticles for targeted drug delivery and imaging, Advanced drug delivery reviews, 62 (2010) 284-304.

[51] N. Gao, E.N. Bozeman, W. Qian, L. Wang, H. Chen, M. Lipowska, C.A. Staley, Y.A. Wang, H. Mao, L. Yang, Tumor penetrating theranostic nanoparticles for enhancement of targeted and image-guided drug delivery into peritoneal tumors following intraperitoneal delivery, Theranostics, 7 (2017) 1689.

[52] T. Chen, M.I. Shukoor, R. Wang, Z. Zhao, Q. Yuan, S. Bamrungsap, X. Xiong, W. Tan, Smart Multifunctional Nanostructure for Targeted Cancer Chemotherapy and Magnetic Resonance Imaging, ACS Nano, 5 (2011) 7866-7873.

[53] T.-Y. Liu, K.-H. Liu, D.-M. Liu, S.-Y. Chen, I.W. Chen, Temperature-Sensitive Nanocapsules for Controlled Drug Release Caused by Magnetically Triggered Structural Disruption, Advanced Functional Materials, 19 (2009) 616-623.

[54] B. Thiesen, A. Jordan, Clinical applications of magnetic nanoparticles for hyperthermia, International Journal of Hyperthermia, 24 (2008) 467-474.

[55] R. Gilchrist, R. Medal, W.D. Shorey, R.C. Hanselman, J.C. Parrott, C.B. Taylor, Selective inductive heating of lymph nodes, Annals of surgery, 146 (1957) 596.

[56] P. Southern, Q.A. Pankhurst, Commentary on the clinical and preclinical dosage limits of interstitially administered magnetic fluids for therapeutic hyperthermia based on current practice and efficacy models, International Journal of Hyperthermia, 34 (2018) 671-686.

[57] E.A. Périgo, G. Hemery, O. Sandre, D. Ortega, E. Garaio, F. Plazaola, F.J. Teran, Fundamentals and advances in magnetic hyperthermia, Applied Physics Reviews, 2 (2015) 041302.

[58] K. Mahmoudi, A. Bouras, D. Bozec, R. Ivkov, C. Hadjipanayis, Magnetic hyperthermia therapy for the treatment of glioblastoma: a review of the therapy's history, efficacy and application in humans, International Journal of Hyperthermia, 34 (2018) 1316-1328.

[59] S. Laurent, D. Forge, M. Port, A. Roch, C. Robic, L. Vander Elst, R.N. Muller, Magnetic Iron Oxide Nanoparticles: Synthesis, Stabilization, Vectorization, Physicochemical Characterizations, and Biological Applications, Chem. Rev., 108 (2008) 2064-2110.

[60] Q.A. Pankhurst, N.T.K. Thanh, S.K. Jones, J. Dobson, Progress in applications of magnetic nanoparticles in biomedicine, Journal of Physics D: Applied Physics, 42 (2009) 224001.

[61] S. He, H. Zhang, Y. Liu, F. Sun, X. Yu, X. Li, L. Zhang, L. Wang, K. Mao, G. Wang, Y. Lin, Z. Han, R. Sabirianov, H. Zeng, Magnetic Nanoparticle Hyperthermia: Maximizing Specific Loss Power for Magnetic Hyperthermia by Hard-Soft Mixed Ferrites (Small 29/2018), Small, 14 (2018) 1870133.

[62] Q.-s. Tang, D.-s. Zhang, X.-m. Cong, M.-I. Wan, L.-q. Jin, Using thermal energy produced by irradiation of $\mathrm{Mn}-\mathrm{Zn}$ ferrite magnetic nanoparticles (MZF-NPs) for heat-inducible gene expression, Biomaterials, 29 (2008) 2673-2679.

[63] M. Jeun, S.J. Moon, H. Kobayashi, H.Y. Shin, A. Tomitaka, Y.J. Kim, Y. Takemura, S.H. Paek, K.H. Park, K.-W. Chung, S. Bae, Effects of Mn concentration on the ac magnetically induced heating characteristics of superparamagnetic MnxZn1-xFe2O4 nanoparticles for hyperthermia., Applied Physics Letters, 96 (2010) 202511.

[64] J. Xie, C. Yan, Y. Yan, L. Chen, L. Song, F. Zang, Y. An, G. Teng, N. Gu, Y. Zhang, Multi-modal MnZn ferrite nanocrystals for magnetically-induced cancer targeted hyperthermia: a comparison of passive and active targeting effects, Nanoscale, 8 (2016) 16902-16915.

[65] M. Jeun, S. Park, G.H. Jang, K.H. Lee, Tailoring MgxMn1-xFe2O4 Superparamagnetic Nanoferrites for Magnetic Fluid Hyperthermia Applications, ACS Applied Materials \& Interfaces, 6 (2014) 1648716492. 
[66] Z. Beji, A. Hanini, L.S. Smiri, J. Gavard, K. Kacem, F. Villain, J.M. Grenèche, F. Chau, S. Ammar, Magnetic properties of $\mathrm{Zn}$-substituted $\mathrm{MnFe} 2 \mathrm{O} 4$ nanoparticles synthesized in polyol as potential heating agents for hyperthermia. Evaluation of their toxicity on Endothelial cells, Chemistry of Materials, 22 (2010) 5420-5429.

[67] S. Warner, Diagnostics + therapy = theranostics, in: The Scientist, 2004, pp. 38+.

[68] B. Sumer, J. Gao, Theranostic nanomedicine for cancer, Nanomedicine, 3 (2008) 137-140.

[69] A. Latorre, C. Posch, Y. Garcimartín, A. Celli, M. Sanlorenzo, I. Vujic, J. Ma, M. Zekhtser, K. Rappersberger, S. Ortiz-Urda, Á. Somoza, DNA and aptamer stabilized gold nanoparticles for targeted delivery of anticancer therapeutics, Nanoscale, 6 (2014) 7436-7442.

[70] L. Sun, D.Y. Joh, A. Al-Zaki, M. Stangl, S. Murty, J.J. Davis, B.C. Baumann, M. Alonso-Basanta, G.D. Kao, A. Tsourkas, Theranostic Application of Mixed Gold and Superparamagnetic Iron Oxide Nanoparticle Micelles in Glioblastoma Multiforme, Journal of Biomedical Nanotechnology, 12 (2016) 347-356.

[71] M. Ma, H. Chen, Y. Chen, X. Wang, F. Chen, X. Cui, J. Shi, Au capped magnetic core/mesoporous silica shell nanoparticles for combined photothermo-/chemo-therapy and multimodal imaging, Biomaterials, 33 (2012) 989-998.

[72] B. Sahoo, K.S.P. Devi, S. Dutta, T.K. Maiti, P. Pramanik, D. Dhara, Biocompatible mesoporous silicacoated superparamagnetic manganese ferrite nanoparticles for targeted drug delivery and MR imaging applications, Journal of colloid and interface science, 431 (2014) 31-41.

[73] Q. Liu, C. Jin, Y. Wang, X. Fang, X. Zhang, Z. Chen, W. Tan, Aptamer-conjugated nanomaterials for specific cancer cell recognition and targeted cancer therapy, Npg Asia Materials, 6 (2014) e95.

[74] C. Zhang, X. Ji, Y. Zhang, G. Zhou, X. Ke, H. Wang, P. Tinnefeld, Z. He, One-Pot Synthesized Aptamer-Functionalized CdTe:Zn2+ Quantum Dots for Tumor-Targeted Fluorescence Imaging in Vitro and in Vivo, Analytical Chemistry, 85 (2013) 5843-5849.

[75] S.A. Khan, R. Kanchanapally, Z. Fan, L. Beqa, A.K. Singh, D. Senapati, P.C. Ray, A gold nanocageCNT hybrid for targeted imaging and photothermal destruction of cancer cells, Chemical communications, 48 (2012) 6711-6713.

[76] J.-H. Lee, K. Lee, S.H. Moon, Y. Lee, T.G. Park, J. Cheon, All-in-One Target-Cell-Specific Magnetic Nanoparticles for Simultaneous Molecular Imaging and siRNA Delivery, Angewandte Chemie International Edition, 48 (2009) 4174-4179.

[77] H. Daniel, T. Zhi Wei, D. Rohan, Z. Bo, G. Patrick, R. Carlos, C. Steven, Combining magnetic particle imaging and magnetic fluid hyperthermia in a theranostic platform, Physics in Medicine \& Biology, 62 (2017) 3483.

[78] Z.W. Tay, P. Chandrasekharan, A. Chiu-Lam, D.W. Hensley, R. Dhavalikar, X.Y. Zhou, E.Y. Yu, P.W. Goodwill, B. Zheng, C. Rinaldi, S.M. Conolly, Magnetic Particle Imaging-Guided Heating in Vivo Using Gradient Fields for Arbitrary Localization of Magnetic Hyperthermia Therapy, ACS Nano, 12 (2018) 3699-3713.

[79] S. Liu, X. Feng, H. Jin, R. Zhang, Y. Luo, Z. Zheng, F. Gao, Y. Zhenga, Hand-held photoacoustic imager for theranostics in 3D, IEEE Trans. Med. Imaging, (2019) 1-1.

[80] W. Wu, Q. He, C. Jiang, Magnetic iron oxide nanoparticles: synthesis and surface functionalization strategies, Nanoscale research letters, 3 (2008) 397-415.

[81] Y.S. Kang, S. Risbud, J.F. Rabolt, P. Stroeve, Synthesis and Characterization of Nanometer-Size Fe3O4 and $y$-Fe2O3 Particles, Chemistry of Materials, 8 (1996) 2209-2211.

[82] J.-P. Fortin, C. Wilhelm, J. Servais, C. Ménager, J.-C. Bacri, F. Gazeau, Size-Sorted Anionic Iron Oxide Nanomagnets as Colloidal Mediators for Magnetic Hyperthermia, Journal of the American Chemical Society, 129 (2007) 2628-2635.

[83] R. Di Corato, G. Béalle, J. Kolosnjaj-Tabi, A. Espinosa, O. Clément, A.K.A. Silva, C. Ménager, C. Wilhelm, Combining Magnetic Hyperthermia and Photodynamic Therapy for Tumor Ablation with Photoresponsive Magnetic Liposomes, ACS Nano, 9 (2015) 2904-2916.

[84] D.S. Mathew, R.-S. Juang, An overview of the structure and magnetism of spinel ferrite nanoparticles and their synthesis in microemulsions, Chemical Engineering Journal, 129 (2007) 51-65. 
[85] M. Faraji, Y. Yamini, M. Rezaee, Magnetic nanoparticles: synthesis, stabilization, functionalization, characterization, and applications, Journal of the Iranian Chemical Society, 7 (2010) 1-37.

[86] X. Peng, H. Chen, J. Huang, H. Mao, D.M. Shin, Targeted magnetic iron oxide nanoparticles for tumor imaging and therapy, in: Biomedical Engineering-From Theory to Applications, InTech, 2011.

[87] S. Majidi, F. Zeinali Sehrig, S.M. Farkhani, M. Soleymani Goloujeh, A. Akbarzadeh, Current methods for synthesis of magnetic nanoparticles, Artificial Cells, Nanomedicine, and Biotechnology, 44 (2016) 722-734.

[88] S. Sun, H. Zeng, D.B. Robinson, S. Raoux, P.M. Rice, S.X. Wang, G. Li, Monodisperse MFe2O4 (M = $\mathrm{Fe}, \mathrm{Co}, \mathrm{Mn}$ ) Nanoparticles, Journal of the American Chemical Society, 126 (2004) 273-279.

[89] Y. Piñeiro, Z. Vargas, J. Rivas, M.A. López-Quintela, Iron Oxide Based Nanoparticles for Magnetic Hyperthermia Strategies in Biological Applications, Eur. J. Inorg. Chem., 2015 (2015) 4495-4509.

[90] Y. Hadadian, D.R.T. Sampaio, A.P. Ramos, A.A.O. Carneiro, M. Mozaffari, L.C. Cabrelli, T.Z. Pavan, Synthesis and characterization of zinc substituted magnetite nanoparticles and their application to magneto-motive ultrasound imaging, Journal of Magnetism and Magnetic Materials, 465 (2018) 3343.

[91] Y. Hadadian, A.P. Ramos, D. Sampaio, A. Carneiro, T. Pavan, Magneto-motive ultrasound imaging using superparamagnetic ferrite nanoparticles with enhanced saturation magnetization synthesized by a simple copreciptation method, in: 2017 IEEE International Ultrasonics Symposium (IUS), 2017, pp. 1-4.

[92] Y. Hadadian, M. Azimbagirad, E.A. Navas, T.Z. Pavan, A versatile induction heating system for magnetic hyperthermia studies under different experimental conditions, Review of Scientific Instruments, 90 (2019) 074701.

[93] Y. Hadadian, A.P. Ramos, T.Z. Pavan, Role of zinc substitution in magnetic hyperthermia properties of magnetite nanoparticles: interplay between intrinsic properties and dipolar interactions, Scientific Reports, 9 (2019) 18048.

[94] Y. Hadadian, J.H. Uliana, A.A.O. Carneiro, T.Z. Pavan, A Novel Theranostic Platform: Integration of Magnetomotive and Thermal Ultrasound Imaging with Magnetic Hyperthermia, IEEE Transactions on Biomedical Engineering, (2020) 1-1, DOI: 10.1109/TBME.2020.2990873.

[95] N. Buske, Application of magnetite sols in environmental technology, in: M.J. Schwuger, F.H. Haegel (Eds.) Surfactants and Colloids in the Environment, Steinkopff, 1994, pp. 175-180.

[96] J.I. Taylor, C.D. Hurst, M.J. Davies, N. Sachsinger, I.J. Bruce, Application of magnetite and silicamagnetite composites to the isolation of genomic DNA, Journal of Chromatography A, 890 (2000) 159166.

[97] J. Llandro, J. Palfreyman, A. Ionescu, C. Barnes, Magnetic biosensor technologies for medical applications: a review, Med Biol Eng Comput, 48 (2010) 977-998.

[98] G.A. Mansoori, P. Mohazzabi, P. McCormack, S. Jabbari, Nanotechnology in cancer prevention, detection and treatment: bright future lies ahead, World Review of Science, Technology and Sustainable Development, 4 (2007) 226-257.

[99] M. Johannsen, U. Gneveckow, L. Eckelt, A. Feussner, N. WaldÖFner, R. Scholz, S. Deger, P. Wust, S.A. Loening, A. Jordan, Clinical hyperthermia of prostate cancer using magnetic nanoparticles: Presentation of a new interstitial technique, International Journal of Hyperthermia, 21 (2005) 637647.

[100] D. Carta, M.F. Casula, A. Falqui, D. Loche, G. Mountjoy, C. Sangregorio, A. Corrias, A Structural and Magnetic Investigation of the Inversion Degree in Ferrite Nanocrystals MFe2O4 (M = Mn, Co, Ni), The Journal of Physical Chemistry C, 113 (2009) 8606-8615.

[101] A.R. West, Solid State Chemistry and its Applications, John Wiley \& Sons:Singapore

1989.

[102] M. Mozaffari, Y. Hadadian, A. Aftabi, M. Oveisy Moakhar, The effect of cobalt substitution on magnetic hardening of magnetite, Journal of Magnetism and Magnetic Materials, 354 (2014) 119-124. 
[103] D. Stamopoulos, E. Manios, V. Gogola, D. Niarchos, M. Pissas, On the Biocompatibility of Fe3O4 Ferromagnetic Nanoparticles with Human Blood Cells, Journal of Nanoscience and Nanotechnology, 10 (2010) 6110-6115.

[104] P. Tartaj, M.P. Morales, T. Gonzalez-Carreño, S. Veintemillas-Verdaguer, C.J. Serna, The Iron Oxides Strike Back: From Biomedical Applications to Energy Storage Devices and Photoelectrochemical Water Splitting, Advanced Materials, 23 (2011) 5243-5249.

[105] M. Mascolo, Y. Pei, T. Ring, Room Temperature Co-Precipitation Synthesis of Magnetite Nanoparticles in a Large pH Window with Different Bases, Materials, 6 (2013) 5549.

[106] A. Aliakbari, M. Seifi, S. Mirzaee, H. Hekmatara, Influence of different synthesis conditions on properties of oleic acid-coated-Fe3O4 nanoparticles, Materials Science-Poland, 33 (2015) 100-106.

[107] H. Hejase, S.S. Hayek, S. Qadri, Y. Haik, MnZnFe nanoparticles for self-controlled magnetic hyperthermia, Journal of Magnetism and Magnetic Materials, 324 (2012) 3620-3628.

[108] S. Karen, Thermosensitive magnetic nanoparticles for self-controlled hyperthermia cancer treatment, Journal of Nanomedicine \& Nanotechnology, 3 (2012) 1.

[109] A. Bumb, M.W. Brechbiel, P.L. Choyke, L. Fugger, A. Eggeman, D. Prabhakaran, J. Hutchinson, P.J. Dobson, Synthesis and characterization of ultra-small superparamagnetic iron oxide nanoparticles thinly coated with silica, Nanotechnology, 19 (2008) 335601.

[110] B. Jeyadevan, K. Tohji, K. Nakatsuka, Structure analysis of coprecipitated ZnFe2O4 by extended x-ray-absorption fine structure, Journal of Applied Physics, 76 (1994) 6325-6327.

[111] I.H. Gul, W. Ahmed, A. Maqsood, Electrical and magnetic characterization of nanocrystalline Ni$\mathrm{Zn}$ ferrite synthesis by co-precipitation route, Journal of Magnetism and Magnetic Materials, 320 (2008) $270-275$.

[112] S.S. Jadhav, S.E. Shirsath, S.M. Patange, K.M. Jadhav, Effect of Zn substitution on magnetic properties of nanocrystalline cobalt ferrite, Journal of Applied Physics, 108 (2010) 093920.

[113] R. Valenzuela, Magnetic Ceramics, Cambridge University Press, 1994.

[114] A. Navrotsky, O.J. Kleppa, Thermodynamics of formation of simple spinels, Journal of Inorganic and Nuclear Chemistry, 30 (1968) 479-498.

[115] C. Upadhyay, H.C. Verma, S. Anand, Cation distribution in nanosized Ni-Zn ferrites, Journal of Applied Physics, 95 (2004) 5746-5751.

[116] H. Arend, J. Hulliger, N.A.T.O.S.A. Division, Crystal Growth in Science and Technology, Plenum Press, 1989.

[117] D.V. Kurmude, R.S. Barkule, A.V. Raut, D.R. Shengule, K.M. Jadhav, X-Ray Diffraction and Cation Distribution Studies in Zinc-Substituted Nickel Ferrite Nanoparticles, Journal of Superconductivity and Novel Magnetism, 27 (2014) 547-553.

[118] B.D. Cullity, S.R. Stock, Elements of X-Ray Diffraction: Pearson New International Edition, Pearson Education Limited, 2013.

[119] S.S. JADHAV, S.E. SHIRSATH, B.G. TOKSHA, S.M. PATANGE, S.J. SHUKLA, K.M. JADHAV, STRUCTURAL PROPERTIES AND CATION DISTRIBUTION OF CO-Zn NANOFERRITES, International Journal of Modern Physics B, 23 (2009) 5629-5638.

[120] J.M. Byrne, V.S. Coker, E. Cespedes, P.L. Wincott, D.J. Vaughan, R.A.D. Pattrick, G. van der Laan, E. Arenholz, F. Tuna, M. Bencsik, J.R. Lloyd, N.D. Telling, Biosynthesis of Zinc Substituted Magnetite Nanoparticles with Enhanced Magnetic Properties, Advanced Functional Materials, 24 (2014) 25182529.

[121] P.M. Zélis, G.A. Pasquevich, S.J. Stewart, M.B.F.v. Raap, J. Aphesteguy, I.J. Bruvera, C. Laborde, B. Pianciola, S. Jacobo, F.H. Sánchez, Structural and magnetic study of zinc-doped magnetite nanoparticles and ferrofluids for hyperthermia applications, Journal of Physics D: Applied Physics, 46 (2013) 125006.

[122] M.G. Brik, A. Suchocki, A. Kamińska, Lattice Parameters and Stability of the Spinel Compounds in Relation to the Ionic Radii and Electronegativities of Constituting Chemical Elements, Inorganic Chemistry, 53 (2014) 5088-5099. 
[123] R. Shannon, Revised effective ionic radii and systematic studies of interatomic distances in halides and chalcogenides, AcCrA, 32 (1976) 751-767.

[124] I. Vedernikova, SYNTHESIS AND EVALUATION OF ZINC SUBSTITUTED MAGNETITE NANOPARTICLES FOR DRUG DELIVERY SYSTEMS, 2015, (2015) 4.

[125] M. Mozaffari, S. Shatooti, M. Jafarzadeh, M. Niyaifar, A. Aftabi, H. Mohammadpour, S. Amiri, Synthesis of $\mathrm{Zn} 2+$ substituted maghemite nanoparticles and investigation of their structural and magnetic properties, Journal of Magnetism and Magnetic Materials, 382 (2015) 366-375.

[126] W. Kim, C.-Y. Suh, S.-W. Cho, K.-M. Roh, H. Kwon, K. Song, I.-J. Shon, A new method for the identification and quantification of magnetite-maghemite mixture using conventional $\mathrm{X}$-ray diffraction technique, Talanta, 94 (2012) 348-352.

[127] A. Bocanegra-Diaz, N.D.S. Mohallem, R.D. Sinisterra, Preparation of a ferrofluid using cyclodextrin and magnetite, Journal of the Brazilian Chemical Society, 14 (2003) 936-941.

[128] M.M. Can, S. Ozcan, A. Ceylan, T. Firat, Effect of milling time on the synthesis of magnetite nanoparticles by wet milling, Materials Science and Engineering: B, 172 (2010) 72-75.

[129] I. Nedkov, T. Merodiiska, L. Slavov, R.E. Vandenberghe, Y. Kusano, J. Takada, Surface oxidation, size and shape of nano-sized magnetite obtained by co-precipitation, Journal of Magnetism and Magnetic Materials, 300 (2006) 358-367.

[130] L. Neel, Magnetic properties of ferrites: ferrimagnetism and antiferromagnetism, Ann. Phys, 3 (1948) 137-198.

[131] C. Venkataraju, G. Sathishkumar, K. Sivakumar, Effect of cation distribution on the structural and magnetic properties of nickel substituted nanosized $\mathrm{Mn}-\mathrm{Zn}$ ferrites prepared by co-precipitation method, Journal of Magnetism and Magnetic Materials, 322 (2010) 230-233.

[132] G.F. Goya, T.S. Berquó, F.C. Fonseca, M.P. Morales, Static and dynamic magnetic properties of spherical magnetite nanoparticles, Journal of Applied Physics, 94 (2003) 3520-3528.

[133] A.K. Gupta, M. Gupta, Synthesis and surface engineering of iron oxide nanoparticles for biomedical applications, Biomaterials, 26 (2005) 3995-4021.

[134] S. Sun, H. Zeng, Size-Controlled Synthesis of Magnetite Nanoparticles, Journal of the American Chemical Society, 124 (2002) 8204-8205.

[135] J.M.D. Coey, Noncollinear Spin Arrangement in Ultrafine Ferrimagnetic Crystallites, Physical Review Letters, 27 (1971) 1140-1142.

[136] J.M.D. Coey, Noncollinear spin structures, Canadian Journal of Physics, 65 (1987) 1210-1232.

[137] G. Muscas, G. Concas, C. Cannas, A. Musinu, A. Ardu, F. Orrù, D. Fiorani, S. Laureti, D. Rinaldi, G. Piccaluga, D. Peddis, Magnetic Properties of Small Magnetite Nanocrystals, The Journal of Physical Chemistry C, 117 (2013) 23378-23384.

[138] P.B. Shete, R.M. Patil, B.M. Tiwale, S.H. Pawar, Water dispersible oleic acid-coated Fe304 nanoparticles for biomedical applications, Journal of Magnetism and Magnetic Materials, 377 (2015) 406-410.

[139] Q.A. Pankhurst, R.J. Pollard, Origin of the spin-canting anomaly in small ferrimagnetic particles, Physical Review Letters, 67 (1991) 248-250.

[140] R.A.D. PATTRICK, G. VAN DER LAAN, C.M.B. HENDERSON, P. KUIPER, E. DUDZIK, D.J. VAUGHAN, Cation site occupancy in spinel ferrites studied by $\mathrm{X}$-ray magnetic circular dichroism, Eur. J. Mineral., 14 (2002) 1095-1102.

[141] E.J.W. Verwey, E.L. Heilmann, Physical Properties and Cation Arrangement of Oxides with Spinel Structures I. Cation Arrangement in Spinels, The Journal of Chemical Physics, 15 (1947) 174-180.

[142] E. Petrova, D. Kotsikau, V. Pankov, Structural characterization and magnetic properties of solgel derived ZnxFe3-xO4 nanoparticles, Journal of Magnetism and Magnetic Materials, 378 (2015) 429435.

[143] L.W. Yeary, J.-W. Moon, C.J. Rawn, L.J. Love, A.J. Rondinone, J.R. Thompson, B.C. Chakoumakos, T.J. Phelps, Magnetic properties of bio-synthesized zinc ferrite nanoparticles, Journal of Magnetism and Magnetic Materials, 323 (2011) 3043-3048. 
[144] P. Kinnari, R.V. Upadhyay, R.V. Mehta, Magnetic properties of Fe-Zn ferrite substituted ferrofluids, Journal of Magnetism and Magnetic Materials, 252 (2002) 35-38.

[145] Q. Tian, Q. Wang, Q. Xie, J. Li, Aqueous Solution Preparation, Structure, and Magnetic Properties of Nano-Granular Zn x Fe 3- x O 4 Ferrite Films, Nanoscale Res Lett, 5 (2010) 1518.

[146] E. Rezlescu, L. Sachelarie, P.D. Popa, N. Rezlescu, Effect of substitution of divalent ions on the electrical and magnetic properties of Ni-Zn-Me ferrites, Magnetics, IEEE Transactions on, 36 (2000) 3962-3967.

[147] E.C. Stoner, E.P. Wohlfarth, A Mechanism of Magnetic Hysteresis in Heterogeneous Alloys, Philosophical Transactions of the Royal Society of London. Series A, Mathematical and Physical Sciences, 240 (1948) 599-642.

[148] C.S. Grommé, T.L. Wright, D.L. Peck, Magnetic properties and oxidation of iron-titanium oxide minerals in Alae and Makaopuhi Lava Lakes, Hawaii, Journal of Geophysical Research, 74 (1969) 52775293.

[149] A.S. Hudson, Molecular engineering in the design of microwave ferrimagnetic garnets, RvPT, 1 (1970) 9.

[150] C.M. Srivastava, S.N. Shringi, R.G. Srivastava, N.G. Nanadikar, Magnetic ordering and domainwall relaxation in zinc-ferrous ferrites, Physical Review B, 14 (1976) 2032-2040.

[151] J. Takaobushi, H. Tanaka, T. Kawai, S. Ueda, J.-J. Kim, M. Kobata, E. Ikenaga, M. Yabashi, K. Kobayashi, Y. Nishino, D. Miwa, K. Tamasaku, T. Ishikawa, Fe3-xZnxO4 thin film as tunable high Curie temperature ferromagnetic semiconductor, Applied Physics Letters, 89 (2006) 242507.

[152] I. Sharifi, H. Shokrollahi, S. Amiri, Ferrite-based magnetic nanofluids used in hyperthermia applications, Journal of Magnetism and Magnetic Materials, 324 (2012) 903-915.

[153] M. A, K. C, Low Curie-transition temperature and superparamagnetic nature of $\mathrm{Fe} 304$ nanoparticles prepared by colloidal nanocrystal synthesis, Materials Chemistry and Physics, 192 (2017) 235-243.

[154] D. Thapa, V.R. Palkar, M.B. Kurup, S.K. Malik, Properties of magnetite nanoparticles synthesized through a novel chemical route, Materials Letters, 58 (2004) 2692-2694.

[155] B. Kalska-Szostko, U. Wykowska, D. Satula, P. Nordblad, Thermal treatment of magnetite nanoparticles, Beilstein Journal of Nanotechnology, 6 (2015) 1385-1396.

[156] L.A. Welo, O. Baudisch, XXXIX. The two-staye transformation of magnetite into hematite, Philosophical Magazine Series 6, 50 (1925) 399-408.

[157] C. Rath, S. Anand, R.P. Das, K.K. Sahu, S.D. Kulkarni, S.K. Date, N.C. Mishra, Dependence on cation distribution of particle size, lattice parameter, and magnetic properties in nanosize $\mathrm{Mn}-\mathrm{Zn}$ ferrite, Journal of Applied Physics, 91 (2002) 2211-2215.

[158] W. Wolski, E. Wolska, J. Kaczmarek, P. Piszora, Formation of manganese ferrite by modified hydrothermal method, physica status solidi (a), 152 (1995) K19-K22.

[159] C. Rath, K.K. Sahu, S. Anand, S.K. Date, N.C. Mishra, R.P. Das, Preparation and characterization of nanosize Mn-Zn ferrite, Journal of Magnetism and Magnetic Materials, 202 (1999) 77-84.

[160] X. Huang, I.H. El-Sayed, W. Qian, M.A. El-Sayed, Cancer Cell Imaging and Photothermal Therapy in the Near-Infrared Region by Using Gold Nanorods, Journal of the American Chemical Society, 128 (2006) 2115-2120.

[161] A.L. Oldenburg, M.N. Hansen, D.A. Zweifel, A. Wei, S.A. Boppart, Plasmon-resonant gold nanorods as low backscattering albedo contrast agents, Optics Express, 14 (2006) 6724-6738.

[162] R. Weissleder, U. Mahmood, Molecular Imaging, Radiology, 219 (2001) 316-333.

[163] G. Brix, E.A. Nekolla, D. Nosske, J. Griebel, Risks and safety aspects related to PET/MR examinations, Eur. J. Nucl. Med. Mol. Imag., 36 (2009) 131-138.

[164] D.E. Sosnovik, R. Weissleder, Emerging concepts in molecular MRI, Curr. Opin. Biotechnol., 18 (2007) 4-10.

[165] M.A. Haidekker, Medical Imaging Technology, Springer New York, 2013.

[166] R.R. Edelman, S. Warach, Magnetic Resonance Imaging, New Engl. J. Med., 328 (1993) 785-791.

[167] P.N.T. Wells, Ultrasound imaging, Physics in Medicine and Biology, 51 (2006) R83-R98. 
[168] H. Azhari, Basics of Biomedical Ultrasound for Engineers, Wiley, 2010.

[169] K.K. Shung, Diagnostic Ultrasound: Imaging and Blood Flow Measurements, CRC Press, 2005.

[170] M. Evertsson, P. Kjellman, M. Cinthio, R. Andersson, T.A. Tran, G. Grafström, H. Toftevall, S. Fredriksson, C. Ingvar, S.-E. Strand, Combined Magnetomotive ultrasound, PET/CT, and MR imaging of $68 \mathrm{Ga}$-labelled superparamagnetic iron oxide nanoparticles in rat sentinel lymph nodes in vivo, Scientific Reports, 7 (2017) 4824.

[171] T.Z. Pavan, D.R.T. Sampaio, A.A.O. Carneiro, D.T. Covas, Ultrasound-based transient elastography using a magnetic excitation, in: Ultrasonics Symposium (IUS), 2012 IEEE International, 2012, pp. 1846-1849.

[172] R.M. Ferguson, K.R. Minard, K.M. Krishnan, Optimization of nanoparticle core size for magnetic particle imaging, Journal of Magnetism and Magnetic Materials, 321 (2009) 1548-1551.

[173] A.G. Próspero, C.C. Quini, A.F. Bakuzis, P. Fidelis-de-Oliveira, G.M. Moretto, F.P. Mello, M.F. Calabresi, R.V. Matos, E.A. Zandoná, N. Zufelato, Real-time in vivo monitoring of magnetic nanoparticles in the bloodstream by AC biosusceptometry, Journal of nanobiotechnology, 15 (2017) 22.

[174] A. Colello Bruno, T.Z. Pavan, O. Baffa, A.A. Oliveira Carneiro, A hybrid transducer to magnetically and ultrasonically evaluate magnetic fluids, Ultrasonics, Ferroelectrics, and Frequency Control, IEEE Transactions on, 60 (2013) 2004-2012.

[175] A.C. Bruno, D.R.T. Sampaio, T.Z. Pavan, O. Baffa, A.A.O. Carneiro, A hybrid transducer to evaluate stomach emptying by ultrasound and susceptometric measurements: an in vivo feasibility study, IEEE Transactions on Ultrasonics, Ferroelectrics, and Frequency Control, 62 (2015) 1288-1294.

[176] J.G. Webster, M. Zborowski, J.J. Chalmers, Magnetophoresis: Fundamentals and Applications, in: Wiley Encyclopedia of Electrical and Electronics Engineering, 2015.

[177] M. Mehrmohammadi, J. Oh, L. Ma, E. Yantsen, T. Larson, S. Mallidi, S. Park, K.P. Johnston, K. Sokolov, T. Miner, S. Emelianov, Imaging of Iron Oxide Nanoparticles Using Magneto-Motive Ultrasound, in: Ultrasonics Symposium, 2007. IEEE, 2007, pp. 652-655.

[178] I.G. Lim, S. Park, J. Oh, Theoretical development of a magnetic force and an induced motion in elastic media for a magneto-motive technique, Journal of the Korean Physical Society, 69 (2016) 461471.

[179] R.Z. Azar, A. Baghani, S.E. Salcudean, R. Rohling, 2-D high-frame-rate dynamic elastography using delay compensated and angularly compounded motion vectors: Preliminary results, IEEE Transactions on Ultrasonics, Ferroelectrics, and Frequency Control, 57 (2010) 2421-2436.

[180] D.R.T. Sampaio, F.W. Grillo, A.C. Bruno, T.Z. Pavan, A.A.O. Carneiro, A magneto-motive ultrasound platform designed for pre-clinical and clinical applications, Research on Biomedical Engineering, 32 (2016) 337-346.

[181] J. Oudry, C. Bastard, V. Miette, R. Willinger, L. Sandrin, Copolymer-in-oil Phantom Materials for Elastography, Ultrasound Med. Biol., 35 (2009) 1185-1197.

[182] L. Cabrelli, C. , P. Pelissari, I. B. G. B. , A. Deana, M. , A. Carneiro, A. O. , T. Pavan, Z. , Stable phantom materials for ultrasound and optical imaging, Physics in Medicine \& Biology, 62 (2017) 432.

[183] L.C. Cabrelli, F.W. Grillo, D.R.T. Sampaio, A.A.O. Carneiro, T.Z. Pavan, Acoustic and Elastic Properties of Glycerol in Oil-Based Gel Phantoms, Ultrasound Med. Biol., 43 (2017) 2086-2094.

[184] F.W. Grillo, L.C. Cabrelli, D.R.T. Sampaio, A.A.O. Carneiro, T.Z. Pavan, Glycerol in oil-based phantom with improved performance for photoacoustic imaging, in: 2017 IEEE International Ultrasonics Symposium (IUS), 2017, pp. 1-4.

[185] C. Pereira, A.M. Pereira, C. Fernandes, M. Rocha, R. Mendes, M.P. Fernández-García, A. Guedes, P.B. Tavares, J.-M. Grenèche, J.P. Araújo, C. Freire, Superparamagnetic MFe2O4 (M = Fe, Co, Mn) Nanoparticles: Tuning the Particle Size and Magnetic Properties through a Novel One-Step Coprecipitation Route, Chemistry of Materials, 24 (2012) 1496-1504.

[186] K.Y. Yoon, M. Mehrmohammadi, A. Borwankar, S.Y. Emelianov, K.P. Johnston, Synthesis of Iron Oxide Nanoclusters with Enhanced Magnetization and Their Applications in Pulsed Magneto-Motive Ultrasound Imaging, Nano, 10 (2015) 1550073. 
[187] T.J. Hall, M. Bilgen, M.F. Insana, T.A. Krouskop, Phantom materials for elastography, IEEE Transactions on Ultrasonics, Ferroelectrics, and Frequency Control, 44 (1997) 1355-1365.

[188] T.Z. Pavan, E.L. Madsen, G.R. Frank, A. Adilton O Carneiro, T.J. Hall, Nonlinear elastic behavior of phantom materials for elastography, Physics in Medicine and Biology, 55 (2010) 2679-2692.

[189] C. Streffer, R. Engelhardt, J.W. Hand, F. Kallinowski, M. Molls, E. Scherer, D. van Beuningen, P. Vaupel, Hyperthermia and the Therapy of Malignant Tumors, Springer Berlin Heidelberg, 1987.

[190] R.W.Y. Habash, R. Bansal, D. Krewski, H.T. Alhafid, Thermal Therapy, Part 2: Hyperthermia Techniques, Critical Reviews ${ }^{\mathrm{TM}}$ in Biomedical Engineering, 34 (2006) 491-542.

[191] A. Chicheł, J. Skowronek, M. Kubaszewska, M. Kanikowski, Hyperthermia - description of a method and a review of clinical applications, Reports of Practical Oncology \& Radiotherapy, 12 (2007) 267-275.

[192] U. Gneveckow, A. Jordan, R. Scholz, V. Brüß, N. Waldöfner, J. Ricke, A. Feussner, B. Hildebrandt, B. Rau, P. Wust, Description and characterization of the novel hyperthermia- and thermoablationsystem MFH ${ }^{\circledR} 300 \mathrm{~F}$ for clinical magnetic fluid hyperthermia, Medical Physics, 31 (2004) 1444-1451.

[193] C.L. Dennis, R. Ivkov, Physics of heat generation using magnetic nanoparticles for hyperthermia, International Journal of Hyperthermia, 29 (2013) 715-729.

[194] I. Andreu, E. Natividad, Accuracy of available methods for quantifying the heat power generation of nanoparticles for magnetic hyperthermia, International Journal of Hyperthermia, 29 (2013) 739751.

[195] L.M. Lacroix, J. Carrey, M. Respaud, A frequency-adjustable electromagnet for hyperthermia measurements on magnetic nanoparticles, Review of Scientific Instruments, 79 (2008) 093909.

[196] M.E. Cano, A. Barrera, J.C. Estrada, A. Hernandez, T. Cordova, An induction heater device for studies of magnetic hyperthermia and specific absorption ratio measurements, Review of Scientific Instruments, 82 (2011) 114904.

[197] E.E. Mazon, E. Villa-Martínez, A. Hernández-Sámano, T. Córdova-Fraga, J.J. Ibarra-Sánchez, H.A. Calleja, J.A. Leyva Cruz, A. Barrera, J.C. Estrada, J.A. Paz, L.H. Quintero, M.E. Cano, A high-resolution frequency variable experimental setup for studying ferrofluids used in magnetic hyperthermia, Review of Scientific Instruments, 88 (2017) 084705.

[198] E.E. Mazon, A.H. Sámano, H. Calleja, L.H. Quintero, J.A. Paz, M.E. Cano, A frequency tuner for resonant inverters suitable for magnetic hyperthermia applications, Measurement Science and Technology, 28 (2017) 095901.

[199] E. Garaio, J M. Collantes, F. Plazaola, J A. Garcia, I. Castellanos-Rubio, A multifrequency eletromagnetic applicator with an integrated AC magnetometer for magnetic hyperthermia experiments, Measurement Science and Technology, 25 (2014) 115702.

[200] D.E. Bordelon, R.C. Goldstein, V.S. Nemkov, A. Kumar, J.K. Jackowski, T.L. DeWeese, R. Ivkov, Modified Solenoid Coil That Efficiently Produces High Amplitude AC Magnetic Fields With Enhanced Uniformity for Biomedical Applications, Magnetics, IEEE Transactions on, 48 (2012) 47-52.

[201] P.D. Barba, F. Dughiero, E. Sieni, Magnetic Field Synthesis in the Design of Inductors for Magnetic Fluid Hyperthermia, IEEE Transactions on Magnetics, 46 (2010) 2931-2934.

[202] P. Stauffer, P. Sneed, H. Hashemi, T. Phillips, Practical induction heating coil designs for clinical hyperthermia with ferromagnetic implants, IEEE Transactions on Biomedical Engineering, 41 (1994) 17-28.

[203] M.D. Bellar, T.S. Wu, A. Tchamdjou, J. Mahdavi, M. Ehsani, A review of soft-switched DC-AC converters, ITIA, 34 (1998) 847-860.

[204] A. Namadmalan, J.S. Moghani, J. Milimonfare, A current-fed parallel resonant push-pull inverter with a new cascaded coil flux control for induction heating applications, Journal of Power Electronics, 11 (2011) 632-638.

[205] A.W. Green, Modelling a push-pull parallel resonant convertor using generalised state-space averaging, IEE Proceedings B - Electric Power Applications, 140 (1993) 350-356. 
[206] D.J. Thrimawithana, U.K. Madawala, Modelling and analysis of split-capacitor push-pull parallelresonant converter in normal mode, International Journal of Industrial Electronics and Drives, 1 (2009) 42-51.

[207] K.H. Liu, Higher-efficiency cold-cathode fluorescent lamp power supply, in, Google Patents, 1997.

[208] M. Bakula, P. Pelgrims, R. Puers, A Wireless Powering and Communication System for Implantable Devices Based on a Royer Oscillator with Radio and Near-field Communication Links, Procedia Engineering, 120 (2015) 306-309.

[209] R.M. Zin, C.F. Soon, M.Z.A. Sani, E.R. Rizon, K.S. Tee, M.K. Ahmad, N.N. Ahmad, W.M. Jubadi, N. Nayan, Zero voltage switching driver and flyback transformer for generation of atmospheric pressure plasma jet, AIP Conf. Proc., 1883 (2017) 020023.

[210] D.A. Hapidin, I. Saleh, M.M. Munir, Khairurrijal, Design and Development of a Seriesconfiguration Mazzilli Zero Voltage Switching Flyback Converter as a High-voltage Power Supply for Needleless Electrospinning, Procedia Engineering, 170 (2017) 509-515.

[211] J.M. Alonso, J. Garcia, A.J. Calleja, J. Ribas, J. Cardesin, Analysis, design, and experimentation of a high-voltage power supply for ozone generation based on current-fed parallel-resonant push-pull inverter, ITIA, 41 (2005) 1364-1372.

[212] M. Usman, Inductive weld of joints for optical fiber pipe, in, Mid Sweden University, Department of Electronics Design, 2017, pp. 108.

[213] R.L. Bright, G.H. Royer, Electrical inverter circuits, in, Google Patents, 1957.

[214] A.I. Pressman, K. Billings, T. Morey, Switching Power Supply Design, 3rd Ed, McGraw-Hill Education, 2007.

[215] S.L. McClusky, High Voltage Resonant Self-Tracking Current-Fed Converter, in: Electrical Engineering, Faculty of California Polytechnic State University, San Luis Obispo, 2010.

[216] J.D. Paolucci, Novel Current-Fed Boundary-Mode Parallel-Resonant Push-Pull Converter, in: Electrical Engineering, Faculty of California Polytechnic State University, San Luis Obispo, 2009.

[217] A. Namadmalan, J.S. Moghani, Single-phase current source induction heater with improved efficiency and package size, Journal of Power Electronics, 13 (2013) 322-328.

[218] T. Liang, R. Chen, J. Chen, Current-Fed Parallel-Resonant DC-AC Inverter for ColdCathodeFluorescent Lamps With Zero-Current Switching, ITPE, 23 (2008) 2206-2210.

[219] P. Horowitz, W. Hill, The Art of Electronics, Cambridge University Press, 2015.

[220] Coil32.software.version11, Coil32 software version 11, in, 2018.

[221] A. Yu, X. Zeng, D. Xiong, M. Tian, J. Li, An Improved Autonomous Current-Fed Push-Pull ParallelResonant Inverter for Inductive Power Transfer System, Energies, 11 (2018) 2653.

[222] M.M. Mansour, M.M. Mansour, A. Mehrotra, Analysis of MOS cross-coupled LC-tank oscillators using short-channel device equations, in: Asia and South Pacific Design Automation Conference IEEE Press, 2004, pp. 181-185.

[223] P. Gas, A. Miaskowski, Specifying the ferrofluid parameters important from the viewpoint of magnetic fluid hyperthermia, in: 2015 Selected Problems of Electrical Engineering and Electronics (WZEE), 2015, pp. 1-6.

[224] A. Skumiel, B. Leszczyński, M. Molcan, M. Timko, The comparison of magnetic circuits used in magnetic hyperthermia, Journal of Magnetism and Magnetic Materials, 420 (2016) 177-184.

[225] M.G. Stewart, W.H. Siew, L.C. Campbell, M.G. Stewart, W.H. Siew, Design of portable electric and magnetic field generators, Measurement Science and Technology, 11 (2000) 1596.

[226] R. Olaru, A. Arcire, C. Petrescu, M.-M. Mihai, Study of the magnetic force delivered by an actuator with nonlinear ferrofluid and permanent magnets, IEEJ Transactions on Electrical and Electronic Engineering, 12 (2017) 24-30.

[227] P. Gas, E. Kurgan, Cooling effects inside water-cooled inductors for magnetic fluid hyperthermia, in: 2017 Progress in Applied Electrical Engineering (PAEE), 2017, pp. 1-4. 
[228] B. Minnaert, N. Stevens, Evaluation of the vertical magnetic field generated by a spiral planar coil, in: 2015 Nordic Circuits and Systems Conference (NORCAS): NORCHIP \& International Symposium on System-on-Chip (SoC), 2015, pp. 1-3.

[229] M. Kallumadil, M. Tada, T. Nakagawa, M. Abe, P. Southern, Q.A. Pankhurst, Suitability of commercial colloids for magnetic hyperthermia, Journal of Magnetism and Magnetic Materials, 321 (2009) 1509-1513.

[230] Y. Hadadian, D.R.T. Sampaio, A.P. Ramos, A.A.O. Carneiro, T.Z. Pavan, Magneto-motive ultrasound imaging using superparamagnetic ferrite nanoparticle with enhanced saturation magnetization synthesized by a simple copreciptation method, in: 2017 IEEE International Ultrasonics Symposium (IUS), 2017, pp. 1-4.

[231] T.R. Oliveira, P.R. Stauffer, C.-T. Lee, C.D. Landon, W. Etienne, K.A. Ashcraft, K.L. McNerny, A. Mashal, J. Nouls, P.F. Maccarini, W.F. Beyer, B. Inman, M.W. Dewhirst, Magnetic fluid hyperthermia for bladder cancer: A preclinical dosimetry study, International Journal of Hyperthermia, 29 (2013) 835-844.

[232] V. Connord, B. Mehdaoui, R.P. Tan, J. Carrey, M. Respaud, An air-cooled Litz wire coil for measuring the high frequency hysteresis loops of magnetic samples-A useful setup for magnetic hyperthermia applications, Review of Scientific Instruments, 85 (2014) 093904.

[233] A. Taylor, Y. Krupskaya, K. Krämer, S. Füssel, R. Klingeler, B. Büchner, M.P. Wirth, Cisplatin-loaded carbon-encapsulated iron nanoparticles and their in vitro effects in magnetic fluid hyperthermia, Carbon, 48 (2010) 2327-2334.

[234] M. Hedayati, A. Attaluri, D. Bordelon, R. Goh, M. Armour, H. Zhou, C. Cornejo, M. Wabler, Y. Zhang, T. DeWeese, R. Ivkov, New iron-oxide particles for magnetic nanoparticle hyperthermia: an invitro and in-vivo pilot study, Proc SPIE, 8584 (2013) 858404.

[235] Z. Wu, Z. Zhuo, D. Cai, J.a. Wu, J. Wang, J. Tang, An induction heating device using planar coil with high amplitude alternating magnetic fields for magnetic hyperthermia, Technol. Health Care, 23 (2015) S203-S209.

[236] M. Subramanian, A. Miaskowski, G. Pearce, J. Dobson, A coil system for real-time magnetic fluid hyperthermia microscopy studies, International Journal of Hyperthermia, 32 (2016) 112-120.

[237] D. Brizi, A. Monorchio, N. Fontana, A novel coil for highly focused magnetic hyperthermia with nanoparticles, in: 2017 IEEE International Symposium on Antennas and Propagation \& USNC/URSI National Radio Science Meeting, 2017, pp. 1223-1224.

[238] B. Razavi, RF Microelectronics, Prentice Hall, 2012.

[239] R.E. Rosensweig, Heating magnetic fluid with alternating magnetic field, Journal of Magnetism and Magnetic Materials, 252 (2002) 370-374.

[240] S. Ruta, R. Chantrell, O. Hovorka, Unified model of hyperthermia via hysteresis heating in systems of interacting magnetic nanoparticles, Scientific Reports, 5 (2015) 9090.

[241] M.S. Carrião, V.R.R. Aquino, G.T. Landi, E.L. Verde, M.H. Sousa, A.F. Bakuzis, Giant-spin nonlinear response theory of magnetic nanoparticle hyperthermia: A field dependence study, Journal of Applied Physics, 121 (2017) 173901.

[242] J. Carrey, B. Mehdaoui, M. Respaud, Simple models for dynamic hysteresis loop calculations of magnetic single-domain nanoparticles: Application to magnetic hyperthermia optimization, Journal of Applied Physics, 109 (2011) 083921.

[243] W.J. Atkinson, I.A. Brezovich, D.P. Chakraborty, Usable Frequencies in Hyperthermia with Thermal Seeds, IEEE Transactions on Biomedical Engineering, BME-31 (1984) 70-75.

[244] H. Rudolf, D. Silvio, M. Robert, Z. Matthias, Magnetic particle hyperthermia: nanoparticle magnetism and materials development for cancer therapy, Journal of Physics: Condensed Matter, 18 (2006) S2919.

[245] G.T. Landi, Role of dipolar interaction in magnetic hyperthermia, Physical Review B, 89 (2014) 014403.

[246] R.P. Tan, J. Carrey, M. Respaud, Magnetic hyperthermia properties of nanoparticles inside lysosomes using kinetic Monte Carlo simulations: Influence of key parameters and dipolar 
interactions, and evidence for strong spatial variation of heating power, Physical Review B, 90 (2014) 214421.

[247] B. Mehdaoui, R.P. Tan, A. Meffre, J. Carrey, S. Lachaize, B. Chaudret, M. Respaud, Increase of magnetic hyperthermia efficiency due to dipolar interactions in low-anisotropy magnetic nanoparticles: Theoretical and experimental results, Physical Review B, 87 (2013) 174419.

[248] C.L. Dennis, A.J. Jackson, J.A. Borchers, R. Ivkov, A.R. Foreman, J.W. Lau, E. Goernitz, C. Gruettner, The influence of collective behavior on the magnetic and heating properties of iron oxide nanoparticles, Journal of Applied Physics, 103 (2008) 07A319.

[249] D. Serantes, K. Simeonidis, M. Angelakeris, O. Chubykalo-Fesenko, M. Marciello, M.d.P. Morales, D. Baldomir, C. Martinez-Boubeta, Multiplying Magnetic Hyperthermia Response by Nanoparticle Assembling, The Journal of Physical Chemistry C, 118 (2014) 5927-5934.

[250] A.Y. Zubarev, Magnetic hyperthermia in a system of ferromagnetic particles, frozen in a carrier medium: Effect of interparticle interactions, PhRvE, 98 (2018) 032610.

[251] L.C. Branquinho, M.S. Carrião, A.S. Costa, N. Zufelato, M.H. Sousa, R. Miotto, R. Ivkov, A.F. Bakuzis, Effect of magnetic dipolar interactions on nanoparticle heating efficiency: Implications for cancer hyperthermia, Scientific Reports, 3 (2013) 2887.

[252] C. Blanco-Andujar, D. Ortega, P. Southern, Q.A. Pankhurst, N.T.K. Thanh, High performance multi-core iron oxide nanoparticles for magnetic hyperthermia: microwave synthesis, and the role of core-to-core interactions, Nanoscale, 7 (2015) 1768-1775.

[253] B. Mehdaoui, A. Meffre, J. Carrey, S. Lachaize, L.-M. Lacroix, M. Gougeon, B. Chaudret, M. Respaud, Optimal Size of Nanoparticles for Magnetic Hyperthermia: A Combined Theoretical and Experimental Study, Advanced Functional Materials, 21 (2011) 4573-4581.

[254] C. Haase, U. Nowak, Role of dipole-dipole interactions for hyperthermia heating of magnetic nanoparticle ensembles, Physical Review B, 85 (2012) 045435.

[255] C. Martinez-Boubeta, K. Simeonidis, D. Serantes, I. Conde-Leborán, I. Kazakis, G. Stefanou, L. Peña, R. Galceran, L. Balcells, C. Monty, D. Baldomir, M. Mitrakas, M. Angelakeris, Adjustable Hyperthermia Response of Self-Assembled Ferromagnetic Fe-MgO Core-Shell Nanoparticles by Tuning Dipole-Dipole Interactions, Advanced Functional Materials, 22 (2012) 3737-3744.

[256] R. Fu, Y. Yan, C. Roberts, Z. Liu, Y.J.S.r. Chen, The role of dipole interactions in hyperthermia heating colloidal clusters of densely-packed superparamagnetic nanoparticles, 8 (2018) 4704.

[257] P. de la Presa, Y. Luengo, M. Multigner, R. Costo, M.P. Morales, G. Rivero, A. Hernando, Study of Heating Efficiency as a Function of Concentration, Size, and Applied Field in $\gamma$-Fe2O3 Nanoparticles, The Journal of Physical Chemistry C, 116 (2012) 25602-25610.

[258] I. Andreu, E. Natividad, L. Solozábal, O. Roubeau, Nano-objects for Addressing the Control of Nanoparticle Arrangement and Performance in Magnetic Hyperthermia, ACS Nano, 9 (2015) 14081419.

[259] D. Niculaes, A. Lak, G.C. Anyfantis, S. Marras, O. Laslett, S.K. Avugadda, M. Cassani, D. Serantes, O. Hovorka, R. Chantrell, T. Pellegrino, Asymmetric Assembling of Iron Oxide Nanocubes for Improving Magnetic Hyperthermia Performance, ACS Nano, (2017).

[260] E. Myrovali, N. Maniotis, A. Makridis, A. Terzopoulou, V. Ntomprougkidis, K. Simeonidis, D. Sakellari, O. Kalogirou, T. Samaras, R. Salikhov, M. Spasova, M. Farle, U. Wiedwald, M. Angelakeris, Arrangement at the nanoscale: Effect on magnetic particle hyperthermia, Scientific Reports, 6 (2016) 37934.

[261] P. Guardia, R. Di Corato, L. Lartigue, C. Wilhelm, A. Espinosa, M. Garcia-Hernandez, F. Gazeau, L. Manna, T. Pellegrino, Water-Soluble Iron Oxide Nanocubes with High Values of Specific Absorption Rate for Cancer Cell Hyperthermia Treatment, ACS Nano, 6 (2012) 3080-3091.

[262] M. Albino, E. Fantechi, C. Innocenti, A. López-Ortega, V. Bonanni, G. Campo, F. Pineider, M. Gurioli, P. Arosio, T. Orlando, G. Bertoni, C. de Julián Fernández, A. Lascialfari, C. Sangregorio, Role of $\mathrm{Zn} 2+$ Substitution on the Magnetic, Hyperthermic, and Relaxometric Properties of Cobalt Ferrite Nanoparticles, The Journal of Physical Chemistry C, 123 (2019) 6148-6157. 
[263] Y. Yang, X. Liu, Y. Lv, T.S. Herng, X. Xu, W. Xia, T. Zhang, J. Fang, W. Xiao, J. Ding, Orientation Mediated Enhancement on Magnetic Hyperthermia of Fe3O4 Nanodisc, Advanced Functional Materials, 25 (2015) 812-820.

[264] A. Sathya, P. Guardia, R. Brescia, N. Silvestri, G. Pugliese, S. Nitti, L. Manna, T. Pellegrino, CoxFe3xO4 Nanocubes for Theranostic Applications: Effect of Cobalt Content and Particle Size, Chemistry of Materials, 28 (2016) 1769-1780.

[265] B. Behdadfar, A. Kermanpur, H. Sadeghi-Aliabadi, M.d.P. Morales, M. Mozaffari, Synthesis of

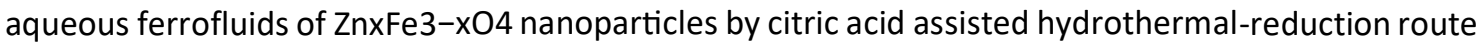
for magnetic hyperthermia applications, Journal of Magnetism and Magnetic Materials, 324 (2012) 2211-2217.

[266] V. Mameli, A. Musinu, A. Ardu, G. Ennas, D. Peddis, D. Niznansky, C. Sangregorio, C. Innocenti, N.T.K. Thanh, C. Cannas, Studying the effect of Zn-substitution on the magnetic and hyperthermic properties of cobalt ferrite nanoparticles, Nanoscale, 8 (2016) 10124-10137.

[267] H. Ghayour, M. Abdellahi, M.G. Nejad, A. Khandan, S. Saber-Samandari, Study of the effect of the $\mathrm{Zn} 2+$ content on the anisotropy and specific absorption rate of the cobalt ferrite: the application of Co1 - xZnxFe2O4 ferrite for magnetic hyperthermia, Journal of the Australian Ceramic Society, 54 (2018) 223-230.

[268] R.A. Bohara, N.D. Thorat, A.K. Chaurasia, S.H. Pawar, Cancer cell extinction through a magnetic fluid hyperthermia treatment produced by superparamagnetic Co-Zn ferrite nanoparticles, RSC Advances, 5 (2015) 47225-47234.

[269] A. Apostolov, I. Apostolova, J. Wesselinowa, Specific absorption rate in Zn-doted ferrites for selfcontrolled magnetic hyperthermia, The European Physical Journal B, 92 (2019) 58.

[270] A. Hanini, L. Lartigue, J. Gavard, K. Kacem, C. Wilhelm, F. Gazeau, F. Chau, S. Ammar, Zinc substituted ferrite nanoparticles with Zn0.9Fe2.104 formula used as heating agents for in vitro hyperthermia assay on glioma cells, Journal of Magnetism and Magnetic Materials, 416 (2016) 315320.

[271] R. Hergt, S. Dutz, Magnetic particle hyperthermia-biophysical limitations of a visionary tumour therapy, Journal of Magnetism and Magnetic Materials, 311 (2007) 187-192.

[272] F. Luis, J.M. Torres, L.M. García, J. Bartolomé, J. Stankiewicz, F. Petroff, F. Fettar, J.L. Maurice, A. Vaurès, Enhancement of the magnetic anisotropy of nanometer-sized Co clusters: Influence of the surface and of interparticle interactions, Physical Review B, 65 (2002) 094409.

[273] W.F. Brown, Theory of the Approach to Magnetic Saturation, Physical Review, 58 (1940) 736743.

[274] J.F. Herbst, F.E. Pinkerton, Law of approach to saturation for polycrystalline ferromagnets: Remanent initial state, Physical Review B, 57 (1998) 10733-10739.

[275] R.R. Wildeboer, P. Southern, Q.A. Pankhurst, On the reliable measurement of specific absorption rates and intrinsic loss parameters in magnetic hyperthermia materials, Journal of Physics D: Applied Physics, 47 (2014) 495003.

[276] N.R. Jana, C. Earhart, J.Y. Ying, Synthesis of Water-Soluble and Functionalized Nanoparticles by Silica Coating, Chemistry of Materials, 19 (2007) 5074-5082.

[277] D.J. Shaw, Introduction to colloid and surface chemistry, 4th ed., Butterworths, 1992.

[278] E. Lima, A.L. Brandl, A.D. Arelaro, G.F. Goya, Spin disorder and magnetic anisotropy in $\mathrm{Fe} 3 \mathrm{O} 4$ nanoparticles, Journal of Applied Physics, 99 (2006) 083908.

[279] C. Nayek, K. Manna, A.A. Imam, A.Y. Alqasrawi, I.M. Obaidat, Size-dependent magnetic anisotropy of PEG coated Fe 304 nanoparticles; comparing two magnetization methods, IOP Conference Series: Materials Science and Engineering, 305 (2018) 012012.

[280] A.A. McGhie, C. Marquina, K. O'Grady, G. Vallejo-Fernandez, Measurement of the distribution of anisotropy constants in magnetic nanoparticles for hyperthermia applications, Journal of Physics D: Applied Physics, 50 (2017) 455003. 
[281] M. Palihawadana-Arachchige, H. Nemala, V.M. Naik, R. Naik, Effect of magnetic dipolar interactions on temperature dependent magnetic hyperthermia in ferrofluids, Journal of Applied Physics, 121 (2017) 023901.

[282] J. Santoyo Salazar, L. Perez, O. de Abril, L. Truong Phuoc, D. Ihiawakrim, M. Vazquez, J.-M. Greneche, S. Begin-Colin, G. Pourroy, Magnetic Iron Oxide Nanoparticles in 10-40 nm Range: Composition in Terms of Magnetite/Maghemite Ratio and Effect on the Magnetic Properties, Chemistry of Materials, 23 (2011) 1379-1386.

[283] R. Frison, G. Cernuto, A. Cervellino, O. Zaharko, G.M. Colonna, A. Guagliardi, N. Masciocchi, Magnetite-Maghemite Nanoparticles in the 5-15 nm Range: Correlating the Core-Shell Composition and the Surface Structure to the Magnetic Properties. A Total Scattering Study, Chemistry of Materials, 25 (2013) 4820-4827.

[284] J. Park, K. An, Y. Hwang, J.-G. Park, H.-J. Noh, J.-Y. Kim, J.-H. Park, N.-M. Hwang, T. Hyeon, Ultralarge-scale syntheses of monodisperse nanocrystals, Nat Mater, 3 (2004) 891-895.

[285] N. Modaresi, R. Afzalzadeh, B. Aslibeiki, P. Kameli, A. Ghotbi Varzaneh, I. Orue, V.A. Chernenko, Magnetic properties of ZnxFe3-xO4 nanoparticles: A competition between the effects of size and $\mathrm{Zn}$ doping level, Journal of Magnetism and Magnetic Materials, 482 (2019) 206-218.

[286] M. Thakur, K. De, S. Giri, S. Si, A. Kotal, T.K. Mandal, Interparticle interaction and size effect in polymer coated magnetite nanoparticles, Journal of Physics: Condensed Matter, 18 (2006) 9093.

[287] F. B $\varnothing$ dker, S. Mørup, S. Linderoth, Surface effects in metallic iron nanoparticles, Physical Review Letters, 72 (1994) 282-285.

[288] F. Gazeau, J.C. Bacri, F. Gendron, R. Perzynski, Y.L. Raikher, V.I. Stepanov, E. Dubois, Magnetic resonance of ferrite nanoparticles:: evidence of surface effects, Journal of Magnetism and Magnetic Materials, 186 (1998) 175-187.

[289] H. Rudolf, D. Silvio, R. Michael, Effects of size distribution on hysteresis losses of magnetic nanoparticles for hyperthermia, Journal of Physics: Condensed Matter, 20 (2008) 385214.

[290] X.L. Liu, H.M. Fan, J.B. Yi, Y. Yang, E.S.G. Choo, J.M. Xue, D.D. Fan, J. Ding, Optimization of surface coating on $\mathrm{Fe} 3 \mathrm{O} 4$ nanoparticles for high performance magnetic hyperthermia agents, Journal of Materials Chemistry, 22 (2012) 8235-8244.

[291] R. Hergt, R. Hiergeist, M. Zeisberger, D. Schüler, U. Heyen, I. Hilger, W.A. Kaiser, Magnetic properties of bacterial magnetosomes as potential diagnostic and therapeutic tools, Journal of Magnetism and Magnetic Materials, 293 (2005) 80-86.

[292] S. Kossatz, R. Ludwig, H. Dähring, V. Ettelt, G. Rimkus, M. Marciello, G. Salas, V. Patel, F.J. Teran, I. Hilger, High Therapeutic Efficiency of Magnetic Hyperthermia in Xenograft Models Achieved with Moderate Temperature Dosages in the Tumor Area, Pharm. Res., 31 (2014) 3274-3288.

[293] P. Bender, J. Fock, C. Frandsen, M.F. Hansen, C. Balceris, F. Ludwig, O. Posth, E. Wetterskog, L.K. Bogart, P. Southern, W. Szczerba, L. Zeng, K. Witte, C. Grüttner, F. Westphal, D. Honecker, D. GonzálezAlonso, L. Fernández Barquín, C. Johansson, Relating Magnetic Properties and High Hyperthermia Performance of Iron Oxide Nanoflowers, The Journal of Physical Chemistry C, 122 (2018) 3068-3077. [294] L.A. Thomas, L. Dekker, M. Kallumadil, P. Southern, M. Wilson, S.P. Nair, Q.A. Pankhurst, I.P. Parkin, Carboxylic acid-stabilised iron oxide nanoparticles for use in magnetic hyperthermia, Journal of Materials Chemistry, 19 (2009) 6529-6535.

[295] S. Dutz, R. Hergt, Magnetic nanoparticle heating and heat transfer on a microscale: Basic principles, realities and physical limitations of hyperthermia for tumour therapy, International Journal of Hyperthermia, 29 (2013) 790-800.

[296] I. Conde-Leboran, D. Baldomir, C. Martinez-Boubeta, O. Chubykalo-Fesenko, M. del Puerto Morales, G. Salas, D. Cabrera, J. Camarero, F.J. Teran, D. Serantes, A Single Picture Explains Diversity of Hyperthermia Response of Magnetic Nanoparticles, The Journal of Physical Chemistry C, 119 (2015) 15698-15706.

[297] G. Diamantopoulos, G. Basina, V. Tzitzios, E. Karakosta, M. Fardis, Z. Jaglicic, N. Lazaridis, G. Papavassiliou, Magnetic hyperthermia of laponite based ferrofluid, Journal of Magnetism and Magnetic Materials, 336 (2013) 71-74. 
[298] N.A. Usov, Low frequency hysteresis loops of superparamagnetic nanoparticles with uniaxial anisotropy, Journal of Applied Physics, 107 (2010) 123909.

[299] A. Wang, J. Li, R. Gao, The structural force arising from magnetic interactions in polydisperse ferrofluids, Applied Physics Letters, 94 (2009) 212501.

[300] A.F. Abu-Bakr, A.Y. Zubarev, Hyperthermia in a system of interacting ferromagnetic particles under rotating magnetic field, Journal of Magnetism and Magnetic Materials, 477 (2019) 404-407.

[301] R.D.R. Kahmei, J.P. Borah, Clustering of MnFe 204 nanoparticles and the effect of field intensity in the generation of heat for hyperthermia application, Nanotechnology, 30 (2019) 035706.

[302] D. Serantes, D. Baldomir, C. Martinez-Boubeta, K. Simeonidis, M. Angelakeris, E. Natividad, M. Castro, A. Mediano, D.-X. Chen, A. Sanchez, L. Balcells, B. Martínez, Influence of dipolar interactions on hyperthermia properties of ferromagnetic particles, Journal of Applied Physics, 108 (2010) 073918. [303] F. Burrows, C. Parker, R.F.L. Evans, Y. Hancock, O. Hovorka, R.W. Chantrell, Energy losses in interacting fine-particle magnetic composites, Journal of Physics D: Applied Physics, 43 (2010) 474010. [304] T. Jonsson, J. Mattsson, C. Djurberg, F.A. Khan, P. Nordblad, P. Svedlindh, Aging in a Magnetic Particle System, Physical Review Letters, 75 (1995) 4138-4141.

[305] S. Shtrikman, E.P. Wohlfarth, The theory of the Vogel-Fulcher law of spin glasses, Physics Letters A, 85 (1981) 467-470.

[306] J.L. Dormann, D. Fiorani, E. Tronc, On the models for interparticle interactions in nanoparticle assemblies: comparison with experimental results, Journal of Magnetism and Magnetic Materials, 202 (1999) 251-267.

[307] G.T. Landi, The random dipolar-field approximation for systems of interacting magnetic particles, Journal of Applied Physics, 113 (2013) 163908.

[308] R. Hergt, W. Andra, C.G. d'Ambly, I. Hilger, W.A. Kaiser, U. Richter, H.G. Schmidt, Physical limits of hyperthermia using magnetite fine particles, IEEE Transactions on Magnetics, 34 (1998) 3745-3754. [309] A.Z. Wang, V. Bagalkot, C.C. Vasilliou, F. Gu, F. Alexis, L. Zhang, M. Shaikh, K. Yuet, M.J. Cima, R. Langer, P.W. Kantoff, N.H. Bander, S. Jon, O.C. Farokhzad, Superparamagnetic Iron Oxide Nanoparticle-Aptamer Bioconjugates for Combined Prostate Cancer Imaging and Therapy, ChemMedChem, 3 (2008) 1311-1315.

[310] A. Cervadoro, M. Cho, J. Key, C. Cooper, C. Stigliano, S. Aryal, A. Brazdeikis, J.F. Leary, P. Decuzzi, Synthesis of Multifunctional Magnetic NanoFlakes for Magnetic Resonance Imaging, Hyperthermia, and Targeting, ACS Applied Materials \& Interfaces, 6 (2014) 12939-12946.

[311] T. Zargar, A. Kermanpur, Effects of hydrothermal process parameters on the physical, magnetic and thermal properties of $\mathrm{Zn0.3Fe2.704} \mathrm{nanoparticles} \mathrm{for} \mathrm{magnetic} \mathrm{hyperthermia} \mathrm{applications,}$ Ceramics International, 43 (2017) 5794-5804.

[312] C.G. Hadjipanayis, M.J. Bonder, S. Balakrishnan, X. Wang, H. Mao, G.C. Hadjipanayis, Metallic Iron Nanoparticles for MRI Contrast Enhancement and Local Hyperthermia, Small, 4 (2008) 1925-1929.

[313] K.L. Ang, S. Venkatraman, R.V. Ramanujan, Magnetic PNIPA hydrogels for hyperthermia applications in cancer therapy, Materials Science and Engineering: C, 27 (2007) 347-351.

[314] T. Kobayashi, Cancer hyperthermia using magnetic nanoparticles, Biotechnology Journal, 6 (2011) 1342-1347.

[315] D.W. Hwang, H.Y. Ko, J.H. Lee, H. Kang, S.H. Ryu, I.C. Song, D.S. Lee, S. Kim, A Nucleolin-Targeted Multimodal Nanoparticle Imaging Probe for Tracking Cancer Cells Using an Aptamer, Journal of Nuclear Medicine, 51 (2010) 98-105.

[316] J. Weizenecker, B. Gleich, J. Rahmer, H. Dahnke, J. Borgert, Three-dimensional real-time in vivo magnetic particle imaging, Physics in Medicine and Biology, 54 (2009) L1.

[317] L.M. Bauer, S.F. Situ, M.A. Griswold, A.C.S. Samia, High-performance iron oxide nanoparticles for magnetic particle imaging - guided hyperthermia (hMPI), Nanoscale, 8 (2016) 12162-12169.

[318] V. Rieke, K. Butts Pauly, MR thermometry, Journal of Magnetic Resonance Imaging, 27 (2008) 376-390.

[319] M. Huisman, R.M. Staruch, M. Ladouceur-Wodzak, M.A. van den Bosch, D.K. Burns, A. Chhabra, R. Chopra, Non-Invasive Targeted Peripheral Nerve Ablation Using 3D MR Neurography and MRI- 
Guided High-Intensity Focused Ultrasound (MR-HIFU): Pilot Study in a Swine Model, PLOS ONE, 10 (2015) e0144742.

[320] S. Curto, P. Faridi, T.B. Shrestha, M. Pyle, L. Maurmann, D. Troyer, S.H. Bossmann, P. Prakash, An integrated platform for small-animal hyperthermia investigations under ultra-high-field MRI guidance, International Journal of Hyperthermia, 34 (2018) 341-351.

[321] K.K. Vigen, J. Jarrard, V. Rieke, J. Frisoli, B.L. Daniel, K.B. Pauly, In vivo porcine liver radiofrequency ablation with simultaneous MR temperature imaging, Journal of Magnetic Resonance Imaging, 23 (2006) 578-584.

[322] J.A. Moriarty, J.C. Chen, C.M. Purcell, L.C. Ang, R.S. Hinks, R.D. Peters, R.M. Henkelman, D.B. Plewes, M.J. Bronskill, W. Kucharczyk, MRI monitoring of interstitial microwave-induced heating and thermal lesions in rabbit brain in vivo, Journal of Magnetic Resonance Imaging, 8 (1998) 128-135.

[323] T.J.L. Schreurs, R.v. Gorkum, X.U. Zhang, D.J. Faber, T.G.v. Leeuwen, K. Nicolay, G.J. Strijkers, Noninvasive fluence rate mapping in living tissues using magnetic resonance thermometry, Journal of Biomedical Optics, 22 (2017) 1-10, 10.

[324] J. Foiret, K.W. Ferrara, Spatial and Temporal Control of Hyperthermia Using Real Time Ultrasonic Thermal Strain Imaging with Motion Compensation, Phantom Study, PLOS ONE, 10 (2015) e0134938. [325] P. Huang, P. Pande, A. Ahmad, M. Marjanovic, D.R. Spillman, B. Odintsov, S.A. Boppart, Magnetomotive Optical Coherence Elastography for Magnetic Hyperthermia Dosimetry Based on Dynamic Tissue Biomechanics, IEEE Journal of Selected Topics in Quantum Electronics, 22 (2016) 104119.

[326] P.-C. Huang, E.J. Chaney, R.R. Iyer, D.R. Spillman, B. Odintsov, N.A. Sobh, S.A. Boppart, Interstitial magnetic thermotherapy dosimetry based on shear wave magnetomotive optical coherence elastography, Biomed. Opt. Express, 10 (2019) 539-551.

[327] M. Mehrmohammadi, L.L. Ma, Y.-S. Chen, M. Qu, P. Joshi, R.M. Chen, K.P. Johnston, S. Emelianov, Combined photothermal therapy and magneto-motive ultrasound imaging using multifunctional nanoparticles, in: SPIE BiOS, 2010, SPIE, 2010, pp. 8.

[328] C. Jia, S.-W. Huang, Y. Jin, C.H. Seo, L. Huang, J.F. Eary, X. Gao, M. O'Donnell, Integration of photoacoustic, ultrasound, and magnetomotive system, in: SPIE BiOS, 2010, SPIE, 2010, pp. 6.

[329] C.H. Seo, Y. Shi, S.-W. Huang, K. Kim, M. Donnell, Thermal strain imaging: a review, Interface Focus, 1 (2011) 649.

[330] U. Techavipoo, T. Varghese, Q. Chen, T.A. Stiles, J.A. Zagzebski, G.R. Frank, Temperature dependence of ultrasonic propagation speed and attenuation in excised canine liver tissue measured using transmitted and reflected pulses, The Journal of the Acoustical Society of America, 115 (2004) 2859-2865.

[331] R. Maass-Moreno, C.A. Damianou, N.T. Sanghvi, Noninvasive temperature estimation in tissue via ultrasound echo-shifts. Part II. In vitro study, The Journal of the Acoustical Society of America, 100 (1996) 2522-2530.

[332] T. Varghese, J.A. Zagzebski, Q. Chen, U. Techavipoo, G. Frank, C. Johnson, A. Wright, F.T. Lee, Ultrasound monitoring of temperature change during radiofrequency ablation: preliminary in-vivo results, Ultrasound Med. Biol., 28 (2002) 321-329.

[333] Y. Shi, R.S. Witte, S.M. Milas, J.H. Neiss, X.C. Chen, C.A. Cain, M.O. Donnell, Ultrasonic thermal imaging of microwave absorption, in: IEEE Symposium on Ultrasonics, 2003, 2003, pp. 224-227 Vol.221.

[334] J. Shah, S. Park, S. Aglyamov, T. Larson, L. Ma, K. Sokolov, K. Johnston, T. Milner, S.Y. Emelianov, Photoacoustic imaging and temperature measurement for photothermal cancer therapy, Journal of Biomedical Optics, 13 (2008) 034024-034024-034029.

[335] M. Harada, On the magnetic field of a solenoid, American Journal of Physics, 54 (1986) 10651065.

[336] M. Subramanian, A. Miaskowski, A.K. Mahapatro, J. Dobson, Practical bioinstrumentation developments for AC magnetic field-mediated magnetic nanoparticle heating applications, Applied Physics A, 125 (2019) 194. 
[337] A.T. Barker, An introduction to the basic principles of magnetic nerve stimulation, Journal of clinical neurophysiology: official publication of the American Electroencephalographic Society, 8 (1991) 26-37.

[338] A.V. Peterchev, R. Jalinous, S.H. Lisanby, A Transcranial Magnetic Stimulator Inducing NearRectangular Pulses With Controllable Pulse Width (cTMS), IEEE Transactions on Biomedical Engineering, 55 (2008) 257-266.

[339] O. Bonnefous, P. Pesqué, Time domain formulation of pulse-Doppler ultrasound and blood velocity estimation by cross correlation, UltIm, 8 (1986) 73-85.

[340] R. Boucher, J. Hassab, Analysis of discrete implementation of generalized cross correlator, IEEE Transactions on Acoustics, Speech, and Signal Processing, 29 (1981) 609-611.

[341] F.A. Duck, Physical Properties of Tissue: A Comprehensive Reference Book, Academic Press, 1990.

[342] N.R. Miller, J.C. Bamber, P.M. Meaney, Fundamental limitations of noninvasive temperature imaging by means of ultrasound echo strain estimation, Ultrasound Med. Biol., 28 (2002) 1319-1333. [343] C. Simon, P. VanBaren, E.S. Ebbini, Two-dimensional temperature estimation using diagnostic ultrasound, IEEE Transactions on Ultrasonics, Ferroelectrics, and Frequency Control, 45 (1998) 10881099.

[344] F. Kallel, J. Ophir, A Least-Squares Strain Estimator for Elastography, UltIm, 19 (1997) 195-208. [345] M. Mehrmohammadi, J. Oh, L. Ma, E. Yantsen, T. Larson, S. Mallidi, S. Park, K.P. Johnston, K. Sokolov, T. Miner, S. Emelianov, 8B-2 Imaging of Iron Oxide Nanoparticles Using Magneto-Motive Ultrasound, in: Ultrasonics Symposium, 2007. IEEE, 2007, pp. 652-655.

[346] M. Pernot, M. Tanter, J. Bercoff, K.R. Waters, M. Fink, Temperature estimation using ultrasonic spatial compound imaging, IEEE Transactions on Ultrasonics, Ferroelectrics, and Frequency Control, 51 (2004) 606-615.

[347] P. Faridi, S.H. Bossmann, P. Prakash, CHAPTER 8 Image-Guided Cancer Thermal Therapies, in: Magnetic Nanomaterials: Applications in Catalysis and Life Sciences, The Royal Society of Chemistry, 2017, pp. 195-220.

[348] H. Dähring, J. Grandke, U. Teichgräber, I. Hilger, Improved Hyperthermia Treatment of Tumors Under Consideration of Magnetic Nanoparticle Distribution Using Micro-CT Imaging, Mol. Imag. Biol., 17 (2015) 763-769.

[349] K. Hayashi, M. Nakamura, W. Sakamoto, T. Yogo, H. Miki, S. Ozaki, M. Abe, T. Matsumoto, K. Ishimura, Superparamagnetic nanoparticle clusters for cancer theranostics combining magnetic resonance imaging and hyperthermia treatment, Theranostics, 3 (2013) 366.

[350] H.F. Rodrigues, F.M. Mello, L.C. Branquinho, N. Zufelato, E.P. Silveira-Lacerda, A.F. Bakuzis, Realtime infrared thermography detection of magnetic nanoparticle hyperthermia in a murine model under a non-uniform field configuration, International Journal of Hyperthermia, 29 (2013) 752-767.

[351] J. Kolosnjaj-Tabi, R. Di Corato, L. Lartigue, I. Marangon, P. Guardia, A.K.A. Silva, N. Luciani, O. Clément, P. Flaud, J.V. Singh, P. Decuzzi, T. Pellegrino, C. Wilhelm, F. Gazeau, Heat-Generating Iron Oxide Nanocubes: Subtle "Destructurators" of the Tumoral Microenvironment, ACS Nano, 8 (2014) 4268-4283.

[352] F.R. Harley, C. Gustavo, M.M. Francyelli, Z. Nicholas, S.-L. Elisângela, F.B. Andris, Precise determination of the heat delivery during in vivo magnetic nanoparticle hyperthermia with infrared thermography, Physics in Medicine \& Biology, 62 (2017) 4062.

[353] B. Mehdaoui, J. Carrey, M. Stadler, A. Cornejo, C. Nayral, F. Delpech, B. Chaudret, M. Respaud, Influence of a transverse static magnetic field on the magnetic hyperthermia properties and highfrequency hysteresis loops of ferromagnetic FeCo nanoparticles, Applied Physics Letters, 100 (2012) 052403.

[354] K. Murase, H. Takata, Y. Takeuchi, S. Saito, Control of the temperature rise in magnetic hyperthermia with use of an external static magnetic field, Physica Medica: European Journal of Medical Physics, 29 (2013) 624-630. 
[355] C. Lai, D.E. Kruse, C.F. Caskey, D.N. Stephens, P.L. Sutcliffe, K.W. Ferrara, Noninvasive thermometry assisted by a dual-function ultrasound transducer for mild hyperthermia, IEEE Transactions on Ultrasonics, Ferroelectrics, and Frequency Control, 57 (2010) 2671-2684.

[356] J. Seo, S.K. Kim, Y. Kim, K. Choi, D.G. Kong, W. Bang, Motion Compensation for Ultrasound Thermal Imaging Using Motion-Mapped Reference Model: Anin vivoMouse Study, IEEE Transactions on Biomedical Engineering, 61 (2014) 2669-2678.

[357] D. Liu, J. Jiang, J. Bischof, J. Ballard, E. Ebbini, Real-time monitoring of thermal and mechanical response to sub-therapeutic HIFU beams in vivo, in: 2010 IEEE International Ultrasonics Symposium, 2010, pp. 2254-2257.

[358] M. Johannsen, U. Gneveckow, B. Thiesen, K. Taymoorian, C.H. Cho, N. Waldöfner, R. Scholz, A. Jordan, S.A. Loening, P. Wust, Thermotherapy of Prostate Cancer Using Magnetic Nanoparticles: Feasibility, Imaging, and Three-Dimensional Temperature Distribution, Eur. Urol., 52 (2007) 16531662.

[359] M. Johannsen, B. Thiesen, P. Wust, A. Jordan, Magnetic nanoparticle hyperthermia for prostate cancer, International Journal of Hyperthermia, 26 (2010) 790-795.

[360] O. Grauer, M. Jaber, K. Hess, M. Weckesser, W. Schwindt, S. Maring, J. Wölfer, W. Stummer, Combined intracavitary thermotherapy with iron oxide nanoparticles and radiotherapy as local treatment modality in recurrent glioblastoma patients, Journal of Neuro-Oncology, 141 (2019) 83-94. 